\title{
RESOLVENT NEAR ZERO ENERGY ON RIEMANNIAN SCATTERING (ASYMPTOTICALLY CONIC) SPACES
}

\author{
ANDRÁS VASY
}

\begin{abstract}
We give resolvent estimates near zero energy on Riemannian scattering, i.e. asymptotically conic, spaces, and their generalizations, using a uniform microlocal Fredholm analysis framework.
\end{abstract}

\section{INTRODUCTION AND RESULTS}

In this paper we consider geometric generalizations of Euclidean low energy resolvent estimates, such as for the resolvent of the Euclidean Laplacian plus a decaying potential, in a Fredholm framework. Such an analysis is relevant for instance for the asymptotic behavior of the solutions of the wave equation, though this connection will be pursued elsewhere as it requires some additional ingredients. Indeed, one motivation for the present paper is understanding waves on Kerr spacetimes. However, another motivation for the present work is even just the description of Euclidean phenomena, namely what is really happening for low energies. In one sense this has been addressed by Guillarmou and Hassell in a series of works [17, 18, via constructing a parametrix for the resolvent family; here we proceed by directly obtaining Fredholm estimates, which are actually less technically involved, and also are not straightforward to read off from the parametrix result, especially as we need to work on variable order, or anisotropic, Sobolev spaces. A forthcoming companion paper will give a slightly different perspective that is even more amenable towards the study of wave propagation, though somewhat less so for direct spectral theory applications.

Let us start by recalling the Euclidean results. For this purpose, we initially let $g_{0}$ be the Euclidean metric, $g$ metric on $\mathbb{R}^{n}$ with $g-g_{0} \in S^{-\delta}, \delta>0$ (i.e. $\left.g_{i j}-\left(g_{0}\right)_{i j} \in S^{-\delta}\right), g$ positive definite, $V \in S^{-\delta}$, real. Recall here that $S^{m}\left(\mathbb{R}_{z}^{n}\right)$ is the space of symbols of order $m$ : for all $\alpha$

$$
\left|D_{z}^{\alpha} a(z)\right| \leq C_{\alpha}\langle z\rangle^{m-|\alpha|} ; \quad\langle z\rangle=\left(1+|z|^{2}\right)^{1 / 2} .
$$

In order to make a connection with the upcoming generalization, we also give these estimates in a different form (away from origin): for all $k$ and sequences $i_{1}, \ldots, i_{k}$, $j_{1}, \ldots, j_{k}$,

$$
\left|W_{1} \ldots W_{k} a(z)\right| \leq C_{k}\langle z\rangle^{m}
$$

Date: August 18, 2018.

2000 Mathematics Subject Classification. Primary 58J50; Secondary 58J40, 35P25, 58J47.

The author gratefully acknowledges partial support from the NSF under grant numbers DMS1361432 and DMS-1664683 and from a Simons Fellowship. 
where $W_{r}=z_{i_{r}} D_{z_{j_{r}}}$; of course, localized to the region where $\left|z_{n}\right|>c\left|z_{j}\right|$ for $j \neq n$ $(c>0)$ and $|z|>1$, it suffices to consider $W_{r}=z_{n} D_{z_{j_{r}}}$. We note that $V$ nonreal, but $\operatorname{Im} V \in S^{-1-\delta}$ (so a slightly stronger constraint), would only cause some possible finite rank issues below.

Under these assumptions

$$
H=\Delta_{g}+V
$$

is self-adjoint on $L^{2}\left(\mathbb{R}^{n}\right)$, so $H-\lambda, \lambda \in \mathbb{C} \backslash \mathbb{R}$ is invertible, e.g. as a map

$$
H-\lambda: H^{s+2, l} \rightarrow H^{s, l}, s, l \in \mathbb{R} ;
$$

standard elliptic theory implies that the inverse is independent of the particular space chosen for the inversion (in the sense that e.g. the restriction of the inverse to the dense subspace given by Schwartz functions is the same). Moreover, the spectrum in $(-\infty, 0)$ is discrete, with 0 a possible accumulation point (this happens e.g. for negative Coulomb-like potentials, which are Coulomb-like in terms of decay at infinity); $[0, \infty)$ the essential spectrum. Here $H^{s, l}=\langle z\rangle^{-l} H^{s}$, where $H^{s}$ is the standard Sobolev space.

While $H-\lambda$ will no longer be invertible between the weighted Sobolev spaces when $\lambda>0$, the limiting absorption principle states that

$$
(H-(\lambda \pm i 0))^{-1}=\lim _{\epsilon \rightarrow 0}(H-(\lambda \pm i \epsilon))^{-1}
$$

exist e.g. as strong limits (indeed, norm limits) in $\mathcal{L}\left(H^{s, l}, H^{s+2, l^{\prime}}\right), l>\frac{1}{2}, l^{\prime}<-\frac{1}{2}$ (so $l-l^{\prime}>1$ ). Under stronger assumptions (and $n \geq 3$, which will be assumed from now), which are necessary in view of Coulomb-like potentials, $V \in S^{-2-\delta}, \delta>0$, 0 is not an accumulation point of the spectrum, and under stronger restrictions on $l, l^{\prime}$, in particular $l-l^{\prime}>2,(H-(\lambda \pm i 0))^{-1}$ is uniformly bounded between the weighted Sobolev spaces as $\lambda \rightarrow 0$ if there are no 0 -energy bound states $\left(L^{2}\right.$ nullspace of $H$ ) or half-bound states; the latter are elements of the nullspace of $H$ on a larger space of distributions that will be discussed later, see the discussion around (1.3). Such results go back to Jensen and Kato [26]; the stated results are from recent works of Bony and Häfner [5], though potentials are not considered there, just divergence form second order operators, and Rodnianski and Tao [33] who do consider such potentials and indeed more generally asymptotically conic manifolds. We also refer to the work of Müller and Strohmaier [30] for a discussion of the analytic continuation, near zero energy, of the resolvent of the Laplacian on spatially compact perturbations of cones using the theory of Hahn meromorphic functions they develop.

We remark here that there are also high energy estimates under assumptions on the geodesic flow, with the best case scenario if all geodesics are backward and forward non-trapped, i.e. tend to infinity in both directions, see [32, 16, 15, 46], and also [43] for a general asymptotically conic result.

It is natural to ask what kind of structure of Euclidean space is involved in these results. One way to address this is via constructing geometric generalizations. Another natural question is whether one can make the function spaces more precise. For instance, can one fit these estimates into a Fredholm (here typically invertible) statement? Such frameworks are necessarily sharp in a sense: one can only significantly (in not an essentially finite dimensional way) change the domain if one changes the target space and vice versa. 
Note that the estimates above are lossy: the actual phenomenon is a version of real principal type propagation, with radial points (discussed below), here in terms of decay, so the difference between the two decay indices should be 1 for the positive energy estimates (i.e we have a loss of $\epsilon>0$ ) - it is the radial points that prevent the optimal choice if one works with constant powers of $\langle z\rangle$ as the weights.

In this paper we address these questions. A natural geometric generalization is asymptotically conic spaces. A conic metric, with cross section a Riemannian manifold $(Y, h)$, is the metric $g_{0}=d r^{2}+r^{2} h$ on $\mathbb{R}_{r}^{+} \times Y$; Euclidean space is a cone over the sphere. In local coordinates, such a metric is a linear combination, with $\mathcal{C}^{\infty}(Y)$-coefficients, of $d r$ and $r d y_{j}, y_{j}$ local coordinates on $Y$, with some restrictions.

We want to generalize these coefficients to relax the warped product structure of the conic metric. Identifying a coordinate chart in $Y$ with a coordinate chart on the sphere $\mathbb{S}^{n-1}$, we are working in an open conic subset $O$ of $\mathbb{R}^{n}$ near infinity. (So this is a natural generalization of the process of going from $\mathbb{R}^{n}$ to say compact manifolds without boundary.) From this perspective, below we replace the coefficients $\mathcal{C}^{\infty}(Y)$ by a symbolic statement, namely coefficients in $S^{0}(O)$, differing from elements of $\mathcal{C}^{\infty}(Y)$ by $S^{-\delta}(O), \delta>0$; this parallels the setting of Rodnianski and Tao 33.

Let us rephrase this in a compactified notation introduced by Melrose [27, where 'scattering', or sc-structures were discussed. So let $x=\frac{1}{r}$, and add $x=0$ as an ideal boundary at infinity, i.e. work with $[0, \infty)_{x} \times Y$. The metrics considered above are then a linear combination of symmetric tensors formed from $\frac{d x}{x^{2}}$ and $\frac{d y_{j}}{x}$. Then being an element of $\mathcal{C}^{\infty}\left([0, \infty)_{x} \times Y\right)$ near the ideal boundary means exactly being a classical symbol of order 0 (on $\mathbb{R}^{n}$ or under the local conic identification), i.e. having an asymptotic expansion in non-positive integer powers of $r$ (asymptotic in the symbolic sense), with the expansion being just Taylor series at the boundary, i.e. an asymptotic expansion in non-negative integer powers of $x$. A straightforward computation shows that if $Y$ is the sphere, i.e. we are discussing $\mathbb{R}^{n} \backslash\{0\}$, with an ideal boundary at infinity added, then linear combinations of $\frac{d x}{x^{2}}$ and $\frac{d y_{j}}{x}$ with $\mathcal{C}^{\infty}\left([0, \infty)_{x} \times Y\right)$, resp. symbolic (on the complement of the origin), coefficients, are exactly the same as linear combinations of the coordinate 1 -forms $d z_{j}$ with the same kind of coefficients; this property persists for general $Y$ locally on open subsets $O$ of the kind considered above.

In Melrose's notation, if $X$ is a manifold with boundary which near $\partial X$ is of the form $\left[0, x_{0}\right)_{x} \times Y$, one would say that a metric with such coefficients, namely an sc-metric, is a $\mathcal{C}^{\infty}$ section of ${ }^{\mathrm{sc}} T^{*} X \otimes_{s}{ }^{\mathrm{sc}} T^{*} X$, with ${ }^{\mathrm{sc}} T^{*} X$ being locally spanned by $\frac{d x}{x^{2}}$ and $\frac{d y_{j}}{x}$. (See Section 5 for additional discussion.) In general $a \in S^{m}$ becomes $\left(x \partial_{x}\right)^{\alpha} \partial_{y}^{\beta} a \in x^{-m} L^{\infty}$ from this perspective (cf. the earlier linear vector field characterization); more invariantly this can be replaced by $W_{1} \ldots W_{k} a \in x^{-m} L^{\infty}$ for all $k$ and $W_{j} \in \mathcal{V}_{\mathrm{b}}(X)$, where $\mathcal{V}_{\mathrm{b}}(X)$ is the set of all smooth vector fields tangent to $\partial X$. (Thus, in the non-compactified notation, locally and near $\partial X$, i.e. in an asymptotically conic set near infinity in $\mathbb{R}_{z}^{n}$, a spanning set for $\mathcal{V}_{\mathrm{b}}(X)$ is given by linear vector fields $z_{i} D_{z_{j}}, i, j=1, \ldots, n$; in the region where $\left|z_{i}\right|<C\left|z_{n}\right|,|z|>1$, this means that one can use the local basis $z_{n} D_{z_{j}}, j=1, \ldots, n$, again with classical symbols of order 0 , cf. above, as coefficients.) A symbolic section of ${ }^{{ }^{s c}} T^{*} X^{\otimes_{s}^{2}}$ is denoted by $S^{m}\left(X,{ }^{\text {sc }} T^{*} X^{\otimes_{s}^{2}}\right)$.

So in general let $X$ be an $n$-dimensional manifold with boundary, $n \geq 3$, with $X^{\circ}$ equipped with a metric $g$ which is asymptotically conic (near $\partial X$ ) in the following 
precise sense. We consider $X$ equipped with a sc-metric $g$, i.e. a symbolic of order 0 section of ${ }^{\mathrm{sc}} T^{*} X \otimes_{s}{ }^{\mathrm{sc}} T^{*} X$ which at $\partial X$ (i.e. 'metric infinity') is conic:

$$
g-g_{0} \in S^{-\delta}\left(X ;{ }^{\mathrm{sc}} T^{*} X\right), g_{0}=x^{-4} d x^{2}+x^{-2} g_{\partial X}, \delta>0,
$$

$g_{\partial X}$ a metric on $\partial X$, i.e. $g$ is asymptotic to a conic metric $g_{0}$ on $(0, \infty) \times \partial X$. A special case of the operators we then consider is the spectral family of the Laplacian plus a decaying potential,

$$
P(\sigma)=\Delta_{g}+V-\sigma^{2}
$$

where $V \in S^{-2-\delta}(X)$, and we are interested in the $\sigma \rightarrow 0$ limit. (For $\sigma$ bounded away from $0, V \in S^{-\delta}(X)$ real-valued suffices.)

Thus, $P(\sigma)$ is a family of (conormal/symbolic) scattering differential operators (elements of $S^{0} \operatorname{Diff}_{\mathrm{sc}}(X)$ ), i.e. is a finite sum of products (possibly empty) of scattering vector fields $\mathcal{V}_{\mathrm{sc}}(X)=x \mathcal{V}_{\mathrm{b}}(X)$, with local basis $x^{2} D_{x}, x D_{y_{j}}$, dual to the scattering covectors discussed above, with symbolic of order 0 coefficients. (See Section 5 for more detail.) In a non-compactified notation, over conic subsets of $\mathbb{R}_{z}^{n}$, this means that it is a linear combination of $D_{z}^{\alpha}$ with coefficients which are symbols of order 0 . In fact, modulo $S^{-\delta}$ the coefficients are equal to a classical symbol. (This holds even for $V \in S^{-\delta}(X)$.) (The notation Diff ${ }_{\mathrm{sc}}(X)$ is reserved for $\mathcal{C}^{\infty}(X)$, i.e. classical symbolic of order 0 , coefficients.) This scattering structure is what is required for the non-zero $\sigma$ limiting absorption principle considerations, which is the reason $V \in S^{-\delta}(X)$ is acceptable for that purpose.

However, our $P(\sigma)$ has a stronger structure. Recall that $\operatorname{Diff}_{\mathrm{b}}(X)$ is the differential operator algebra generated by $\mathcal{V}_{\mathrm{b}}(X)$ with $\mathcal{C}^{\infty}(X)$ coefficients; $S^{m} \operatorname{Diff}_{\mathrm{b}}(X)$ means that the coefficients are symbols of order $m$. Then

$$
\Delta_{g} \in x^{2} S^{0} \operatorname{Diff}_{\mathrm{b}}^{2}(X)=S^{-2} \operatorname{Diff}_{\mathrm{b}}^{2}(X), V \in S^{-2-\delta} \operatorname{Diff}_{\mathrm{b}}^{0}(X),
$$

so

$$
P(\sigma)+\sigma^{2} \in S^{-2} \operatorname{Diff}_{\mathrm{b}}^{2}(X),
$$

with a classical symbolic leading term. This stronger structure plays a key role in the zero energy limit considered in this paper.

More generally, though the results are interesting even without this extension, with $\delta>0$, we consider families of the form

$$
P(\sigma)=P(0)+\sigma Q-\sigma^{2}, \quad P(0) \in x^{2} \operatorname{Diff}_{\mathrm{b}}^{2}(X), Q \in S^{-2-\delta} \operatorname{Diff}_{\mathrm{b}}^{1}(X)
$$

and $P(0)$ elliptic, $P(\sigma)$ is symmetric for $\sigma$ real, and $P(0)-\Delta_{g} \in S^{-2-\delta} \operatorname{Diff}_{\mathrm{b}}^{2}(X)$, with the b-differential operators discussed below. Such a generalization is useful for Kerr-type spaces, and it does not affect any arguments. For actual Kerr spacetimes, near the event horizon we lose ellipticity as well, but it is straightforward to modify arguments using the Kerr-de Sitter results from [40, and we do that here in the penultimate Section 6. (We explicitly discuss the asymptotic form of the Kerr wave operator in that section.) Indeed, $Q$ can be replaced by any family of operators $Q(\sigma)$ of the same class, depending smoothly on $\sigma$, for all considerations below. Note that there is extensive literature on related aspects of the Kerr setting, including [8, 9, 34, 7, 1, 25, 37, 38, and many other papers, most of which is for the actual spacetimes, rather than the Fourier transformed problem; here our interest is in a robust treatment of a general class of such operators, so we restrict to brief remarks for the changes necessary in Section 6, and we refer to the introduction of 22 for a more extensive discussion. 


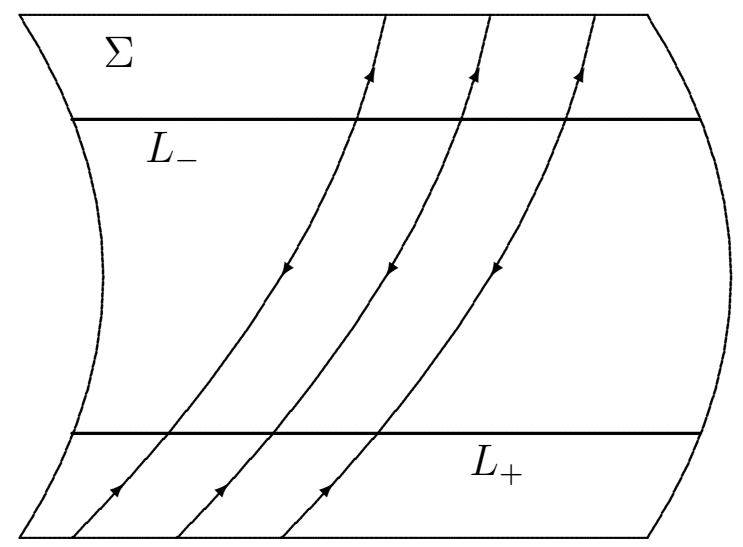

Figure 1. The characteristic set $\Sigma$, here a $\mathbb{T}^{2}$ (with opposite sides on the figure identified), and source/sink manifolds $L_{-}$, resp. $L_{+}$, of the Hamilton flow.

We are then interested in limiting absorption resolvent estimates along the spectrum (it is straightforward to add an imaginary part of the correct sign, corresponding to $\pm i 0$ ) as the spectral parameter $\sigma$ tends to 0 . We first recall the basic limiting absorption principle, for $\sigma \neq 0$, proved in this geometric setting by Melrose [27, generalizing (1.1), but in a different, Fredholm setting, in which it was shown in [45, Section 5.4.8], see also [44, Section 4]. This setting involves variable order (or anisotropic) scattering Sobolev spaces; such spaces in the standard microlocal setting have been used by Unterberger [39] and Duistermaat [11, but here it is the weight, i.e. the decay order that is variable, corresponding to the operator $P(\sigma)$ being elliptic in the standard, differential order, sense, thus the differential order of the Sobolev space playing no role. (We recall that such variable order Sobolev spaces played a key role in recent advances in dynamical systems, see e.g. [14, 13.) Via the identification of open subsets of $X$ near $\partial X$ with asymptotically conic subsets of $\mathbb{R}^{n}$, such spaces (locally) reduce to variable order Sobolev spaces on $\mathbb{R}^{n}$, where again it is the decay order that varies. (Via the Fourier transform, however, microlocally these can be reduced to spaces with variable differential order!) These Sobolev spaces are defined using pseudodifferential operators of variable order. Now, in regions where the operator is microlocally elliptic, the order of the Sobolev space is immaterial (all orders work equally well for Fredholm theory); only at the characteristic set, which in this case is $G-\sigma^{2}=0, G$ the dual metric function on ${ }^{\mathrm{sc}} T^{*} X$, is it relevant. Within the characteristic set, the Hamilton flow tends to, i.e. the $H_{G}$ integral curves tend to, sink/source manifolds $\left(L_{-}\right.$, resp. $L_{+}$, see Figure 1), called radial sets, in the forward/backward direction. The variable order is a function $r$ that needs to be monotone along the $H_{G}$-flow (corresponding to propagation of singularities, or rather of regularity), and it needs to be greater than, resp. less than, a threshold value, here $-1 / 2$, at one, resp. the other, radial set. The choice is thus whether the decay order is $>-1 / 2$ at the source manifold or the sink manifold; these two possibilities correspond to the incoming/outgoing limiting resolvents. The Fredholm statement is that for any $s$ and for variable order 
$r$ satisfying these requirements

$$
P(\sigma):\left\{u \in H_{\mathrm{sc}}^{s+2, r}: P(\sigma) u \in H_{\mathrm{sc}}^{s, r+1}\right\} \rightarrow H_{\mathrm{sc}}^{s, r+1}
$$

is Fredholm, indeed in this case invertible. Notice that the loss of $\epsilon>0$ in the usual way the limiting absorption principle is stated, cf. (1.1), disappears with these variable order spaces ( $r+1$ vs. $r$, exactly the real principal type numerology). (We remark that, with essentially the same proof, one also has lossless high energy estimates on large parameter, or semiclassical, scattering Sobolev spaces.)

Concretely, with $\tau, \mu$ the fiber coordinates, in the sc-notation, on ${ }^{{ }^{c}} T^{*} X$, i.e. writing covectors as $\tau \frac{d x}{x^{2}}+\mu \cdot \frac{d y}{x}$, one can take for $\beta>0$

$$
r=-\frac{1}{2} \pm \beta \frac{\tau}{\sqrt{\tau^{2}+|\mu|_{g_{\partial X}}^{2}}},
$$

which in the Euclidean case just means

$$
r=-\frac{1}{2} \mp \beta \frac{z \cdot \zeta}{|z||\zeta|}
$$

as the variable order.

These Fredholm estimates, i.e. the propagation of singularities estimates, including radial points, are proved by positive commutator estimates, which become degenerate (quadratic vanishing of symbol) at 0 energy. However, it turns out that phrasing these estimates as b-estimates one can make them uniform as $\sigma \rightarrow 0$.

We already introduced the b-differential operators, $\operatorname{Diff}_{\mathrm{b}}(X)$; there is a pseudodifferential algebra $\Psi_{\mathrm{b}}(X)$ microlocalizing it, originating in Melrose's work, see 29] for a detailed treatment, and recalled in Section 2. The b-Sobolev spaces are then based on these operators; recalling that the symbolic estimates amount to iterative regularity with respect to elements of $\mathcal{V}_{\mathrm{b}}(X)$ relative to $L^{\infty}$, we see that these Sobolev spaces are thus essentially a finite regularity version of $L^{2}$-based (rather than $L^{\infty}$-based) symbol classes. As an aside, b-structures are typically associated with 'cylindrical ends' in the Riemannian geometry literature, but as we discussed $P(0)$ can be considered as $x^{2}$ times an unweighted second order b-differential operator, i.e. 'conformally unweighted b'.

Let $H_{\mathrm{b}}^{\tilde{r}, l}$ be the b-Sobolev space of differential order $\tilde{r}$, considered as a function on ${ }^{\mathrm{b}} S^{*} X=\left({ }^{\mathrm{b}} T^{*} X \backslash o_{\mathrm{b}}\right) / \mathbb{R}^{+}$(equivalently, a homogeneous degree zero function on ${ }^{\mathrm{b}} T^{*} X \backslash o_{\mathrm{b}}, o_{\mathrm{b}}$ the zero section), and weight $l$, relative to the scattering $L^{2}$-space $L^{2}=L_{\mathrm{sc}}^{2}$; this is a notion we recall and explain presently. Thus, in this b-setting the differential order, not the decay order, is variable, unlike in the sc-setting. If $\tilde{r}$ is a positive integer $m$, then

$$
H_{\mathrm{b}}^{\tilde{r}, l}=\left\{u \in H_{\mathrm{b}}^{0, l}=x^{l} L^{2}: k \leq m, W_{1}, \ldots, W_{k} \in \mathcal{V}_{\mathrm{b}}(X) \Rightarrow W_{1} \ldots W_{k} u \in x^{l} L^{2}\right\} .
$$

In local coordinates, the regularity statement is simply that

$$
j+|\alpha| \leq m \Rightarrow\left(x D_{x}\right)^{j} D_{y}^{\alpha} u \in x^{l} L^{2} .
$$

Notice that, for suitable values of $\tilde{r}$ (constant) and $l$, the $j=m$ control, i.e. $\left(x D_{x}\right)^{m} u \in x^{l} L^{2}$, can be used to estimate $u$ in $x^{l} L^{2}$ by Hardy-type inequalities, so in some cases these b-Sobolev spaces are, in case of compactified $\mathbb{R}^{n}$, the usual (weighted) homogeneous Sobolev spaces. For negative integers $\tilde{r}$, the spaces can be defined either via duality or using elliptic b-pseudodifferential operators. In general, for a function $\tilde{r}$, the Sobolev space $H_{\mathrm{b}}^{\tilde{r}, l}$ is defined by taking $-N<\inf \tilde{r}$, and 
$\Lambda \in \Psi_{\mathrm{b}}^{\tilde{r}, l}$ elliptic, e.g. with principal symbol $x^{-l}\left(\tau_{\mathrm{b}}^{2}+\left|\mu_{\mathrm{b}}\right|^{2}\right)^{\tilde{r} / 2},\left|\mu_{\mathrm{b}}\right|$ e.g. the length with respect to $g_{\partial X}$ (but all choices are equivalent),

$$
H_{\mathrm{b}}^{\tilde{r}, l}=\left\{u \in H_{\mathrm{b}}^{-N, l}: \Lambda u \in L^{2}\right\} .
$$

Melrose [29, see also Guillarmou and Hassell [17, Theorem 2.1] for explicitly this statement when $\tilde{r}$ is constant, showed that

$$
P(0): H_{\mathrm{b}}^{\tilde{r}, l} \rightarrow H_{\mathrm{b}}^{\tilde{r}-2, l+2}
$$

is Fredholm of index 0 when $|l+1|<\frac{n-2}{2}$; cf. Section 5.6 of [45] for a general $\tilde{r}$ statement, and see the comments below. Note that $l=-1$ corresponds to the weights of the domain and target spaces being symmetric relative to $L^{2}$ (in the sense of being $L^{2}$-duals of each other, considering just these weights, not the differential orders). By the ellipticity of $P(0), \tilde{r}$ is actually irrelevant for the nullspace considerations (i.e. the nullspace is independent of this choice), and, by indicial root considerations, in the range $|l+1|<\frac{n-2}{2}$ invertibility is also independent of $l$, i.e. when $P(0)$ is considered as such an operator, for all $\epsilon>0$,

$$
\operatorname{Ker} P(0) \subset H_{\mathrm{b}}^{\infty,-1+\frac{n-2}{2}-\epsilon}=H_{\mathrm{b}}^{\infty, \frac{n-4}{2}-\epsilon} .
$$

Note that when $n \leq 4$, this Fredholm, of index 0 , range excludes $l=0$, thus the choice of the standard $L^{2}$ space, so for our purposes it is not just the $L^{2}$ nullspace that matters, rather the nullspace on a bigger function space, $H_{\mathrm{b}}^{\infty, \frac{n-4}{2}-\epsilon}$, where $\epsilon>0$ can be taken arbitrarily small (and should be $<n-2$ ). Non- $L^{2}$ elements of this nullspace, if they exist, are called half bound states; $L^{2}$ elements are called bound states. On the other hand, for $n>4$, necessarily any such zero eigenvalue is an $L^{2}$-eigenvalue. Also note that if $P(0)=\Delta_{g}$, then the maximum principle implies that the nullspace is trivial for all $n \geq 3$ since any element $u$ of this nullspace is in a weighted, infinite order differentiability, b-Sobolev space that is decaying relative to $L_{\mathrm{b}}^{2}$ (as $L^{2}=x^{n / 2} L_{\mathrm{b}}^{2}, L_{\mathrm{b}}^{2}$ being the $L^{2}$ space relative to a $b$-density, e.g. in local coordinates $\frac{d x}{x} d y$, and we have membership of $u$ in $x^{(n-4) / 2-\epsilon} L^{2}$ for all $\left.\epsilon>0\right)$, and thus decaying at infinity in the sup sense by Sobolev embedding. (There are also direct $L^{2}$-based arguments available using integration by parts, which needs to be justified, but which can be done.)

In order to proceed, write the b-dual variable of $x$ as $\tau_{\mathrm{b}}$, that of $y_{j}$ as $\left(\mu_{\mathrm{b}}\right)_{j}$, so covectors are written as

$$
\tau_{\mathrm{b}} \frac{d x}{x}+\sum_{j}\left(\mu_{\mathrm{b}}\right)_{j} d y_{j}
$$

Then a typical symbol of a b-pseudodifferential operator of order $\tilde{r}$, where $\tilde{r}$ may be a function on ${ }^{\mathrm{b}} S^{*} X$, is $\left(\tau_{\mathrm{b}}^{2}+\left|\mu_{\mathrm{b}}\right|^{2}\right)^{\tilde{r} / 2}$, with $\left|\mu_{\mathrm{b}}\right|$ being the length with respect to $g_{\partial X}$. A typical example of a variable differential order that we consider is

$$
\tilde{r}=\frac{1}{2}-(l+1) \pm \beta \frac{\tau_{\mathrm{b}}}{\left(\tau_{\mathrm{b}}^{2}+\left|\mu_{\mathrm{b}}\right|^{2}\right)^{1 / 2}},
$$

where $\beta>0$, and \pm gives rather different function spaces that will correspond to the $\pm i 0$ limit of the resolvent.

We now explain the connection to the scattering spaces. The symbol of a representative (for our purposes) elliptic b-pseudodifferential operator in $\Psi_{\mathrm{b}}^{\tilde{r}, l}$, which 
$\operatorname{maps} H_{\mathrm{b}}^{\tilde{r}, l}$ to $L^{2}$, is $x^{-l}\left(\tau_{\mathrm{b}}^{2}+\left|\mu_{\mathrm{b}}\right|^{2}\right)^{\tilde{r} / 2}$. Now,

$$
\tau_{\mathrm{b}} \frac{d x}{x}+\sum_{j}\left(\mu_{\mathrm{b}}\right)_{j} d y_{j}=\left(x \tau_{\mathrm{b}}\right) \frac{d x}{x^{2}}+\sum_{j}\left(x \mu_{\mathrm{b}}\right)_{j} \frac{d y_{j}}{x},
$$

i.e. the ${ }^{\mathrm{sc}} T^{*} X$ fiber coordinates are $\tau=x \tau_{\mathrm{b}}, \mu=x \mu_{\mathrm{b}}$, shows that in terms of the scattering cotangent bundle coordinates, this symbol is

$$
x^{-l}\left(\tau_{\mathrm{b}}^{2}+\left|\mu_{\mathrm{b}}\right|^{2}\right)^{\tilde{r} / 2}=x^{-l-\tilde{r}}\left(\tau^{2}+|\mu|^{2}\right)^{\tilde{r} / 2},
$$

which is, away from the 0 -section (where it is singular) the symbol of an elliptic scdifferential operator in $\Psi_{\mathrm{sc}}^{\tilde{r}, l+\tilde{r}}$. Recalling that, due to ellipticity, the sc-differential order is irrelevant for the limiting absorption principle, we see that microlocally near the sc-characteristic set, for $\sigma \neq 0$ (so the characteristic set is away from the zero section), these Sobolev spaces correspond to $H_{\mathrm{sc}}^{*, r}$,

$$
r=l+\tilde{r}=-\frac{1}{2} \pm \beta \frac{\tau}{\left(\tau^{2}+|\mu|^{2}\right)^{1 / 2}},
$$

in agreement with the orders that appeared in the limiting absorption principle above!

Now, for $\sigma \neq 0, P(\sigma)$ does not map $H_{\mathrm{b}}^{\tilde{r}, l} \rightarrow H_{\mathrm{b}}^{\tilde{r}-2, l+2}$. We shall instead consider $P(\sigma)$ as a map

$$
\left\{u \in H_{\mathrm{b}}^{\tilde{r}, l}: P(\sigma) u \in H_{\mathrm{b}}^{\tilde{r}-1, l+2}\right\}=\mathcal{X}=\mathcal{X}_{\sigma} \rightarrow H_{\mathrm{b}}^{\tilde{r}-1, l+2},
$$

with $\mathcal{X}$ equipped with the squared norm

$$
\|u\|_{\mathcal{X}}^{2}=\|u\|_{H_{\mathrm{b}}^{\tilde{r}, l}}^{2}+\|P(\sigma) u\|_{H_{\mathrm{b}}^{\tilde{r}-1, l+2}}^{2}
$$

which makes it into a Hilbert space. While this might be a somewhat strange space, the space $\dot{\mathcal{C}}^{\infty}(X)$ of $\mathcal{C}^{\infty}$ functions vanishing to infinite order at $\partial X$ (i.e. Schwartz functions) is dense in it, as follows by approximating $u \in \mathcal{X}$ via $u_{\epsilon}=\Lambda_{\epsilon} u$, $\Lambda_{\epsilon} \in \Psi_{\mathrm{b}}^{-\infty,-\infty}(X), \epsilon \rightarrow 0$, where $\Lambda_{\epsilon}$ is uniformly bounded in $\Psi_{\mathrm{b}}^{0,0}(X)$, converging to Id in $\Psi_{\mathrm{b}}^{\delta^{\prime}, \delta^{\prime}}(X)$ for $\delta^{\prime}>0$; then $u_{\epsilon} \rightarrow u$ in $H_{\mathrm{b}}^{\tilde{r}, l}$ and moreover

$$
P(\sigma) u_{\epsilon}-P(\sigma) u=P(\sigma)\left(\Lambda_{\epsilon}-\mathrm{Id}\right) u=\left[P(\sigma), \Lambda_{\epsilon}\right] u+\left(\Lambda_{\epsilon}-\mathrm{Id}\right) P(\sigma) u \rightarrow 0
$$

in $H_{\mathrm{b}}^{\tilde{r}-1, l+2}$ as is immediate in case of the second term (for $P(\sigma) u \in H_{\mathrm{b}}^{\tilde{r}-1, l+2}$ ) and in the case of the first term as $\left[P(\sigma), \Lambda_{\epsilon}\right]=\left[P(0)+\sigma Q, \Lambda_{\epsilon}\right]$ is uniformly bounded in $\Psi_{\mathrm{b}}^{1,-2}$, converging to 0 in $\Psi_{\mathrm{b}}^{1+\delta^{\prime},-2+\delta^{\prime}}$ for $\delta^{\prime}>0$. This observation uses that $\sigma^{2}$ commutes with every operator, thus with $\Lambda_{\epsilon}$, which fact will also play a key role below.

Our main theorem concerns a uniform estimate about $P(\sigma)^{-1}$ in the above sense as $\sigma \rightarrow 0$. While $\mathcal{X}=\mathcal{X}_{\sigma}$ depends on $\sigma$, the interesting part of the bound on $P(\sigma)^{-1} f$ is its $H_{\mathrm{b}}^{\tilde{r}, l}$ norm (after all, $P(\sigma)$ applied to this is simply $f$ ); this is how we state the bound below.

Theorem 1.1 (See Theorem 4.5 for the general allowed $\tilde{r}$ ). Suppose that $|l+1|<$ $\frac{n-2}{2}$, and suppose that $P(0): H_{\mathrm{b}}^{\infty, l} \rightarrow H_{\mathrm{b}}^{\infty, l+2}$ has trivial nullspace, an assumption independent of $l$ in this range; see 1.4 .

There exist variable order b-Sobolev spaces $H_{\mathrm{b}}^{\tilde{r}, l}$ and $\sigma_{0}>0$ such that

$$
P(\sigma):\left\{u \in H_{\mathrm{b}}^{\tilde{r}, l}: P(\sigma) u \in H_{\mathrm{b}}^{\tilde{r}-1, l+2}\right\} \rightarrow H_{\mathrm{b}}^{\tilde{r}-1, l+2}
$$


is invertible for $0 \leq \sigma \leq \sigma_{0}$, with this inverse being the $\pm i 0$ resolvent of $P(\sigma)$ (with choice corresponding to that of $\tilde{r}$ ), and the norm of $P(\sigma)^{-1}$ as an element of $\mathcal{L}\left(H_{\mathrm{b}}^{\tilde{r}-1, l+2}, H_{\mathrm{b}}^{\tilde{r}, l}\right)$ is uniformly bounded in $\left[0, \sigma_{0}\right]$.

For instance, one can take any $\beta>0$,

$$
\tilde{r}=\hat{r}_{ \pm}(\beta)=\frac{1}{2}-(l+1) \pm \beta \frac{\tau_{\mathrm{b}}}{\left(\tau_{\mathrm{b}}^{2}+\left|\mu_{\mathrm{b}}\right|^{2}\right)^{1 / 2}},
$$

where $\tau_{\mathrm{b}}, \mu_{\mathrm{b}}$ are the b-dual variables defined above, $|$.$| the dual metric function of$ $g_{\partial X}$, and \pm corresponds to the $\pm i 0$ limits.

Indeed, the estimates hold in $|\sigma| \leq \sigma_{0}, \operatorname{Im}\left(\sigma^{2}\right) \geq 0$ (+ case), resp. $|\sigma| \leq \sigma_{0}$, $\operatorname{Im}\left(\sigma^{2}\right) \leq 0$ (- case)

Remark 1.2. For the general orders $\tilde{r}$, see Theorem 4.5

Remark 1.3. Notice that this theorem is lossless in terms of decay orders: for $\sigma=0$ the condition $P(\sigma) u \in H_{\mathrm{b}}^{\tilde{r}-1, l+2}$ together with $u \in H_{\mathrm{b}}^{\tilde{r}, l}$ implies $u \in H_{\mathrm{b}}^{\tilde{r}+1, l}$, and $P(0): H_{\mathrm{b}}^{\tilde{r}+1, l} \rightarrow H_{\mathrm{b}}^{\tilde{r}-1, l+2}$ is invertible. Thus, in this sense this is a very precise estimate. Also, the loss of one differentiability order relative to elliptic estimates is a standard real principal type/radial point phenomenon; while $P(0)$ is elliptic in $\Psi_{\mathrm{b}}^{2,-2}, P(\sigma)$ is not so for $\sigma \neq 0$ in $\Psi_{\mathrm{b}}^{2,0}$. For such $\sigma, P(\sigma)$ has real principal type/radial point estimates, mentioned above, due to a non-trivial characteristic set disjoint from the zero section of the ${ }^{\mathrm{sc}} T^{*} X$. This amounts to an infinite bfrequency phenomenon in view of (1.5), thus a b-differentiable order loss in terms of the $H_{\mathrm{b}}$ spaces. See Section 5 for the second microlocal scattering version.

Remark 1.4. In fact, $\sigma_{0}>0$ could be arbitrary if $P(\sigma)$ had no $L^{2}$-elements of its nullspace for any $\sigma$; this is a potential issue for $\sigma^{2}<0$ (thus not an issue for $\sigma$ real). The statement with arbitrary $\sigma_{0}$, under this additional assumption, follows easily from this theorem, and the limiting absorption principle for $\sigma^{2}>0$, resp. the elliptic theory for $\sigma^{2}<0$; or indeed it is a direct consequence of the proof of the Theorem.

Remark 1.5. We also have a version of the theorem with non-trivial nullspace. The result is then highly dimension dependent with the case $n \geq 5$ amounting to more or less standard Fredholm perturbation theory. We take this up in the final section of this paper, mostly concentrating on the most delicate $n=3$ case; here in the introduction we merely refer to Theorems 7.5 and 7.6 .

Remark 1.6. By standard Fredholm perturbation theory, if $P_{b}(\sigma)$ is a family of operators of the form discussed above for $P(\sigma)$ continuously depending on a parameter $b \in \mathbb{R}^{k}$ (i.e. $g$ depends continuously on $b$ in the class of asymptotically conic metrics, etc.), and $P_{0}(0)$ is invertible, then there is $\epsilon>0$ such that the same holds for $P_{b}(0)$ for $|b|<\epsilon$, i.e. the hypothesis of the theorem is perturbation stable.

Remark 1.7. The theorem is stated for operators acting on scalar functions. It also applies, with essentially the same proofs, if $P(\sigma)=P(0)+\sigma Q-\sigma^{2}$ is acting on sections of a vector bundle when $x^{-(n+2) / 2} P(0) x^{(n-2) / 2}$ (cf. 1.2 and (4.21)) is elliptic with positive scalar principal symbol and modulo $S^{-2-\delta}$ Diff $_{\mathrm{b}}^{2}$ (acting on sections of the bundle) is of the form

$$
\left(x D_{x}\right)^{2} \otimes \mathrm{Id}+\tilde{P}_{\partial X}+\left(\frac{n-2}{2}\right)^{2}
$$


with $\tilde{P}_{\partial X}$ being an elliptic differential operator on $\partial X$, which is non-negative, selfadjoint with respect to a Hermitian inner product, and $Q$ is of the class $S^{-2-\delta}$ Diff $_{\mathrm{b}}^{1}$, acting on sections of the bundle. Moreover, if $\frac{n-2}{2}$ is replaced by another constant, the same constant can be used in the statement of Theorem 1.1 in place of $\frac{n-2}{2}$.

Indeed, allowing $Q=Q_{0}+Q_{1}, Q_{0} \in x^{2} \operatorname{Diff}_{\mathrm{b}}^{1}$ and $Q_{1} \in S^{-2-\delta} \operatorname{Diff}_{\mathrm{b}}^{1}$ (so the coefficients of $Q$ do not decay relative to those of $x^{-(n+2) / 2} P(0) x^{(n-2) / 2}$ ) leaves the theorem valid, again with the proofs requiring only minor modification. While such a $Q$ affects the (skew-adjoint part of the extended) b-subprincipal symbol at the boundary, hence the argument of Section 3 see the term $\left(P(\sigma)^{*}-P(\sigma)\right) A^{*} A$ in (3.3), due to the prefactor $\sigma$, the subprincipal symbol can be made arbitrarily small (depending on the desired $l$ and $\tilde{r}$ ) by choosing $\sigma_{0}$ small, and then (a small constant times) the term in $a \mathrm{H}_{p} a$ corresponding to the first term of the right hand side of either of (3.8) and (3.18) dominates the new term in the principal symbol of $\left(P(\sigma)^{*}-P(\sigma)\right) A^{*} A$, so the rest of the argument is unaffected. Moreover, $Q_{0}$ now appears in the normal operator argument, but for the same reason as in the above symbolic argument, the principal symbol of the normal family has uniform estimates (in $\tau_{\mathrm{b}}$ ) of the same kind as if $Q_{0}$ vanished, which in particular gives the positivity/negativity of 4.22 for large $\tau_{\mathrm{b}}$ on the Mellin transform side, and then finally the positivity/negativity of 4.22 for finite $\tau_{\mathrm{b}}$ follows from the vanishing $Q_{0}$ case by the stability of the property of being positive/negative.

Indeed, an analogous argument shows that the conclusion of the theorem also holds if $x^{-(n+2) / 2} P(0) x^{(n-2) / 2}$ differs from $(1.7)$ by an operator $R=R_{0}+R_{1}$, $R_{0} \in x^{2} \operatorname{Diff}_{\mathrm{b}}^{1}, R_{1} \in S^{-2-\delta} \operatorname{Diff}_{\mathrm{b}}^{2}$, without formal self-adjointness assumptions on $R_{0}$ but instead assuming that $R_{0}$ is small in a suitable seminorm on $x^{2} \operatorname{Diff}_{\mathrm{b}}^{1}$. (Smallness needs to be assumed since there is no small parameter in front of $P(0)$, unlike $\sigma$ in the case of $Q$.)

Remark 1.8. It is straightforward to add an appropriate conormality statement (conormal to $\sigma=0$ ) to this using the commutation relation of $x D_{x}+\sigma D_{\sigma}$ with $P(\sigma)$. Notice that this is not quite a statement of $P(\sigma)^{-1}$ being conormal to 0 in weighted b-Sobolev spaces, rather a 'twisted version' of it.

Remark 1.9. This result can be extended to complex scaled operators, see 35 . Then it gives a similar uniform bound for the complex scaled resolvent down to 0 , again under the assumption of $P(0)$ having trivial nullspace as above.

Since one can take $\beta>0$ arbitrarily small, this in particular implies a (generalization of a) result of Bony and Häfner [5, see the end of the paper for the conversion to the scattering, thus on Euclidean space standard weighted Sobolev, function spaces:

Corollary 1.10. On long-range asymptotically Euclidean spaces, or more generally on Riemannian scattering spaces, of dimension $\geq 3$, the resolvent of the Laplacian satisfies that

$$
R\left(\sigma^{2} \pm i 0\right): H_{\mathrm{b}}^{1 / 2-(l+1)-1+\beta, l+2} \rightarrow H_{\mathrm{b}}^{1 / 2-(l+1)-\beta, l}
$$

is uniformly bounded (as a function of $\sigma$ ) on $\left[0, \sigma_{0}\right]$ for $|l+1|<\frac{n-2}{2}$ and $\beta>0$, and thus for all $s \in \mathbb{R}$ and for all $\beta>0$,

$$
R\left(\sigma^{2} \pm i 0\right): H_{\mathrm{sc}}^{s, 1+\beta} \rightarrow H_{\mathrm{sc}}^{s+2,-1-\beta}
$$


is uniformly bounded in the same sense, and more precisely, for the second microlocal spaces of Section 5, for $\beta>0, s \in \mathbb{R},|l+1|<\frac{n-2}{2}$,

$$
R\left(\sigma^{2} \pm i 0\right): H_{\mathrm{sc}}^{s, 1 / 2+\beta, l+2} \rightarrow H_{\mathrm{sc}}^{s+2,-1 / 2-\beta, l} .
$$

In fact, in Section 5 we show an even sharper version, with variable order weights, in Theorem 5.7 .

Remark 1.11. Other closely related works, which we only briefly mention here, include those of Bony and Häfner [4, 3] and of Vasy and Wunsch [42.

The basic idea of the proof of our main theorem is a positive (or negative) (twisted) commutator estimate in the b-pseudodifferential setting. This is a positivity result modulo compact operators, whose proof requires two ingredients. First, a positive principal symbol, capturing the b-differential order part of compactness; this has very much the flavor of the usual radial point estimate results on variable order Sobolev spaces, and is discussed in Section 3. Second, a positive normal operator, capturing the decay part of compactness. In general, this is a computation in a non-commutative algebra, namely an operator algebra on $\partial X$. However, one can arrange that the only operators involved are $\Delta_{\partial X}, x$ and $x D_{x}$, and $x$ only enters via overall multiplication by its powers, which means that in the commutator computation the effect will be a shift of $x D_{x}$ by an imaginary constant thanks to a conjugation. Thus, finally, on the Mellin transform side, using the spectral representation of $\Delta_{\partial X}$, one has to check the positivity of a scalar function, i.e. positivity in a commutative algebra. While this may be somewhat involved, it is in principle straightforward; we do this in Section 4 . This computation is simplified by a large parameter symbolic treatment (akin to semiclassical rescaling), which is how we proceed in Section 4. We remark that as we prove the general differential order $\tilde{r}$ version of the main theorem in Theorem 4.5 but we prove (and use) the normal operator positivity only for the special differential order, $(1.6)$, there is a slightly involved functional analytic argument in the first part of Section 4 that could be simplified if only the orders 1.6 were considered.

While this is a relatively standard approach, what makes it somewhat unusual in this case is that $P(\sigma)$ is regarded as effectively an element of $x^{2} \operatorname{Diff}_{\mathrm{b}}^{2}(X) \subset$ $\Psi_{\mathrm{b}}^{2,-2}(X)$, even though the spectral parameter does not lie in this space! However, in the (twisted) positive commutator estimate, it either disappears (since it is a multiple of the identity, thus commutes with every operator), or gives the correct sign (in the 'twisted term', involving $\left.P(\sigma)-P(\sigma)^{*}\right)$ when $\operatorname{Im}\left(\sigma^{2}\right)$ is non-zero but has the correct sign.

In Section 5 , we connect the results to scattering Sobolev spaces, which are the natural spaces for the limiting resolvents for positive spectral parameters. The 'interpolation' between the scattering and b- Sobolev spaces is given by second microlocalized scattering Sobolev spaces, with the second microlocalization taking place at the zero section; this is what brings the 'b-picture' into the scattering problem. We emphasize that this part is not necessary for proving Theorem 1.1 . rather it provides a single framework for understanding Theorem 1.1 and the more common positive energy limiting absorption results, and thus proves Corollary 1.10 along the way in a strengthened form in Theorem 5.7 .

Section 6 notes the changes that are necessary to adapt these arguments to Kerrlike spaces; the point being the lack of ellipticity in the interior of $X$. Finally, in 
Section 7 we discuss the estimates obtained when there are zero resonances both in the asymptotically Euclidean and in the Kerr settings.

The author is very grateful to Kiril Datchev, Dietrich Häfner, Rafe Mazzeo, Richard Melrose, Maciej Zworski and especially Peter Hintz and Jared Wunsch for fruitful discussions.

\section{BACKGROUND}

In this background section we discuss b-pseudodifferential operators. These are treated in great detail in Melrose's book 29] by describing their Schwartz kernels on a resolved double space. Here we follow [45, Section 5.6] by reducing their study to Hörmander's uniform class in local coordinates, with some modifications.

Thus, near a point on $\partial X$, consider local coordinates $(x, y), x \geq 0$ near $0, y$ in an open subset of $\mathbb{R}^{n-1}$; we in fact take all symbols to be compactly supported in the chart. In general, if one introduces logarithmic coordinates, $t=-\log x$, bpseudodifferential operators are just Hörmander's uniform pseudodifferential class locally in the corresponding cylindrical regions (positive real line in $t$ times a compact set in the $y$ variables). Thus, to define $\Psi_{\mathrm{bc}}^{m, 0}(X)$, locally one considers, with $\psi$ compactly supported, identically 1 near 0 , operators of the form

$$
B u(t, y)=(2 \pi)^{-n} \int e^{i\left[-\left(t-t^{\prime}\right) \tau_{\mathrm{b}}+\left(y-y^{\prime}\right) \cdot \mu_{\mathrm{b}}\right]} \psi\left(t-t^{\prime}\right) b\left(t, y, \tau_{\mathrm{b}}, \mu_{\mathrm{b}}\right) u\left(t^{\prime}, y^{\prime}\right) d t^{\prime} d y^{\prime},
$$

where $b \in S_{\infty}^{m}$, i.e.

$$
\left|\partial_{t}^{k} \partial_{y}^{\alpha} \partial_{\tau_{\mathrm{b}}}^{j} \partial_{\mu_{\mathrm{b}}}^{\beta} b\right| \leq C_{k \alpha j \beta}\left\langle\left(\tau_{\mathrm{b}}, \mu_{\mathrm{b}}\right)\right\rangle^{m-j-|\beta|} .
$$

(The minus sign in front of $\left(t-t^{\prime}\right)$ in the phase is added for consistency with the compactified notation below.) The localizer $\psi$ plays an important role; without it the Schwartz kernel would only decay polynomially in $\left|t-t^{\prime}\right|$, and to work on Sobolev spaces with polynomial gain in $x$ one needs Schwartz kernels decaying faster than $e^{-M\left|t-t^{\prime}\right|}$ for all $M$. Notice that $\partial_{t}$ can be replaced by $x \partial_{x}$ in this definition, thus this is exactly the symbol space on ${ }^{\mathrm{b}} T^{*} X$ as discussed in Section 5 ahead of Lemma 5.2

Notice that in terms of $x$, one could equivalently consider

$$
\tilde{B} u(x, y)=(2 \pi)^{-n} \int e^{i\left[\frac{x-x^{\prime}}{x} \tau_{\mathrm{b}}+\left(y-y^{\prime}\right) \cdot \mu_{\mathrm{b}}\right]} \tilde{\psi}\left(\frac{x-x^{\prime}}{x}\right) \tilde{b}\left(x, y, \tau_{\mathrm{b}}, \mu_{\mathrm{b}}\right) u\left(x^{\prime}, y^{\prime}\right) d t^{\prime} d y^{\prime},
$$

where $\tilde{\psi}$ is supported in $(-1 / 2,1 / 2)$, say, and identically 1 near 0 , and where the estimates on $\tilde{b}$ are those on $b$ pulled back via the map $\left(x, y, \tau_{\mathrm{b}}, \mu_{\mathrm{b}}\right) \mapsto\left(-\log x, y, \tau_{\mathrm{b}}, \mu_{\mathrm{b}}\right)$, so $\partial_{t}=-x \partial_{x}$, and this is simply the conormal estimate for $\tilde{b}$. Indeed, $\frac{x-x^{\prime}}{x}=$ $1-e^{t-t^{\prime}}$, so the support condition on $\tilde{\psi}$ is equivalent to a support condition on $\psi$ stated above, and similarly in the phase function $\frac{x-x^{\prime}}{x}=1-e^{t-t^{\prime}}$ is equivalent to $-\left(t-t^{\prime}\right)$ above, in the strong sense that at the critical set, $t=t^{\prime}$, the differentials are the same.

Then one adds to these residual in the symbolic, but not in the decay, sense terms $R$ satisfying estimates on their Schwartz kernel $K_{R}$

$$
\left|\partial_{t}^{k} \partial_{y}^{\alpha} \partial_{t^{\prime}}^{l} \partial_{y^{\prime}}^{\gamma} K_{R}\left(t, y, t^{\prime}, y^{\prime}\right)\right| \leq C_{k \alpha l \gamma N M}\left\langle y-y^{\prime}\right\rangle^{-N} e^{-M\left|t-t^{\prime}\right|}
$$

for all $l, k, \alpha, \gamma, M, N$; these are elements of the Hörmander class $\Psi_{\infty}^{-\infty}$, but we impose stronger, exponential, decay in $\left|t-t^{\prime}\right|$. The full local version of $\Psi_{\mathrm{bc}}^{m, 0}$ then 
consists of operators $A$ of the form $A=B+R$. One can then transplant these to a manifold with boundary via local coordinates, much as in the standard setting; we refer to [45, Section 5.6] for a detailed discussion.

We also define $\Psi_{\mathrm{bc}}^{m, l}(X)=x^{-l} \Psi_{\mathrm{bc}}^{m, 0}(X)$. Note that this is the opposite, in terms of the sign of $l$, of Melrose's order convention, but it is helpful as the space of operators gets larger with increasing $m$ as well as with increasing $l$.

Recall that in the standard sense, namely considering Hörmander's uniform class with symbolic behavior in the dual variables, a symbol $b$ of order $m$ is called classical if it has an asymptotic expansion in homogeneous (with respect to dilations in $\left.\left(\tau_{\mathrm{b}}, \mu_{\mathrm{b}}\right)\right)$ symbols of order $m-j(j \in \mathbb{N})$, in the sense that the difference between the terms of the expansion with $j \leq k-1$ and $b$ is in $S_{\infty}^{m-k}$. This can also be defined instead as a classical conormality statement when the fibers of ${ }^{\mathrm{b}} T^{*} X$ (which are vector spaces) are radially compactified (as discussed in the introduction for Euclidean space; the fibers are such). If $m=0$ classicality is thus a uniform version (in the base variables $(t, y)$ ) of smoothness on ${ }^{\mathrm{b}} T^{*} X^{\circ} X$. The general case reduces to this after factoring out the $-m$ th power of a defining function of the new boundary, fiber infinity; one can take this defining function (away from the zero section) to be $\left(\tau_{\mathrm{b}}^{2}+\left|\mu_{\mathrm{b}}\right|^{2}\right)^{-1 / 2}$, with $\left|\mu_{\mathrm{b}}\right|^{2}$ the squared length with respect any dual metric on $\partial X$, much as $r^{-1}$ could be taken as the defining function of the boundary of the radial compactification (away from 0). (If one wants to allow the zero section, resp. the origin, one can take $\left(\tau_{\mathrm{b}}^{2}+\left|\mu_{\mathrm{b}}\right|^{2}+1\right)^{-1 / 2}$, resp. $\left(r^{2}+1\right)^{-1 / 2}$. From the perspective of $X$, i.e. using $x$ in place of $t$, this amounts to conormality at $\partial X$ (more precisely at $\overline{\mathrm{b}} T^{*} \partial X X$ ), with values in classical symbols; thus, $x \partial_{x}$ and $\partial_{y_{j}}$ preserve the smoothness in the fibers.

However, since $X$ itself has a boundary it also makes sense of classicality at $\partial X$. The fully classical operators (i.e. classical both in the symbolic and in the boundary asymptotic expansion sense) are then ones possessing an expansion in Taylor series in $x$, i.e. in terms of exponentials $e^{-t}$. For operators of order $(0,0)$ thus full classicality amounts to both $b$ being $\mathcal{C}^{\infty}$ in the local coordinate version of $\overline{\mathrm{b}} T^{*} X$, and the residual term (which is trivial in the symbolic sense) possessing an expansion in Taylor series in $x$.

Just like there was a partial classicality in the symbolic sense, with conormal behavior in the base, there is also a partial classicality in the base sense, with symbolic behavior in the differential sense; for elements of $\Psi_{\mathrm{bc}}^{m, 0}$ this amounts to $b$ being smooth (with values in symbols) in $(x, y)$, i.e. having a Taylor series expansion in $x$, plus again the residual term being smooth in $x$. A limited version of this partial conormality plays a role in defining the normal operator below.

The space $\Psi_{\mathrm{bc}}^{\infty, \infty}(X)=\cup_{m, l \in \mathbb{R}} \Psi_{\mathrm{bc}}^{m, l}(X)$ is a filtered $*$-algebra, with $A \in \Psi_{\mathrm{bc}}^{m, l}(X)$, $A^{\prime} \in \Psi_{\mathrm{bc}}^{m^{\prime}, l^{\prime}}(X)$ implying $A A^{\prime} \in \Psi_{\mathrm{b}}^{m+m^{\prime}, l+l^{\prime}}(X)$ and $A^{*} \in \Psi_{\mathrm{bc}}^{m, l}(X)$ where the adjoint is taken with respect to any non-degenerate (positive) b-density, or indeed any non-degenerate polynomial multiple of this. In local coordinates, a b-density has the form $a \frac{\left|d x d y_{1} \ldots d y_{n-1}\right|}{x}$, with $a>0$ (on $X$, including at $\partial X$ ) meaning that this b-density is positive; notice that this is $a\left|d t d y_{1}, \ldots d y_{n-1}\right|$; the polynomial multiples take the form $a x^{p} \frac{\left|d x d y_{1} \ldots d y_{n-1}\right|}{x}$ for some $p \in \mathbb{R}$, which include scattering densities, such as densities of asymptotically conic metrics, for which $p=-n$. (So the scattering $L^{2}$-space is $L_{\mathrm{sc}}^{2}(X)=x^{n / 2} L_{\mathrm{b}}^{2}(X)$.) 
Furthermore, there is a principal symbol map

$$
\sigma_{m, l}: \Psi_{\mathrm{bc}}^{m, l} \rightarrow S^{m, l} / S^{m-1, l}
$$

for classical operators one often identifies $\sigma_{m, l}(A)$ with a homogeneous degree $m$ function on ${ }^{\mathrm{b}} T^{*} X \backslash o$ without further comments. The principal symbol captures $\Psi_{\mathrm{bc}}^{m, l}(X)$ modulo $\Psi_{\mathrm{bc}}^{m-1, l}(X)$, so if the principal symbol of $A \in \Psi_{\mathrm{bc}}^{m, l}(X)$ vanishes, then $A \in \Psi_{\mathrm{bc}}^{m-1, l}(X)$. As usual, this principal symbol is a $*$-algebra homomorphism, so

$$
\sigma_{m+m^{\prime}, l+l^{\prime}}\left(A A^{\prime}\right)=\sigma_{m, l}(A) \sigma_{m^{\prime}, l^{\prime}}\left(A^{\prime}\right) .
$$

This algebra is thus commutative to leading order in the differential sense, i.e. $\left[A, A^{\prime}\right] \in \Psi_{\mathrm{bc}}^{m+m^{\prime}-1, l+l^{\prime}}(X)$, but there is no gain in the decay order. Furthermore, one can compute the principal symbol of $\left[A, A^{\prime}\right]$ as an element of $\Psi_{\mathrm{bc}}^{m+m^{\prime}-1, l+l^{\prime}}(X)$ (rather than just as an element of $\left[A, A^{\prime}\right] \in \Psi_{\mathrm{bc}}^{m+m^{\prime}, l+l^{\prime}}(X)$, in which sense it vanishes by the algebra homomorphism property); it is given by the usual Hamilton vector field expression:

$$
\sigma_{m+m^{\prime}-1, l+l^{\prime}}\left(\left[A, A^{\prime}\right]\right)=\frac{1}{i} \mathrm{H}_{a} a^{\prime}, a=\sigma_{m}(A), a^{\prime}=\sigma_{m^{\prime}}\left(A^{\prime}\right) .
$$

For $l=0, \mathrm{H}_{a}$ is a b-vector field on ${ }^{\mathrm{b}} T^{*} X$, i.e. is tangent to ${ }^{\mathrm{b}} T_{\partial X}^{*} X$ (and in general it simply has an extra weight factor); indeed in local coordinates it takes the form

$$
\begin{aligned}
& \left(\partial_{\tau_{\mathrm{b}}} a\right)\left(x \partial_{x}\right)-\left(x \partial_{x} a\right) \partial_{\tau_{\mathrm{b}}}+\sum_{j}\left(\left(\partial_{\left(\mu_{\mathrm{b}}\right)_{j}} a\right) \partial_{y_{j}}-\left(\partial_{y_{j}} a\right) \partial_{\left(\mu_{\mathrm{b}}\right)_{j}}\right) \\
& =\left(-\partial_{\tau_{\mathrm{b}}} a\right) \partial_{t}-\partial_{t} a\left(-\partial_{\tau_{\mathrm{b}}}\right)+\sum_{j}\left(\left(\partial_{\left(\mu_{\mathrm{b}}\right)_{j}} a\right) \partial_{y_{j}}-\left(\partial_{y_{j}} a\right) \partial_{\left(\mu_{\mathrm{b}}\right)_{j}}\right),
\end{aligned}
$$

where the - signs in the $\partial_{t}$-version correspond to $\tau_{\mathrm{b}} \frac{d x}{x}=-\tau_{\mathrm{b}} d t$; notice that the second line is the standard form of the Hamilton vector field taking into account that $\tau_{\mathrm{b}}$ is the negative of the canonical dual coordinate of $t$.

In order to capture decay, one can use the normal operator of $A \in \Psi_{\mathrm{bc}}^{m, 0}(X)$, which is defined if $A$ is classical in the base variable $x$ modulo $\Psi_{\mathrm{bc}}^{m,-\delta}(X)$, i.e. $A$ differs from a dilation invariant operator $N(A)$ on $(0, \infty) \times \partial X$, with a neighborhood $\left[0, x_{0}\right) \times \partial X$ of $\partial X$ being identified with this model locally, by an element of $\Psi_{\mathrm{bc}}^{m,-\delta}(X)$. The main advantage of this is that $N(A)$ can be analyzed via the Mellin transform in $x$, i.e. the Fourier transform in $-t$, thanks to its dilation invariance; these reduce the analysis to that of a family of pseudodifferential operators $\hat{N}(A)$ on $\partial X$. Note that the latter form a non-commutative algebra, thus this a more complicated object than the principal symbol. For general $l$, one can instead consider the rescaled normal operator $N\left(x^{l} A\right)$ for similar effect. For example, if $g$ is a sc-metric which is asymptotically conic, the normal operator of $\Delta_{g}$ is simply that of the Laplacian of the conic model metric.

When working with variable (differential, in this case) order operators, i.e. $m$ is a smooth function of $\left(x, y, \tau_{\mathrm{b}}, \mu_{\mathrm{b}}\right)$ which is homogeneous of degree zero in $\left(\tau_{\mathrm{b}}, \mu_{\mathrm{b}}\right)$, it is also necessary to generalize the definition by allowing losses $\delta \in[0,1 / 2)$ :

$$
\left|\partial_{t}^{k} \partial_{y}^{\alpha} \partial_{\tau_{\mathrm{b}}}^{j} \partial_{\mu_{\mathrm{b}}}^{\beta} b\right| \leq C_{k \alpha j \beta}\left\langle\left(\tau_{\mathrm{b}}, \mu_{\mathrm{b}}\right)\right\rangle^{m-j-|\beta|+\delta(k+|\alpha|+j+|\beta|)} ;
$$


this class of symbols would have a subscript $\delta$, as would do the corresponding class of pseudodifferential operators $\Psi_{\mathrm{bc}, \delta}^{m, 0}$; see [45] where this is discussed throughout the paper in various settings. The need for these (or slightly different versions) arises from the logarithmic terms coming from differentiating expressions like $\left\langle\left(\tau_{\mathrm{b}}, \mu_{\mathrm{b}}\right)\right\rangle^{m\left(x, y, \tau_{\mathrm{b}}, \mu_{\mathrm{b}}\right)}$; the latter is an example of an elliptic order $m$ symbol. These symbols and the corresponding operators work completely analogously to the $\delta=0$ case considered above, except that the principal symbol is defined in $S_{\delta}^{m, l} / S_{\delta}^{m-1+2 \delta, l}$, and the commutator of two operators as above is in $\Psi_{\mathrm{bc}, \delta}^{m+m^{\prime}-1+2 \delta, l+l^{\prime}}(X)$, and thus its principal symbol is computed modulo $S_{\delta}^{m+m^{\prime}-1+4 \delta, l+l^{\prime}}$. It is important to keep in mind that for our purposes $\delta>0$ can always be taken arbitrarily small.

\section{Symbolic estimate}

We start the proof of the main theorem by proving a uniform (in $\sigma$ ) symbolic estimate, which does not yet come with compact error terms. We first show it with a special choice of $\tilde{r}$, which makes the computation completely explicit, see Proposition 3.1, and later on we generalize to a more geometric condition on the differential order $\tilde{r}$, see Proposition 3.5

Proposition 3.1. Let $l \in \mathbb{R}, \beta>0$ and let

$$
\tilde{r}=\frac{1}{2}-(l+1) \pm \beta \frac{\tau_{\mathrm{b}}}{\left(\tau_{\mathrm{b}}^{2}+\left|\mu_{\mathrm{b}}\right|^{2}\right)^{1 / 2}},
$$

where |.| is the dual metric function of $g_{\partial X}$.

For $\sigma$ in a compact subset of $[0, \infty)$ and $0<K<\beta$ there exists $C>0$ such that for all $u \in H_{\mathrm{b}}^{\tilde{r}-K, l}$ with $P(\sigma) u \in H_{\mathrm{b}}^{\tilde{r}-1, l+2}$, we have $u \in H_{\mathrm{b}}^{\tilde{r}, l}$ and

$$
\|u\|_{H_{\mathrm{b}}^{\tilde{r}, l}} \leq C\left(\|P(\sigma) u\|_{H_{\mathrm{b}}^{\tilde{r}-1, l+2}}+\|u\|_{H_{\mathrm{b}}^{\tilde{r}-K, l}}\right) .
$$

Remark 3.2. Unlike in the main theorem, Theorem 1.1, there are no conditions on the decay order $l$. The latter only enter if one wants to improve (weaken) the decay order $l$ of the error term, $\|u\|_{H_{\mathrm{b}}^{\tilde{r}-K, l}}$, as we do in the next section.

Remark 3.3. By the density of $\dot{\mathcal{C}}^{\infty}(X)$ in $\mathcal{X}=\mathcal{X}_{\sigma}$, it in fact suffices to prove the uniform estimate (3.1) for $u \in \dot{\mathcal{C}}^{\infty}(X)$ for the purposes of proving the main theorem. Here we prove the significantly stronger statement made above; this has the flavor of propagation of singularities (really, regularity). We note that if we only want to prove the estimate for $u \in \dot{\mathcal{C}}^{\infty}(X)$, the regularization argument below is in fact unnecessary, shortening the proof.

Remark 3.4. Note that for any $\tilde{r}^{\prime},\|u\|_{H_{\mathrm{b}}^{\tilde{r}-K, l}}$ can be bounded by an arbitrarily small multiple of $\|u\|_{H_{\mathrm{b}}^{\tilde{r}, l}}$ plus a large multiple of $\|u\|_{H_{\mathrm{b}}^{\tilde{r}^{\prime}, l}}$, and the former can be absorbed into the left hand side of (3.1), so in fact 3.1 holds with $\|u\|_{H_{\mathrm{b}}^{\tilde{r}-K, l}}$ replaced by $\|u\|_{H_{\mathrm{b}}^{\tilde{r}^{\prime}, l}}$. Nonetheless, this does not change the need for the a priori assumption $u \in H_{\mathrm{b}}^{\tilde{r}-K, l}$ for some $0<K<\beta$; in the form (3.1) is stated, one can make it implicit by saying that the inequality holds provided the right hand side is finite. Thus, in the setting of Remark 3.3 , thus working with $\mathcal{X}$, it actually suffices to prove the estimate (3.1) for any single value of $K>0$, and then the stated version follows immediately. 
The rest of this section, until the statement of Proposition 3.5. consists of the proof of Proposition 3.1 .

The estimate (3.1) arises from a positive commutator argument. Although $P(\sigma) \in \operatorname{Diff}_{\mathrm{b}}^{2}(X)$ only, it is of the form

$$
P(\sigma)=P(0)+\sigma Q-\sigma^{2}, \quad P(0) \in x^{2} \operatorname{Diff}_{\mathrm{b}}^{2}(X), Q \in S^{-2-\delta} \operatorname{Diff}_{\mathrm{b}}^{1}(X)
$$

and $\sigma^{2}$, by virtue of being a multiple of the identity operator, commutes with every operator. Thus, the argument works as if $P(\sigma)$ were in $x^{2} \operatorname{Diff}_{\mathrm{b}}^{2}(X) \subset \Psi_{\mathrm{b}}^{2,-2}(X)$ (in a uniform manner in $\sigma$ with $\sigma$ in a fixed compact subset of $\mathbb{C}$ ), though with a bit of care. Moreover, not only is $\sigma Q$ subprincipal (by being a first order operator), but it has an extra $x^{\delta}$ vanishing at $\partial X$, so its principal symbol vanishes at $\partial X$.

While dealing with $P(0)$ it is usually convenient to conjugate it by $x^{(n-1) / 2}$ and multiply by $x^{-2}$ from the left; this gives a formally self-adjoint operator relative to the b-density $x^{n} d g$. However, in view of $\sigma^{2}$, we avoid this conjugation, for $\sigma^{2}$ is both symmetric relative to $d g$ and is a multiple of the identity operator. We thus use $d g$ for the density defining the inner product, and use it also for the $L^{2}$-space even in the b-algebra below; notice that thus

$$
L^{2}=x^{n / 2} L_{\mathrm{b}}^{2}
$$

with $L_{\mathrm{b}}^{2}$ defined with respect to any non-degenerate smooth b-density (i.e. locally a positive smooth multiple of $\frac{d x}{x} d y$ ) since such a density is, up to an overall positive smooth factor, $x^{n}$ times $d g$. Thus, $P(\sigma)$ is effectively (though not actually: actually it is merely in $\Psi_{\mathrm{b}}^{2,0}(X)$ due to $\left.\sigma^{2}\right)$ in $\Psi_{\mathrm{b}}^{2,-2}(X)$; taking $A \in \Psi_{\mathrm{b}}^{\tilde{r}-1 / 2, l+1}(X)$, with $A^{*}=A$, we have $i\left[P(\sigma), A^{*} A\right] \in \Psi_{\mathrm{b}}^{2 \tilde{r}, 2 l}(X)$; we also have for $\sigma \in \mathbb{R}, P(\sigma)=P(\sigma)^{*}$. In fact, if we allow $\operatorname{Im} \sigma \neq 0$, then depending on the $\operatorname{sign}$ of $\operatorname{Im} \sigma$, we obtain $i$ times a positive or negative term; this sign matches the sign below for the matching choice of weight, and thus function space; see the end of the section. Thus,

$$
\begin{aligned}
& i\left(P(\sigma)^{*} A^{*} A-A^{*} A P(\sigma)\right)=i\left(P(\sigma)^{*}-P(\sigma)\right) A^{*} A+i\left[P(\sigma), A^{*} A\right], \\
& {\left[P(\sigma), A^{*} A\right] \in \Psi_{\mathrm{b}}^{2 \tilde{r}, 2 l}(X),}
\end{aligned}
$$

with principal symbol $2 a \mathrm{H}_{p} a$, where $p$ is the principal symbol of $P(0)$. Below we arrange that this has a definite sign.

This is done by a global version of real principal type and radial point estimates. These were discussed, in the scattering setting, by Melrose in [27; the present version essentially presents a globalized version of [45, which in turn is based on [40] and [2].

Recall that the b-dual variable of $x$ is written as $\tau_{\mathrm{b}}$, that of $y_{j}$ as $\left(\mu_{\mathrm{b}}\right)_{j}$, so covectors are written as

$$
\tau_{\mathrm{b}} \frac{d x}{x}+\sum_{j}\left(\mu_{\mathrm{b}}\right)_{j} d y_{j}
$$

and a defining function of fiber infinity of $\overline{{ }^{\mathrm{b}} T^{*}} X$ is $\tilde{\rho}=\left(\tau_{\mathrm{b}}^{2}+\left|\mu_{\mathrm{b}}\right|^{2}\right)^{-1 / 2}$, with $\left|\mu_{\mathrm{b}}\right|^{2}$ the squared length with respect to any (dual) metric on $\partial X$, but here we take this to be $g_{\partial X}$, see Section 2 and also Section 5 . Then we take

$$
a=x^{-l-1}\left(\tau_{\mathrm{b}}^{2}+\left|\mu_{\mathrm{b}}\right|^{2}\right)^{(\tilde{r}-1 / 2) / 2} \psi(x),
$$

where $\tilde{r}$ is a function of the homogeneous degree zero expression $\tau_{\mathrm{b}} /\left(\tau_{\mathrm{b}}^{2}+\left|\mu_{\mathrm{b}}\right|^{2}\right)^{1 / 2}$ on ${ }^{\mathrm{b}} T^{*} X$ (minus the zero section), which is monotone along the Hamilton flow of $p$ at $\partial X$, and where $\psi \geq 0$ is identically 1 near 0 , and is supported sufficiently 
close to 0 (so that the collar neighborhood decomposition is still valid, and later on so that the dynamical behavior is unchanged). Concretely, depending on the incoming/outgoing choice ( $\pm i 0$ limits), we take, with $\beta>0$,

$$
\tilde{r}=\frac{1}{2}-(l+1) \pm \beta \frac{\tau_{\mathrm{b}}}{\left(\tau_{\mathrm{b}}^{2}+\left|\mu_{\mathrm{b}}\right|^{2}\right)^{1 / 2}} .
$$

Notice that on $\operatorname{supp} d \psi, x$ is bounded away from 0 , thus we have a priori elliptic estimates. Also, $a$ is to be understood as an amplitude, cut off from the zero section, but for a symbolic argument, such as those below, any additional term generated by this cutoff is of order $-\infty$, thus can be absorbed into the error.

With $p_{\partial}=x^{2}\left(\tau_{\mathrm{b}}^{2}+\left|\mu_{\mathrm{b}}\right|^{2}\right)$ the restriction of $p$ to $\partial X$, extended using the local product structure as an $x$-independent function, $\mathrm{H}_{p}-\mathrm{H}_{p_{\partial}}$ is $x^{2+\delta}$ times a homogeneous (with respect to dilations in the fibers of ${ }^{\mathrm{b}} T^{*} X$ ) degree 1 vector field tangent (in the sense of being a b-vector field with symbolic order 0 coefficients) to ${ }^{\mathrm{b}} T_{\partial X}^{*} X$, i.e. is $x^{2+\delta}$ times a linear combination of $x \partial_{x}, \partial_{y_{j}}$ with homogeneous degree 1 , and $\partial_{\tau_{\mathrm{b}}}, \partial_{\left(\mu_{\mathrm{b}}\right)_{j}}$ with homogeneous degree 2 coefficients, with these coefficients symbolic of order 0, i.e. remain bounded under applications of $x \partial_{x}, \partial_{y_{j}}, \partial_{\tau_{\mathrm{b}}}, \partial_{\left(\mu_{\mathrm{b}}\right)_{j}}$. (Here homogeneity is used to encode the regularity at fiber infinity.) Furthermore,

$$
\begin{aligned}
\mathrm{H}_{p_{\partial}} & =\left(\partial_{\tau_{\mathrm{b}}} p\right) x \partial_{x}-\left(x \partial_{x} p\right) \partial_{\tau_{\mathrm{b}}}+x^{2} \mathrm{H}_{\left|\mu_{\mathrm{b}}\right|^{2}} \\
& =2 x^{2} \tau_{\mathrm{b}} x \partial_{x}-2 x^{2}\left(\tau_{\mathrm{b}}^{2}+\left|\mu_{\mathrm{b}}\right|^{2}\right) \partial_{\tau_{\mathrm{b}}}+x^{2} \mathrm{H}_{\left|\mu_{\mathrm{b}}\right|^{2}},
\end{aligned}
$$

so

$$
\begin{aligned}
\mathrm{H}_{p}=2 x^{2}\left(\tau_{\mathrm{b}}+x^{\delta} q_{0}\right) x \partial_{x} & -2 x^{2}\left(\tau_{\mathrm{b}}^{2}+\left|\mu_{\mathrm{b}}\right|^{2}+x^{\delta} \tilde{q}_{0}\right) \partial_{\tau_{\mathrm{b}}} \\
& +x^{2} \mathbf{H}_{\left|\mu_{\mathrm{b}}\right|^{2}}+\sum_{j=1}^{n-1} x^{2+\delta}\left(q_{j} \partial_{y_{j}}+\tilde{q}_{j} \partial_{\left(\mu_{\mathrm{b}}\right)_{j}}\right),
\end{aligned}
$$

with $q_{j}$ homogeneous of degree $1, \tilde{q}_{j}$ homogeneous of degree 2 , symbolic of order 0 in $x$. As $\mathrm{H}_{\left|\mu_{\mathrm{b}}\right|^{2}}$ annihilates $a$ for the above choices,

$$
\begin{aligned}
\mathrm{H}_{p_{\partial}} a=2 x^{2} x^{-l-1}( & -(l+1) \tau_{\mathrm{b}}\left(\tau_{\mathrm{b}}^{2}+\left|\mu_{\mathrm{b}}\right|^{2}\right)^{(\tilde{r}-1 / 2) / 2} \psi(x) \\
& -(\tilde{r}-1 / 2) \tau_{\mathrm{b}}\left(\tau_{\mathrm{b}}^{2}+\left|\mu_{\mathrm{b}}\right|^{2}\right)^{(\tilde{r}-1 / 2) / 2} \psi(x) \\
& -\left(\partial_{\tau_{\mathrm{b}}} \tilde{r}\right)\left(\tau_{\mathrm{b}}^{2}+\left|\mu_{\mathrm{b}}\right|^{2}\right)^{1+(\tilde{r}-1 / 2) / 2} \log \left(\tau_{\mathrm{b}}^{2}+\left|\mu_{\mathrm{b}}\right|^{2}\right) \psi(x) \\
& \left.+x \psi^{\prime}(x) \tau_{\mathrm{b}}\left(\tau_{\mathrm{b}}^{2}+\left|\mu_{\mathrm{b}}\right|^{2}\right)^{(\tilde{r}-1 / 2) / 2}\right), \\
& \\
\partial_{\tau_{\mathrm{b}}} \tilde{r}=( & \pm \beta)\left(\tau_{\mathrm{b}}^{2}+\left|\mu_{\mathrm{b}}\right|^{2}\right)^{-3 / 2}\left(\tau_{\mathrm{b}}^{2}+\left|\mu_{\mathrm{b}}\right|^{2}-\tau_{\mathrm{b}}^{2}\right) \\
= & ( \pm \beta)\left|\mu_{\mathrm{b}}\right|^{2}\left(\tau_{\mathrm{b}}^{2}+\left|\mu_{\mathrm{b}}\right|^{2}\right)^{-3 / 2} .
\end{aligned}
$$

On the other hand,

$$
\begin{aligned}
\mathrm{H}_{p} a-\mathrm{H}_{p_{\partial}} a=2 x^{2} x^{-l-1} x^{\delta}\left(\left(\tau_{\mathrm{b}}^{2}\right.\right. & \left.+\left|\mu_{\mathrm{b}}\right|^{2}\right)^{(\tilde{r}-1 / 2) / 2}\left(f_{0} \psi+\tilde{f}_{0} x \psi^{\prime}\right) \\
& +\psi f_{1}\left(\tau_{\mathrm{b}}^{2}+\left|\mu_{\mathrm{b}}\right|^{2}\right)^{(\tilde{r}-1 / 2) / 2-1} \\
& \left.+\psi f_{2}\left(\tau_{\mathrm{b}}^{2}+\left|\mu_{\mathrm{b}}\right|^{2}\right)^{(\tilde{r}-1 / 2) / 2} \log \left(\tau_{\mathrm{b}}^{2}+\left|\mu_{\mathrm{b}}\right|^{2}\right)\right),
\end{aligned}
$$


with $f_{0}, \tilde{f}_{0}$ homogeneous of degree $1, f_{1}$ homogeneous of degree $3, f_{2}$ homogeneous of degree 1. Thus

$$
\begin{gathered}
\mathrm{H}_{p} a=-2( \pm \beta) x^{-l+1} \psi(x)\left(\tau_{\mathrm{b}}^{2}+\left|\mu_{\mathrm{b}}\right|^{2}\right)^{(\tilde{r}-3 / 2) / 2} \\
\cdot\left(\tau_{\mathrm{b}}^{2}+x^{\delta} f_{1}^{\sharp}+\left(\left|\mu_{\mathrm{b}}\right|^{2}+x^{\delta} f_{2}^{\sharp}\right) \log \left(\tau_{\mathrm{b}}^{2}+\left|\mu_{\mathrm{b}}\right|^{2}\right)\right)+e, \\
e=2 x^{2} x^{-l-1} x \psi^{\prime}(x)\left(\tau_{\mathrm{b}}+x^{\delta} f_{0}^{\sharp}\right)\left(\tau_{\mathrm{b}}^{2}+\left|\mu_{\mathrm{b}}\right|^{2}\right)^{(\tilde{r}-1 / 2) / 2}
\end{gathered}
$$

with $f_{0}^{\sharp}$ homogeneous of degree $1, f_{1}^{\sharp}, f_{2}^{\sharp}$ homogeneous of degree 2 . The first term in $\mathrm{H}_{p} a$ is the 'main term', while the second term, $e$, is the 'error term' and it is controlled by a priori elliptic estimates in $X^{\circ}$ near $\partial X$, so its sign is irrelevant for considerations below. Returning to the main term, for $\tau_{\mathrm{b}}^{2}+\left|\mu_{\mathrm{b}}\right|^{2}>2$ and $x$ sufficiently small, which can be arranged by making $\operatorname{supp} \psi$ sufficiently small, it is negative, resp. positive, depending on whether the sign in front of $\beta$ is positive, resp. negative, since

$$
\tau_{\mathrm{b}}^{2}+x^{\delta} f_{1}^{\sharp}+\left(\left|\mu_{\mathrm{b}}\right|^{2}+x^{\delta} f_{2}^{\sharp}\right) \log \left(\tau_{\mathrm{b}}^{2}+\left|\mu_{\mathrm{b}}\right|^{2}\right) \geq \tau_{\mathrm{b}}^{2}+\left|\mu_{\mathrm{b}}\right|^{2}-C x^{\delta}\left(\tau_{\mathrm{b}}^{2}+\left|\mu_{\mathrm{b}}\right|^{2}\right)
$$

there. Notice that this is slightly stronger, away from the radial sets (where $\mu_{\mathrm{b}}=$ 0 ), than the standard positive commutator estimate due to the presence of the logarithmic factor, but we do not need to explicitly take advantage of this, though we do need to point out that the expression in the big parentheses is a symbol of order $2+\delta^{\prime}$ for all $\delta^{\prime}>0$, and thus the usual arguments go through, as discussed in the non-radial point setting in e.g. in the appendix of [2], see also [39] for earlier work in which the logarithmic improvement played an important role. We explicitly point out the expression in the big parentheses in 3.8 is not only a symbol of order $2+\delta^{\prime}$ for all $\delta^{\prime}>0$, but corresponding to $(3.9)$ it has 'elliptic' positive lower bounds in an order 2 sense, thus the standard argument shows that its positive square root is a symbol of order $1+\delta^{\prime}$ for all $\delta^{\prime}$ with an order 1 'elliptic' positive lower bound, hence the corresponding term in $2 a \mathrm{H}_{p} a$ is

$$
b_{0}^{2}=4 \beta x^{-2 l} \psi(x)^{2}\left(\tau_{\mathrm{b}}^{2}+\left|\mu_{\mathrm{b}}\right|^{2}\right)^{\tilde{r}-1}\left(\tau_{\mathrm{b}}^{2}+x^{\delta} f_{1}^{\sharp}+\left(\left|\mu_{\mathrm{b}}\right|^{2}+x^{\delta} f_{2}^{\sharp}\right) \log \left(\tau_{\mathrm{b}}^{2}+\left|\mu_{\mathrm{b}}\right|^{2}\right)\right)
$$

with $b_{0}$ the non-negative square root which is in $x^{-l} S^{\tilde{r}+\delta^{\prime}}$ for all $\delta^{\prime}>0$ with a positive elliptic lower bound in $x^{-l} S^{\tilde{r}}$.

In fact, as usual, cf. [27, 40, in order to prove Proposition 3.1 (as opposed to the weaker version stated in Remark 3.3 one needs a family of operators $A_{\epsilon}$, where $\epsilon \in[0,1]$ is a regularization parameter, with, for $0<K<\beta$ as in the statement of the proposition, $A_{\epsilon} \in \Psi_{\mathrm{b}}^{\tilde{r}-1 / 2-K, l+1}(X)$ for $\epsilon>0,\left\{A_{\epsilon}: \epsilon \in[0,1]\right\}$ uniformly bounded in $\Psi_{\mathrm{b}}^{\tilde{r}-1 / 2, l+1}(X)$, converging to $A_{0}$ as $\epsilon \rightarrow 0$ in slightly weaker topologies, that of $\Psi_{\mathrm{b}}^{\tilde{r}-1 / 2+\delta^{\prime}, l+1}(X)$, for all $\delta^{\prime}>0$. (This convergence implies strong convergence in bounded operators between b-Sobolev spaces of appropriately shifted orders.) Concretely, we can take

$$
a_{\epsilon}=a \phi_{\epsilon}\left(\tilde{\rho}^{-1}\right), \quad \phi_{\epsilon}(s)=(1+\epsilon s)^{-K}, \quad \tilde{\rho}=\left(\tau_{\mathrm{b}}^{2}+\left|\mu_{\mathrm{b}}\right|^{2}\right)^{-1 / 2} .
$$

Note that

$$
\phi_{\epsilon}^{\prime}(s)=-K \epsilon(1+\epsilon s)^{-1} \phi_{\epsilon},
$$


so, with $f_{3}^{\sharp}$ homogeneous of degree 2 with symbolic order 0 coefficients (in the same sense as above),

$$
\begin{aligned}
\mathrm{H}_{p} \phi_{\epsilon}\left(\tilde{\rho}^{-1}\right) & =-K \epsilon\left(1+\epsilon \tilde{\rho}^{-1}\right)^{-1} \phi_{\epsilon}\left(\tilde{\rho}^{-1}\right) \mathrm{H}_{p} \tilde{\rho}^{-1} \\
& =x^{2} K \epsilon\left(1+\epsilon\left(\tau_{\mathrm{b}}^{2}+\left|\mu_{\mathrm{b}}\right|^{2}\right)^{1 / 2}\right)^{-1}(-2)\left(\left(\tau_{\mathrm{b}}^{2}+\left|\mu_{\mathrm{b}}\right|^{2}\right)^{1 / 2} \tau_{\mathrm{b}}+x^{\delta} f_{3}^{\sharp}\right) \phi_{\epsilon}\left(\tilde{\rho}^{-1}\right) .
\end{aligned}
$$

Correspondingly

$$
\begin{aligned}
& \mathrm{H}_{p} a_{\epsilon}=\left(\mathrm{H}_{p} a\right) \phi_{\epsilon}+a\left(\mathrm{H}_{p} \phi_{\epsilon}\left(\tilde{\rho}^{-1}\right)\right) \\
& \begin{aligned}
&=-2 x^{-l+1} \psi(x) \phi_{\epsilon}\left(\tilde{\rho}^{-1}\right)\left(( \pm \beta)\left(\tau_{\mathrm{b}}^{2}+\left|\mu_{\mathrm{b}}\right|^{2}\right)^{(\tilde{r}-3 / 2) / 2}\left(\tau_{\mathrm{b}}^{2}+x^{\delta} f_{1}^{\sharp}+\left(\left|\mu_{\mathrm{b}}\right|^{2}+x^{\delta} f_{2}^{\sharp}\right) \log \left(\tau_{\mathrm{b}}^{2}+\left|\mu_{\mathrm{b}}\right|^{2}\right)\right)\right. \\
&\left.\quad+\left(\tau_{\mathrm{b}}^{2}+\left|\mu_{\mathrm{b}}\right|^{2}\right)^{(\tilde{r}-1 / 2) / 2} K \epsilon\left(1+\epsilon\left(\tau_{\mathrm{b}}^{2}+\left|\mu_{\mathrm{b}}\right|^{2}\right)^{1 / 2}\right)^{-1}\left(\left(\tau_{\mathrm{b}}^{2}+\left|\mu_{\mathrm{b}}\right|^{2}\right)^{1 / 2} \tau_{\mathrm{b}}+x^{\delta} f_{3}^{\sharp}\right)\right) \\
& \quad+e \phi_{\epsilon}\left(\tilde{\rho}^{-1}\right) \\
&=-2 x^{-l+1} \psi(x) \phi_{\epsilon}\left(\tilde{\rho}^{-1}\right)\left(\tau_{\mathrm{b}}^{2}+\left|\mu_{\mathrm{b}}\right|^{2}\right)^{(\tilde{r}-3 / 2) / 2}\left(( \pm \beta)\left(\tau_{\mathrm{b}}^{2}+x^{\delta} f_{1}^{\sharp}+\left(\left|\mu_{\mathrm{b}}\right|^{2}+x^{\delta} f_{2}^{\sharp}\right) \log \left(\tau_{\mathrm{b}}^{2}+\left|\mu_{\mathrm{b}}\right|^{2}\right)\right)\right. \\
&\left.\quad+K\left(\tau_{\mathrm{b}}\left(\tau_{\mathrm{b}}^{2}+\left|\mu_{\mathrm{b}}\right|^{2}\right)^{1 / 2}+x^{\delta} f_{3}^{\sharp}\right) \frac{\epsilon\left(\tau_{\mathrm{b}}^{2}+\left|\mu_{\mathrm{b}}\right|^{2}\right)^{1 / 2}}{1+\epsilon\left(\tau_{\mathrm{b}}^{2}+\left|\mu_{\mathrm{b}}\right|^{2}\right)^{1 / 2}}\right)+e \phi_{\epsilon}\left(\tilde{\rho}^{-1}\right) .
\end{aligned}
\end{aligned}
$$

We point out that

$$
\frac{\epsilon\left(\tau_{\mathrm{b}}^{2}+\left|\mu_{\mathrm{b}}\right|^{2}\right)^{1 / 2}}{1+\epsilon\left(\tau_{\mathrm{b}}^{2}+\left|\mu_{\mathrm{b}}\right|^{2}\right)^{1 / 2}}
$$

is uniformly bounded in symbols of order 0 (for $\epsilon \in[0,1]$ ), with its supremum bounded by 1 , and tends to 0 as $\epsilon \rightarrow 0$ in symbols of positive order.

Notice that in the expression after the last equality in (3.12), for the + sign in \pm , the two terms in the big parentheses have the same sign in $\tau_{\mathrm{b}}>0$ and opposite signs in $\tau_{\mathrm{b}}<0$ (for $x$ small, when the $x^{\delta}$ terms can be absorbed in the others, as in the above discussion around (3.9) ), while for the $-\operatorname{sign}$ in \pm the roles of $\tau_{\mathrm{b}}>0$ and $\tau_{\mathrm{b}}<0$ reverse. Since for sufficiently large $\tau_{\mathrm{b}}^{2}+\left|\mu_{\mathrm{b}}\right|^{2}, \log \left(\tau_{\mathrm{b}}^{2}+\left|\mu_{\mathrm{b}}\right|^{2}\right)$ is bounded below by any pre-specified constant $C_{0}$, we have that $\tau_{\mathrm{b}}^{2}+\left|\mu_{\mathrm{b}}\right|^{2} \log \left(\tau_{\mathrm{b}}^{2}+\left|\mu_{\mathrm{b}}\right|^{2}\right) \geq$ $\tau_{\mathrm{b}}^{2}+C_{0}\left|\mu_{\mathrm{b}}\right|^{2}$ there. Correspondingly, for any $C_{1}>0$, in the region where in addition $\left|\tau_{\mathrm{b}}\right| \leq C_{1}\left|\mu_{\mathrm{b}}\right|, \tau_{\mathrm{b}}\left(\tau_{\mathrm{b}}^{2}+\left|\mu_{\mathrm{b}}\right|^{2}\right)^{1 / 2} \leq \tau_{\mathrm{b}}^{2}+\frac{1}{2}\left|\mu_{\mathrm{b}}\right|^{2} \leq\left(C_{1}^{2}+1\right)\left|\mu_{\mathrm{b}}\right|^{2}$ shows that in this region, the second term can be absorbed into, say, $\frac{1}{2}$ of the first term by choosing $C_{0}$ large. Taking the + sign in \pm for definiteness, in the complement of this region either $\tau_{\mathrm{b}}>0$, and thus the two terms have the same sign, or $\tau_{\mathrm{b}}<0$ and $\left|\mu_{\mathrm{b}}\right|<C_{1}^{-1}\left|\tau_{\mathrm{b}}\right|$; in this region thus the second term is bounded by $K\left(1+C_{1}^{-2}\right) \tau_{\mathrm{b}}^{2}$. Here $C_{1}>0$ can be chosen arbitrarily large, thus as $K<\beta$, the second term can be absorbed into the first. This is exactly the limit of regularization one can do, i.e. this step is the cause of the $K<\beta$ restriction in the statement of the proposition. Correspondingly, for the + sign in \pm (for the - sign, the overall - sign on the right hand side of the next equation is removed),

$$
2 a_{\epsilon} \mathrm{H}_{p} a_{\epsilon}=-\phi_{\epsilon}\left(\tilde{\rho}^{-1}\right)^{2}\left(b^{2}+b_{1}^{2}+b_{2, \epsilon}^{2}\right)+\phi_{\epsilon}\left(\tilde{\rho}^{-1}\right)^{2} a e
$$

with

$$
b^{2}=x^{-2 l} \psi(x)^{2}(\beta-K)\left(\tau_{\mathrm{b}}^{2}+\left|\mu_{\mathrm{b}}\right|^{2}\right)^{(\tilde{r}-1 / 2) / 2+(\tilde{r}-3 / 2) / 2+1},
$$

thus

$$
b=x^{-l} \sqrt{\beta-K} \psi(x)\left(\tau_{\mathrm{b}}^{2}+\left|\mu_{\mathrm{b}}\right|^{2}\right)^{\tilde{r}},
$$

and appropriate choices of $b_{1}$ and $b_{2, \epsilon}$. 
Let $B, B_{1}, B_{2, \epsilon}, S_{\epsilon}, E_{\epsilon}$ have principal symbols $b, b_{1}, b_{2, \epsilon}, \phi_{\epsilon}, \phi_{\epsilon}\left(\tilde{\rho}^{-1}\right)^{2} a e$ respectively, and so that $S_{\epsilon} \in \Psi_{\mathrm{b}}^{-K, 0}(X)$ uniformly bounded in $\Psi_{\mathrm{b}}^{0,0}(X)$, converging to Id in $\Psi_{\mathrm{b}}^{\delta^{\prime}, 0}(X)$ (with $\delta^{\prime}>0$ arbitrary), $B, B_{2, \epsilon} \in \Psi_{\mathrm{b}}^{\tilde{r}, l}(X)$, with $B_{2, \epsilon}$ uniformly bounded in this space, $B_{1} \in \Psi_{\mathrm{b}}^{\tilde{r}+\delta^{\prime}, l}(X)$ for all $\delta^{\prime}>0$ (arising from the variable order), while $E_{\epsilon}$ is uniformly bounded in $\Psi_{\mathrm{b}}^{2 \tilde{r}, 2 l}(X)$ and supported away from $\partial X$. Therefore, as can be seen by computing the principal symbol of both sides, which agree, one then has

$$
i\left(P(\sigma)^{*} A_{\epsilon}^{*} A_{\epsilon}-A_{\epsilon}^{*} A_{\epsilon} P(\sigma)\right)=-S_{\epsilon}^{*}\left(B^{*} B+B_{1}^{*} B_{1}+B_{2, \epsilon}^{*} B_{2, \epsilon}\right) S_{\epsilon}+E_{\epsilon}+F_{\epsilon},
$$

with $F_{\epsilon}$ uniformly bounded in $\Psi_{\mathrm{b}}^{2 \tilde{r}-1+\delta^{\prime}, 2 l}(X)$ for all $\delta^{\prime}>0$. Then

$$
\left\langle i\left(P(\sigma)^{*} A_{\epsilon}^{*} A_{\epsilon}-A_{\epsilon}^{*} A_{\epsilon} P(\sigma)\right) u, u\right\rangle=-\left\|B S_{\epsilon} u\right\|^{2}-\left\|B_{2, \epsilon} S_{\epsilon} u\right\|^{2}+\left\langle E_{\epsilon} u, u\right\rangle+\left\langle F_{\epsilon} u, u\right\rangle .
$$

Now,

$$
\left\langle i\left(P(\sigma)^{*} A_{\epsilon}^{*} A_{\epsilon}-A_{\epsilon}^{*} A_{\epsilon} P(\sigma)\right) u, u\right\rangle=\left\langle i A_{\epsilon} u, A_{\epsilon} P(\sigma) u\right\rangle-\left\langle i A_{\epsilon} P(\sigma) u, A_{\epsilon} u\right\rangle,
$$

where the moving of the adjoint over to the other side of the pairing actually requires an additional, straightforward, regularization (without the limitations on the amount of regularization, as in the case of $K$ above), see the proof of the radial point estimates in [45, Section 5.4.7]. Using Cauchy-Schwartz (with an elliptic operator used to shift the orders in the two slots of the pairing), estimating the product by a sum of squares, with a small constant $\digamma^{-1}>0$ in front of the $A_{\epsilon} u$ term, and absorbing the $A_{\epsilon} u$, one deduces the regularized version of the estimate for $\epsilon>0$ :

$$
\left\|B S_{\epsilon} u\right\|^{2} \leq \digamma\left\|A_{\epsilon} P(\sigma) u\right\|_{H_{\mathrm{b}}^{-1 / 2,1}(X)}^{2}+\left\langle E_{\epsilon} u, u\right\rangle+\left\langle F_{\epsilon} u, u\right\rangle .
$$

Let $\tilde{\psi}=\tilde{\psi}(x)$ be a $\mathcal{C}^{\infty}$ function, $\equiv 1$ on $\operatorname{supp} \psi$ still supported in a small collar neighborhood of $\partial X$. Now, for $u \in H_{\mathrm{b}}^{\tilde{r}-1 / 2+\delta^{\prime}, l}$ with $P(\sigma) \in H_{\mathrm{b}}^{\tilde{r}-1, l+2}$ all terms on the right hand side remain uniformly bounded as $\epsilon \rightarrow 0$ by the a priori assumptions, and by elliptic estimates in $x>0$, namely the latter gives, with a uniform constant,

$$
\left|\left\langle E_{\epsilon} u, u\right\rangle\right| \leq C^{\prime}\|\tilde{\psi} P(\sigma) u\|_{H_{\mathrm{b}}^{\tilde{r}-2, l^{\prime}}}^{2}+\|u\|_{H_{\mathrm{b}}^{\tilde{r}^{\prime}, l^{\prime}}}^{2}
$$

for any $\tilde{r}^{\prime}, \tilde{l}^{\prime}$. Thus, letting $\epsilon \rightarrow 0$, using the (sequential!) weak-* compactness of the unit ball in $L^{2}$ (so $B S_{\epsilon} u$ subsequentially converges) as well as that $B S_{\epsilon} u \rightarrow B u$ in tempered distributions, proves that for $0<K^{\prime}<\min (K, 1 / 2)$ and $\delta^{\prime}>0$ there exists $C^{\prime \prime}>0$ such that for $u \in H_{\mathrm{b}}^{\tilde{r}-K^{\prime}, l}$ with $P(\sigma) u \in H_{\mathrm{b}}^{\tilde{r}-1, l+2}$ we have

$$
\|\psi u\|_{H_{\mathrm{b}}^{\tilde{r}, l}} \leq C^{\prime \prime}\left(\|\tilde{\psi} P(\sigma) u\|_{H_{\mathrm{b}}^{\tilde{r}-1, l+2}}+\|u\|_{\left.H_{\mathrm{b}}^{\tilde{r}-1 / 2+\delta^{\prime}, l}\right)}\right)
$$

Then an iterative argument, of step size $<1 / 2$, improves $\tilde{r}-K^{\prime}$ in the norm on the right hand side to any $\tilde{r}^{\prime}$, under the assumption $u \in H_{\mathrm{b}}^{\tilde{r}-K, l}$.

Finally, as $P(\sigma)$ is elliptic away from $\partial X$, for any $\chi$ compactly supported in $X^{\circ}$ and $\tilde{\chi}$ also compactly supported there but $\equiv 1$ on $\operatorname{supp} \chi$, one has the elliptic estimate

$$
\|\chi u\|_{H_{\mathrm{b}}^{\tilde{r}, l}} \leq C^{\prime}\left(\|\tilde{\chi} P(\sigma) u\|_{H_{\mathrm{b}}^{\tilde{r}-1, l+2}}+\|u\|_{H_{\mathrm{b}}^{\tilde{r}-K, l}}\right)
$$

where the decay order actually does not matter (as all supports are away from $\partial X$, except for the last error term - but even that could be further localized). 
In combination, for any $K<\beta$ there exists $C>0$ such that for $u \in H_{\mathrm{b}}^{\tilde{r}-K, l}$ with $P(\sigma) u \in H_{\mathrm{b}}^{\tilde{r}-1, l+2}$ we have

$$
\|u\|_{H_{\mathrm{b}}^{\tilde{r}, l}} \leq C\left(\|P(\sigma) u\|_{H_{\mathrm{b}}^{\tilde{\mathrm{r}}-1, l+2}}+\|u\|_{H_{\mathrm{b}}^{\tilde{r}}-K, l}\right) .
$$

This thus proves (3.1).

Finally, if we allow $\sigma$ complex, with $\operatorname{Im} \sigma^{2}$ of the correct sign, matching \pm in (1.6), then in (3.3), we have the extra term $i\left(P(\sigma)^{*}-P(\sigma)\right) A^{*} A$. We only consider the case of the spectral family, when this is $i\left(\sigma^{2}-\bar{\sigma}^{2}\right) a^{2}=-2 \operatorname{Im}\left(\sigma^{2}\right) a^{2}$, thus matches the sign of $2 a \mathrm{H}_{p} a$ in the $\operatorname{Im}\left(\sigma^{2}\right) \geq 0,+\beta$ choice for $\tilde{r}$ case, as well as in the $\operatorname{Im}\left(\sigma^{2}\right) \leq 0,-\beta$ choice for $\tilde{r}$ case, thus can simply be dropped from the estimate.

Proposition 3.1 has the following strengthened more geometric version:

Proposition 3.5. Suppose that $\tilde{r}$ is a homogeneous degree 0 function on ${ }^{\mathrm{b}} T^{*} X \backslash o$ (i.e. a smooth function on $\left.{ }^{\mathrm{b}} S^{*} X\right)$ with $\tilde{r}>\frac{1}{2}-(l+1)$ at the source, $\left\{\tau_{\mathrm{b}}>0, \mu_{\mathrm{b}}=0\right\}$, $\tilde{r}<\frac{1}{2}-(l+1)$ at the sink, $\left\{\tau_{\mathrm{b}}<0, \mu_{\mathrm{b}}=0\right\}$, and $-\left(\tau_{\mathrm{b}}^{2}+\left|\mu_{\mathrm{b}}\right|^{2}\right)^{-1 / 2} x^{-2} \mathrm{H}_{p_{\partial}} \tilde{r}$ is non-negative. Let $K>0$ be such that $\tilde{r}>\frac{1}{2}-(l+1)+K$ in a neighborhood of the source.

For $\sigma$ in a compact subset of $[0, \infty)$ there exists $C>0$ such that for all $u \in$ $H_{\mathrm{b}}^{\tilde{r}-K, l}$ with $P(\sigma) u \in H_{\mathrm{b}}^{\tilde{r}-1, l+2}$, we have $u \in H_{\mathrm{b}}^{\tilde{r}, l}$ and

$$
\|u\|_{H_{\mathrm{b}}^{\tilde{r}, l}} \leq C\left(\|P(\sigma) u\|_{H_{\mathrm{b}}^{\tilde{r}-1, l+2}}+\|u\|_{H_{\mathrm{b}}^{\tilde{r}-K, l}}\right) .
$$

The analogous result also holds with the source and sink switched, and the positivity of $-\left(\tau_{\mathrm{b}}^{2}+\left|\mu_{\mathrm{b}}\right|^{2}\right)^{-1 / 2} x^{-2} \mathrm{H}_{p_{\partial}} \tilde{r}$ is replaced by that of $\left(\tau_{\mathrm{b}}^{2}+\left|\mu_{\mathrm{b}}\right|^{2}\right)^{-1 / 2} x^{-2} \mathrm{H}_{p_{\partial}} \tilde{r}$.

Remark 3.6. Note that the + sign in $\pm \beta$ in Proposition 3.1 corresponds to the first case of this proposition, while the - sign corresponds to the second case ('analogous result').

Proof. We can follow the proof of Proposition 3.1 very closely, however we modify the commutant slightly and consider, for $\tilde{\beta}>0$ to be specified and with \pm meaning + for the first case of the proposition, - for the second ('analogous') case,

$$
a=x^{-l-1}\left(\tau_{\mathrm{b}}^{2}+\left|\mu_{\mathrm{b}}\right|^{2}\right)^{(\tilde{r}-1 / 2) / 2} \psi(x) \exp \left( \pm \frac{\tilde{\beta}}{2} \frac{\tau_{\mathrm{b}}}{\left(\tau_{\mathrm{b}}^{2}+\left|\mu_{\mathrm{b}}\right|^{2}\right)^{1 / 2}}\right),
$$

which is

$$
\exp \left( \pm \frac{\tilde{\beta}}{2} \frac{\tau_{\mathrm{b}}}{\left(\tau_{\mathrm{b}}^{2}+\left|\mu_{\mathrm{b}}\right|^{2}\right)^{1 / 2}}\right)
$$

times the principal symbol used for our symbolic computation in (3.4), except that our choice of $\tilde{r}$ has been generalized from (3.5). Notice that this new factor is a smooth function on ${ }^{\mathrm{b}} S^{*} X$, thus does not change the order of $a$, but it does affect the principal symbol. Its role is to obtain positivity of the commutator even away from the radial points even when $\tilde{r}$ has an indefinite derivative along $H_{p_{\partial}}$; indeed note that this is the exponential of the non-constant part of the choice of $\tilde{r}$ employed in (3.5). We remark that (3.16) will be the principal symbol of our commutant choice for the normal operator computation in 4.24) (albeit with the special choice of $\tilde{r}$ from (3.5); we added the $\frac{1}{2}$ factor in front of $\beta$ for consistency of notation with the next section. 
Below we prove the first case of the proposition; the analogous case is completely similar. Thus, we take the sign in front of $\tilde{\beta}$ to be positive. In (3.6), there is now a new overall factor 3.17 on the first line, the third line is replaced by

$$
+\frac{1}{2} x^{-2}\left(\mathrm{H}_{p_{\partial}} \tilde{r}\right)\left(\tau_{\mathrm{b}}^{2}+\left|\mu_{\mathrm{b}}\right|^{2}\right)^{(\tilde{r}-1 / 2) / 2} \log \left(\tau_{\mathrm{b}}^{2}+\left|\mu_{\mathrm{b}}\right|^{2}\right) \psi(x),
$$

and there is a new line, inserted between the third and fourth:

$$
-\left(\tau_{\mathrm{b}}^{2}+\left|\mu_{\mathrm{b}}\right|^{2}\right)^{1+(\tilde{r}-1 / 2) / 2} \frac{\tilde{\beta}}{2} \partial_{\tau_{\mathrm{b}}}\left(\frac{\tau_{\mathrm{b}}}{\left(\tau_{\mathrm{b}}^{2}+\left|\mu_{\mathrm{b}}\right|^{2}\right)^{1 / 2}}\right) \psi(x),
$$

which is

$$
-\frac{\tilde{\beta}}{2}\left|\mu_{\mathrm{b}}\right|^{2}\left(\tau_{\mathrm{b}}^{2}+\left|\mu_{\mathrm{b}}\right|^{2}\right)^{-1 / 2+(\tilde{r}-1 / 2) / 2} \psi(x),
$$

cf. (3.7).

Thus 3.8 is replaced by

$$
\begin{gathered}
\mathrm{H}_{p} a=-2 x^{-l+1} \psi(x)\left(\tau_{\mathrm{b}}^{2}+\left|\mu_{\mathrm{b}}\right|^{2}\right)^{(\tilde{r}-3 / 2) / 2} \\
\cdot\left(\left(\left(\tilde{r}-\frac{1}{2}+(l+1)\right) \tau_{\mathrm{b}}+\frac{\tilde{\beta}}{2}\left|\mu_{\mathrm{b}}\right|^{2}+x^{\delta} f_{1}^{\sharp}\right)\left(\tau_{\mathrm{b}}^{2}+\left|\mu_{\mathrm{b}}\right|^{2}\right)^{1 / 2}\right. \\
\left.+\left(-\frac{1}{2}\left(\tau_{\mathrm{b}}^{2}+\left|\mu_{\mathrm{b}}\right|^{2}\right)^{1 / 2} x^{-2} \mathrm{H}_{p_{\partial}} \tilde{r}+x^{\delta} f_{2}^{\sharp}\right) \log \left(\tau_{\mathrm{b}}^{2}+\left|\mu_{\mathrm{b}}\right|^{2}\right)\right)+e, \\
e=2 x^{2} x^{-l-1} x \psi^{\prime}(x)\left(\tau_{\mathrm{b}}+x^{\delta} f_{0}^{\sharp}\right)\left(\tau_{\mathrm{b}}^{2}+\left|\mu_{\mathrm{b}}\right|^{2}\right)^{(\tilde{r}-1 / 2) / 2}
\end{gathered}
$$

with $f_{0}^{\sharp}$ homogeneous of degree $1, f_{1}^{\sharp}, f_{2}^{\sharp}$ homogeneous of degree 2 . Now

$$
\left(\tilde{r}-\frac{1}{2}+(l+1)\right) \tau_{\mathrm{b}}\left(\tau_{\mathrm{b}}^{2}+\left|\mu_{\mathrm{b}}\right|^{2}\right)^{1 / 2}
$$

is bounded below by a positive multiple of $\tau_{\mathrm{b}}^{2}+\left|\mu_{\mathrm{b}}\right|^{2}$ near the sources and sinks, while $\frac{\tilde{\beta}}{2}\left|\mu_{\mathrm{b}}\right|^{2}$ is similarly bounded below on any compact set disjoint from the sources and sinks, such as on the complement of a set on which the first term had the desired positive bound, and is nonnegative everywhere. Correspondingly, choosing $\tilde{\beta}>0$ sufficiently large, the sum of these two terms is bounded below by a positive multiple of $\tau_{\mathrm{b}}^{2}+\left|\mu_{\mathrm{b}}\right|^{2}$. Also, $-\frac{1}{2}\left(\tau_{\mathrm{b}}^{2}+\left|\mu_{\mathrm{b}}\right|^{2}\right)^{1 / 2} x^{-2} \mathrm{H}_{p_{\partial}} \tilde{r}$ is non-negative. One can then complete the argument as in the proof of Proposition 3.1 when one has $u \in$ $H_{\mathrm{b}}^{\tilde{r}, l}$ a priori (thus there is no need for regularization). In the general case, the regularization again proceeds almost as before since the regularization factor is unchanged, and now increasing $\tilde{\beta}$ can dominates this regularization factor away from the sources and sinks, while near the source the two terms have the same sign, while near the sink, $\left|\mu_{\mathrm{b}}\right|<C_{1}^{-1}\left|\tau_{\mathrm{b}}\right|$ where $C_{1}>0$ can be chosen large; in this region thus the second, regularization, term of the big parentheses of 3.12 is bounded by $K\left(1+C_{1}^{-2}\right) \tau_{\mathrm{b}}^{2}$, and can be absorbed into the

$$
\left.\left(\tilde{r}-\frac{1}{2}+(l+1)\right) \tau_{\mathrm{b}}+\frac{\tilde{\beta}}{2}\left|\mu_{\mathrm{b}}\right|^{2}+x^{\delta} f_{1}^{\sharp}\right)\left(\tau_{\mathrm{b}}^{2}+\left|\mu_{\mathrm{b}}\right|^{2}\right)^{1 / 2}
$$

term of 3.18 as $\tilde{r}-\frac{1}{2}+(l+1)-K>0$. This completes the proof in general. 


\section{THE NORMAL OPERATOR AND PROOF OF THE MAIN THEOREM}

\subsection{Proof of the main theorem from an estimate from a normal operator} estimate. We now improve on (3.1) and 3.15),

$$
\|u\|_{H_{\mathrm{b}}^{\tilde{r}, l}} \leq C\left(\|P(\sigma) u\|_{H_{\mathrm{b}}^{\tilde{r}-1, l+2}}+\|u\|_{\left.H_{\mathrm{b}}^{\tilde{r}-K, l}\right)},\right.
$$

valid for $u \in H_{\mathrm{b}}^{\tilde{r}-K, l}, K>0$ with $\tilde{r}>\frac{1}{2}-(l+1)+K$ at the source in case $\tilde{r}$ is monotone decreasing in the sense of Proposition 3.5. with $P(\sigma) u \in H_{\mathrm{b}}^{\tilde{r}-1, l+2}$, and an analogous statement with source replaced by sink if $\tilde{r}$ is monotone increasing. Recall from Remark 3.4 that in fact $\|u\|_{H_{\mathrm{b}}^{\tilde{r}-K, l}}$ can be replaced by $\|u\|_{H_{\mathrm{b}}^{-N, l}}$ for any $-N$, as long as one keeps in mind that one needs to have $u \in H_{\mathrm{b}}^{\tilde{r}-K, l}, K>0$ as above, a priori.

Namely, we make the error term on the right hand side, $\|u\|_{H_{\mathrm{b}}^{\tilde{r}-K, l}}$, replaced by a compact error. Directly we prove:

Proposition 4.1. Let $S \subset[0, \infty)$ compact, and suppose that $\tilde{r}$ is as in Proposition 3.5: $\tilde{r}$ is a homogeneous degree 0 function on ${ }^{\mathrm{b}} T^{*} X \backslash$ o (i.e. a smooth function on $\left.{ }^{\mathrm{b}} S^{*} X\right)$ with $\tilde{r}>\frac{1}{2}-(l+1)$ at the source, $\left\{\tau_{\mathrm{b}}>0, \mu_{\mathrm{b}}=0\right\}, \tilde{r}<\frac{1}{2}-(l+1)$ at the sink, $\left\{\tau_{\mathrm{b}}<0, \mu_{\mathrm{b}}=0\right\}$, and $-\left(\tau_{\mathrm{b}}^{2}+\left|\mu_{\mathrm{b}}\right|^{2}\right)^{-1 / 2} x^{-2} \mathrm{H}_{p_{\partial}} \tilde{r}$ is non-negative. Let $K>0$ be such that $\tilde{r}>\frac{1}{2}-(l+1)+K$ in a neighborhood of the source.

There exists $C$ such that for $u \in H_{\mathrm{b}}^{\tilde{r}-K, l}$ with $P(\sigma) u \in H_{\mathrm{b}}^{\tilde{r}-1, l+2}$, the estimate

$$
\|u\|_{H_{\mathrm{b}}^{\tilde{r}, l}} \leq C\left(\|P(\sigma) u\|_{H_{\mathrm{b}}^{\tilde{r}-1, l+2}}+\|u\|_{H_{\mathrm{b}}^{\tilde{r}-K, l-\delta}}\right)
$$

holds for $\sigma \in S$.

The analogous result also holds with the source and sink switched, and the positivity of $-\left(\tau_{\mathrm{b}}^{2}+\left|\mu_{\mathrm{b}}\right|^{2}\right)^{-1 / 2} x^{-2} \mathrm{H}_{p_{\partial}} \tilde{r}$ is replaced by that of $\left(\tau_{\mathrm{b}}^{2}+\left|\mu_{\mathrm{b}}\right|^{2}\right)^{-1 / 2} x^{-2} \mathrm{H}_{p_{\partial}} \tilde{r}$.

Remark 4.2. As an example, $\tilde{r}=\hat{r}_{ \pm}$satisfies the requirements where

$$
\hat{r}_{ \pm}=\hat{r}_{ \pm}(\beta)=\frac{1}{2}-(l+1) \pm \beta \frac{\tau_{\mathrm{b}}}{\left(\tau_{\mathrm{b}}^{2}+\left|\mu_{\mathrm{b}}\right|^{2}\right)^{1 / 2}}, \quad \beta>0 ;
$$

the + sign corresponds to the first case, the - sign to the second ('analogous') case.

Remark 4.3. The improvement in the differential order of the Sobolev norm of $u$ on the right hand side is actually arbitrary, i.e. $\tilde{r}-K$ could be replaced by $-N, N$ arbitrary, with the understanding that one needs membership of $u$ in $H_{\mathrm{b}}^{\tilde{r}-K, l}$ for some $0<K<\beta$, cf. Remark 3.4. In fact, by similar considerations the decay order is also arbitrary, but we still need the $H_{\mathrm{b}}^{\tilde{r}-K, l}$ membership of $u$ as stated.

A standard argument allows one to conclude from this a uniform estimate without a compact error under an injectivity hypothesis; note that this immediately implies Theorem 1.1, which we restate in the present stronger version below.

Proposition 4.4. With the notation of Proposition 4.1. suppose that $P(0): H_{\mathrm{b}}^{\tilde{r}, l} \rightarrow$ $H_{\mathrm{b}}^{\tilde{r}-2, l+2}$ has trivial nullspace. There exist $\sigma_{0}>0$ and $C^{\prime}>0$ such that for $|\sigma|<\sigma_{0}$,

$$
\|u\|_{H_{\mathrm{b}}^{\tilde{r}, l}} \leq C^{\prime}\|P(\sigma) u\|_{H_{\mathrm{b}}^{\tilde{r}-1, l+2}}
$$

for $u \in H_{\mathrm{b}}^{\tilde{r}, l}$ with $P(\sigma) u \in H_{\mathrm{b}}^{\tilde{r}-1, l+2}$. 
Proof of Proposition 4.4 given Proposition 4.1. Indeed, if 4.4 is not true, there is a sequence $u_{j}$, which one may assume has unit norm in $H_{\mathrm{b},}^{r, l}$, and with $P\left(\sigma_{j}\right) u_{j} \in$ $H_{\mathrm{b}}^{\tilde{r}-1, l+2}$, and a sequence $\sigma_{j} \rightarrow 0$ such that $P\left(\sigma_{j}\right) u_{j} \rightarrow 0$ in $H_{\mathrm{b}}^{\tilde{r}-1, l+2}$. By taking a subsequence (not shown in notation), using the sequential compactness of the unit ball in $H_{\mathrm{b}}^{\tilde{r}, l}$ in the weak topology, and the compactness of the inclusion $H_{\mathrm{b}}^{\tilde{r}, l} \rightarrow$ $H_{\mathrm{b}}^{\tilde{r}-K, l-\delta}, K>0$, one may assume that there is $u \in H_{\mathrm{b}}^{\tilde{r}, l}$ such that $u_{j} \rightarrow u$ weakly in $H_{\mathrm{b}}^{\tilde{r}, l}$ and strongly in $H_{\mathrm{b}}^{\tilde{r}-K, l-\delta}$. By 4.2 then liminf $\left\|u_{j}\right\|_{H_{\mathrm{b}}^{\tilde{r}-K, l-\delta}} \geq C^{-1}>0$, so $u \neq 0$ by the strong convergence. On the other hand, $P\left(\sigma_{j}\right) u_{j} \rightarrow P(0) u$ in $H_{\mathrm{b}}^{\tilde{r}-K-2, l-\delta}$ as

$$
P\left(\sigma_{j}\right) u_{j}-P(0) u=\left(P\left(\sigma_{j}\right)-P(0)\right) u_{j}+P(0)\left(u_{j}-u\right)
$$

since $P\left(\sigma_{j}\right) \rightarrow P(0)$ as bounded operators in $\mathcal{L}\left(H_{\mathrm{b}}^{\tilde{r}-K, l-\delta}, H_{\mathrm{b}}^{\tilde{r}-K-2, l-\delta}\right)$ and $u_{j}$ converges to $u$ (thus is bounded) in $H_{\mathrm{b}}^{\tilde{r}-K, l-\delta}$. Thus, $P(0) u=0$, so $u$ is a nontrivial element of the nullspace of $P(0)$ on $H_{\mathrm{b}}^{\tilde{r}, l}$, which contradicts our assumptions. This proves (4.4), and thus the proposition.

Since the nullspace of (the elliptic in $\left.x^{2} \operatorname{Diff}_{\mathrm{b}}^{2}(X) !\right) P(0)$ is independent of the differential order $\tilde{r}$, this immediately implies our main theorem, which we now state in a slightly strengthened version:

Theorem 4.5. Suppose that $\tilde{r}$ is as in Proposition 3.5: $\tilde{r}$ is a homogeneous degree 0 function on ${ }^{\mathrm{b}} T^{*} X \backslash$ o (i.e. a smooth function on $\left.{ }^{\mathrm{b}} S^{*} X\right)$ with $\tilde{r}>\frac{1}{2}-(l+1)$ at the source, $\left\{\tau_{\mathrm{b}}>0, \mu_{\mathrm{b}}=0\right\}, \tilde{r}<\frac{1}{2}-(l+1)$ at the sink, $\left\{\tau_{\mathrm{b}}<0, \mu_{\mathrm{b}}=0\right\}$, and $-\left(\tau_{\mathrm{b}}^{2}+\left|\mu_{\mathrm{b}}\right|^{2}\right)^{-1 / 2} x^{-2} \mathrm{H}_{p_{\partial}} \tilde{r}$ is non-negative.

Suppose also that $|l+1|<\frac{n-2}{2}$ and $P(0): H_{\mathrm{b}}^{\infty, l} \rightarrow H_{\mathrm{b}}^{\infty, l+2}$ has trivial nullspace.

Then there exist $\sigma_{0}>0$ and $C>0$ such that for $|\sigma|<\sigma_{0}, \operatorname{Im}\left(\sigma^{2}\right) \geq 0$,

$$
\left\|P(\sigma)^{-1} f\right\|_{H_{\mathrm{b}}^{\tilde{r}, l}} \leq C\|f\|_{H_{\mathrm{b}}^{\tilde{r}-1, l+2}}
$$

for $f \in H_{\mathrm{b}}^{\tilde{r}-1, l+2}$.

The analogous result also holds with the source and sink switched, and the positivity of $-\left(\tau_{\mathrm{b}}^{2}+\left|\mu_{\mathrm{b}}\right|^{2}\right)^{-1 / 2} x^{-2} \mathrm{H}_{p_{\partial}} \tilde{r}$ is replaced by that of $\left(\tau_{\mathrm{b}}^{2}+\left|\mu_{\mathrm{b}}\right|^{2}\right)^{-1 / 2} x^{-2} \mathrm{H}_{p_{\partial}} \tilde{r}$ provided one also changes the condition on $\sigma^{2}$ to $\operatorname{Im}\left(\sigma^{2}\right) \leq 0$.

Thus, it suffices to prove Proposition 4.1, i.e. 4.2, which we means we need to gain decay for the error term of 4.1) on the right hand side.

In general, decay is controlled by the normal operator of a b-differential operator, which arises by setting $x=0$ in its coefficients after factoring out an overall weight, and where one thinks of it as acting on functions on $[0, \infty)_{x} \times \partial X$, of which $\left[0, \delta_{0}\right)_{x} \times$ $\partial X$ is identified with a neighborhood of $\partial X$ in $X$. Now, $P(\sigma) \in \Psi_{\mathrm{b}}^{-2,0}$ only, and in the usual sense the normal operator is simply $-\sigma^{2}$ as $P(\sigma)+\sigma^{2} \in \Psi_{\mathrm{b}}^{-2,2}$. Thus, we instead consider the 'effective normal operator', which from 1.2 , namely

$$
P(\sigma)=P(0)+\sigma Q-\sigma^{2}, \quad P(0) \in x^{2} \operatorname{Diff}_{\mathrm{b}}^{2}(X), Q \in x^{3} \operatorname{Diff}_{\mathrm{b}}^{1}(X)
$$

is

$$
\tilde{N}(P(\sigma))=N(P(0))-\sigma^{2},
$$

so

$$
P(\sigma)-\tilde{N}(P(\sigma)) \in x^{2+\delta} S^{0} \operatorname{Diff}_{\mathrm{b}}^{2}(X) .
$$


For a normally long-range asymptotically Euclidean metric, we have

$$
\tilde{N}(P(\sigma))=\Delta_{\text {sc }}-\sigma^{2}, \text { where } \Delta_{\text {sc }}=x^{n+1} D_{x} x^{-n-1} x^{4} D_{x}+x^{2} \Delta_{\partial X}
$$

is the model scattering Laplacian at infinity.

Typically in b-problems one proceeds by obtaining separate principal symbol and normal operator estimates. In the present case, in Section 4.2, we prove a normal operator estimate with the special case of the differential order $\hat{r}=\hat{r}_{ \pm}(\beta)$ :

Proposition 4.6. For $S \subset[0, \infty)$ compact, the effective normal operator $\tilde{N}(P(\sigma))$ satisfies

$$
\|v\|_{H_{\mathrm{b}}^{\hat{r}, l}} \leq C\|\tilde{N}(P(\sigma)) v\|_{H_{\mathrm{b}}^{\hat{\mathrm{r}}-1, l+2}}
$$

with $\hat{r}=\hat{r}_{ \pm}(\beta), \beta>0$, as in 4.3 .

Due to the lack of ellipticity, namely the loss of one derivative in 4.1 on $P(\sigma) u$ relative to the elliptic shift of the order of the norm on the left hand side, this will give a somewhat weaker estimate than needed for Proposition 4.1 even in the special case $\tilde{r}=\hat{r}$, but we improve on it by using the symbolic estimate yet again. In the special case $\tilde{r}=\hat{r}$ this could be avoided by doing both the symbolic and normal operator positivity argument in a single step (rather than broken up into two steps, as done in the previous and the current sections), but as we intend to prove the general differential order $\tilde{r}$ version of the main theorem in Theorem 4.5 . yet prove (and use) the normal operator positivity only for the special differential order $\hat{r}$, we give a unified, but slightly involved, functional analytic argument below.

We now proceed to prove Proposition 4.1, given Proposition 4.6 .

Proof of Proposition 4.1 given Proposition 4.6. Suppose (4.7) holds.

We first show the estimate (4.2) of Proposition 4.1 under the additional assumption that $\tilde{r}>\hat{r}+1$, where $\hat{r}=\hat{r}_{+}(\beta)$ with $\beta$ fixed. Notice that this can only be satisfied, in view of the low regularity radial point estimate, if $\beta>1$, but in that case it indeed can be satisfied e.g. by $\tilde{r}=\hat{r}+1+\epsilon, 0<\epsilon<\beta-1$. We point out that under the 'analogous' second part of the statement of the proposition, the same arguments prove 4.2 under the additional assumption that $\tilde{r}>\hat{r}_{-}(\beta)+1$; this will be used later in duality arguments.

We start with 4.1):

$$
\|u\|_{H_{\mathrm{b}}^{\tilde{r}, l}} \leq C\left(\|P(\sigma) u\|_{H_{\mathrm{b}}^{\tilde{r}-1, l+2}}+\|u\|_{H_{\mathrm{b}}^{-N, l}}\right),
$$

where we may assume that $u \in \dot{\mathcal{C}}^{\infty}(X)$, so membership in various spaces below is automatic, and where we take $N>\beta$. It thus remains to estimate the error term $\|u\|_{H_{\mathrm{b}}^{-N, l} \text {. }}$

Let $\chi$ be a cutoff supported in a collar neighborhood of the boundary, identically 1 on a smaller neighborhood. Then

$$
\|u\|_{H_{\mathrm{b}}^{-N, l}} \leq\|\chi u\|_{H_{\mathrm{b}}^{-N, l}}+\|(1-\chi) u\|_{H_{\mathrm{b}}^{-N, l}}
$$

shows that it suffices to estimate the $\chi u$ term (for the other is compactly supported in the interior, so can be absorbed into the second term of the right hand side of (4.2)). This in turn is estimated, thanks to 4.7, by

$$
\|\chi u\|_{H_{\mathrm{b}}^{-N, l}} \leq\|\chi u\|_{H_{\mathrm{b}}^{\hat{\mathrm{r}}, l}} \leq C\|\hat{N}(P(\sigma))(\chi u)\|_{H_{\mathrm{b}}^{\hat{r}-1, l+2}} .
$$


Further (with $l^{\prime}$ arbitrary in the compactly supported term below)

$$
\begin{aligned}
& \|\tilde{N}(P(\sigma))(\chi u)\|_{H_{\mathrm{b}}^{\hat{r}-1, l+2}} \\
& \leq\|P(\sigma) u\|_{H_{\mathrm{b}}^{\hat{r}-1, l+2}}+\|P(\sigma)(1-\chi) u\|_{H_{\mathrm{b}}^{\hat{r}-1, l+2}}+\|(P(\sigma)-\tilde{N}(P(\sigma)))(\chi u)\|_{H_{\mathrm{b}}^{\hat{r}-1, l+2}} \\
& \leq\|P(\sigma) u\|_{H_{\mathrm{b}}^{\hat{r}-1, l+2}}+C^{\prime}\|u\|_{H_{\mathrm{b}}^{\hat{r}+1, l^{\prime}}}+C^{\prime}\|\chi u\|_{H_{\mathrm{b}}^{\hat{r}+1, l-\delta}} \\
& \leq\|P(\sigma) u\|_{H_{\mathrm{b}}^{\hat{r}-1, l+2}}+C\|u\|_{H_{\mathrm{b}}^{\hat{r}+1, l-\delta}}
\end{aligned}
$$

Thus,

$$
\|u\|_{H_{\mathrm{b}}^{\tilde{r}, l}} \leq C\left(\|P(\sigma) u\|_{H_{\mathrm{b}}^{\tilde{r}-1, l+2}}+\|P(\sigma) u\|_{H_{\mathrm{b}}^{\hat{r}-1, l+2}}+\|u\|_{\left.H_{\mathrm{b}}^{\hat{r}+1, l-\delta}\right)}\right)
$$

As $\hat{r}-1 \leq \tilde{r}-1$ and $\hat{r}+1<\tilde{r}$, this gives

$$
\begin{aligned}
\|u\|_{H_{\mathrm{b}}^{\tilde{r}}, l} & \leq C\left(\|P(\sigma) u\|_{H_{\mathrm{b}}^{\tilde{r}-1, l+2}}+\|u\|_{H_{\mathrm{b}}^{\hat{r}+1, l-\delta}}\right) \\
& \leq C\|P(\sigma) u\|_{H_{\mathrm{b}}^{\tilde{r}-1, l+2}}+\epsilon\|u\|_{H_{\mathrm{b}}^{\tilde{r}, l-\delta}}+C_{\epsilon}\|u\|_{H_{\mathrm{b}}^{\tilde{r}-K, l-\delta}},
\end{aligned}
$$

and now the second term on the right hand side can be absorbed into the left hand side to yield

$$
\|u\|_{H_{\mathrm{b}}^{\tilde{r}, l}} \leq C\left(\|P(\sigma) u\|_{H_{\mathrm{b}}^{\tilde{r}-1, l+2}}+\|u\|_{\left.H_{\mathrm{b}}^{\tilde{r}-K, l-\delta}\right)},\right.
$$

This proves the estimate (4.2) of Proposition 4.1 in the case of $\tilde{r}>\hat{r}+1$ (which, again, requires $\beta>1$ to be non-vacuous), completing our first goal.

As already mentioned at the beginning of the proof, this also proves the estimate 4.2 in the second part of Proposition 4.1 in the case of $\tilde{r}_{>} \hat{r}_{-}(\beta)+1$.

Before proceeding, we remark that using the regularity estimate 4.1), valid under just the assumption that its right hand side is finite (i.e. that $u$ and $P(\sigma) u$ are in the appropriate spaces), we in fact obtain that 4.9 holds if $P(\sigma) u \in H_{\mathrm{b}}^{\tilde{r}-1, l+2}$ and $u \in H_{\mathrm{b}}^{\tilde{r}-K, l}$. Notice that this is a stronger condition than the right hand side of 4.9 being finite; the reason is that while in Section 3 we did a regularization argument for the differential order, we do not perform a similar argument for the decay order in the present section.

Equation (4.9) is only one half of a Fredholm estimate: it implies closed range and a finite dimensional nullspace. To see the other half, which gives that the range is finite codimensional, we need to dualize and work with $P(\sigma)^{*}$. In order to keep the notation clear, write $\hat{r}_{ \pm}$corresponding to the sign \pm in the definition of $\hat{r}$, and similarly write $\tilde{r}_{ \pm}$with $\tilde{r}_{ \pm}>\hat{r}_{ \pm}+1$. Now, the dual of $H_{\mathrm{b}}^{\tilde{r}_{+}, l}$ is $H_{\mathrm{b}}^{-\tilde{r}_{+},-l}$ thus that of $H_{\mathrm{b}}^{\tilde{r}_{+}-1, l+2}$ is $H_{\mathrm{b}}^{1-\tilde{r}_{+},-l-2}$, so correspondingly we would like to have an estimate

$$
\|v\|_{H_{\mathrm{b}}^{1-\tilde{r}_{+},-l-2}} \leq C\left(\left\|P(\sigma)^{*} v\right\|_{H_{\mathrm{b}}^{-\tilde{r}_{+},-l}}+\|v\|_{H_{\mathrm{b}}^{1-\tilde{r}_{+}-K,-l-2-\delta}}\right)
$$

to complete the argument.

Our next goal is to prove 4.10 in the case $\tilde{r}_{+}>\hat{r}_{+}+1$. Notice that $-\tilde{r}_{+}=$ $\left(1-\tilde{r}_{+}\right)-1,-l=(-l-2)+2$, so the arithmetic relationship between the spaces on the left and right hand side of the estimate is the same as in 4.9 . Now, $-l-2$ satisfies the same assumptions as $l$, namely $|(-l-2)+1|<\frac{n-2}{2}$. Moreover,

$$
\tilde{r}_{+}>\frac{1}{2}-(l+1), \text { resp. } \tilde{r}_{+}<\frac{1}{2}-(l+1)
$$

microlocally at the source or sink are equivalent to

$$
1-\tilde{r}_{+}<\frac{1}{2}-(-(l+2)+1), \text { resp. } 1-\tilde{r}_{+}>\frac{1}{2}-(-(l+2)+1),
$$




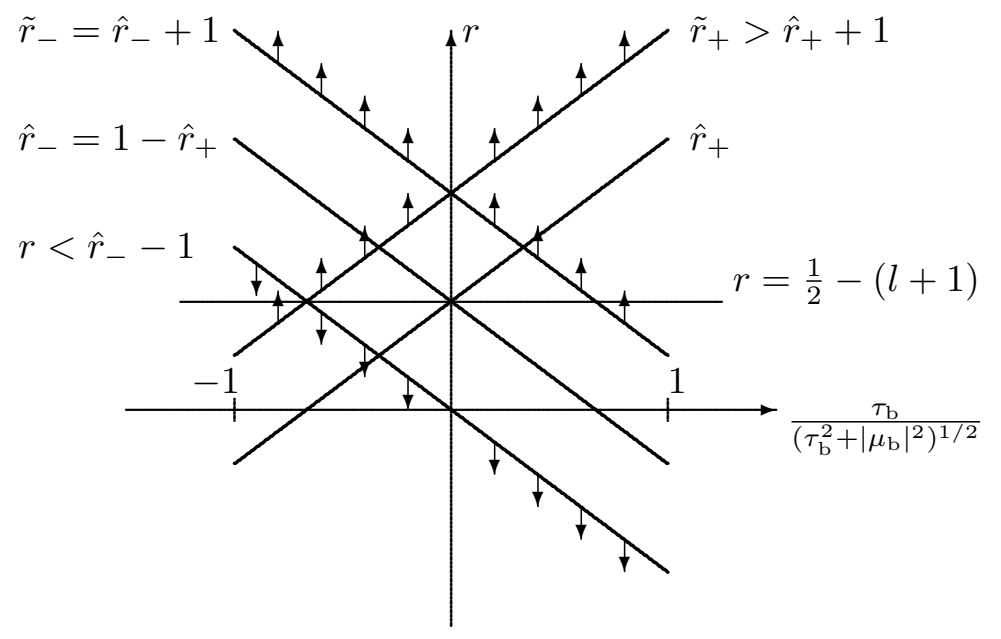

FiguRE 2. The variable order functions used in the proof: in order for the symbolic estimate (4.1) to apply one needs a weight $r$ which is monotone and is strictly below, resp. above, the critical line $r=$ $\frac{1}{2}-(l+1)$ at exactly one of -1 and 1 . (Of course, the symbolic order need not be a function of $\frac{\tau_{\mathrm{b}}}{\left(\tau_{\mathrm{b}}^{2}+\left|\mu_{\mathrm{b}}\right|^{2}\right)^{1 / 2}}$ only.) On the $\tilde{r}_{+}=\hat{r}_{+}+1$ line, the arrows indicate the region $\tilde{r}_{+}>\hat{r}_{+}+1$ in which the graph of $\tilde{r}_{+}$must lie for the first step of our proof to apply. Then $r<\hat{r}_{-}-1$ indicates the region where the graph of the order on the dual space must correspondingly lie to create a Fredholm estimate; this is however not admissible for our proof of $\sqrt{4.9}$ in this first step to hold. Finally, the line $\tilde{r}_{-}=\hat{r}_{-}+1$ shows the dual order that is actually used in the second step; the argument of the first step applies to this when replacing $P(\sigma)$ by $P(\sigma)^{*}$ and $\hat{r}_{+}$by $\hat{r}_{-}$ - the arrows indicate the region in which the graph of $\tilde{r}_{-}$must be for the argument of the first step to apply.

i.e. for $\tilde{r}_{+}, l$ satisfying the conditions for the symbolic estimates for the + choice of sign, the dual spaces of order $1-\tilde{r}_{+},-l-2$ satisfy the analogous conditions with the location of high and low regularity reversed. Thus, as $P(\sigma)^{*}$ satisfies the same assumptions as $P(\sigma), 4.10$ is indeed the same kind of estimate as 4.9$)$, but with monotonicity direction (increase/decrease) along the $H_{p}$ flow is reversed. The only issue, due to which 4.9) (with $P(\sigma)^{*}$ in place of $P(\sigma)$ ) does not immediately yield 4.10), is that in 4.9) (now with $P(\sigma)^{*}$ in place of $P(\sigma)$ ) we had the additional restriction $\tilde{r}>\hat{r}+1$ which for 4.10 would require $1-\tilde{r}_{+}>\hat{r}_{-}+1$, i.e. as $1-\hat{r}_{+}=\hat{r}_{-}$, the requirement translates to $\tilde{r}_{+}<\hat{r}_{+}-1$, which is incompatible with the restriction $\tilde{r}_{+}>\hat{r}_{+}+1$ for 4.9 ) applying (to $P(\sigma)$ ) with $\tilde{r}=\tilde{r}_{+}$, i.e. (4.9) does not directly give matching semi-Fredholm estimates.

To fix this, for $\beta>1$, we apply 4.9 to $P(\sigma)^{*}$ with $\tilde{r}=\tilde{r}_{-}=\hat{r}_{-}+1+\epsilon=$ $\left(1-\hat{r}_{+}\right)+1+\epsilon=2+\epsilon-\hat{r}_{+}, 0<\epsilon<\beta-1$, and with $l$ replaced by $-l-2$. This satisfies all the requirements for (4.9), and gives for $v \in H_{\mathrm{b}}^{\tilde{r}_{-}-K,-l-2}$ with 
$P(\sigma)^{*} v \in H_{\mathrm{b}}^{\tilde{r}_{-}-1,-l}$ (see the remarks in the second paragraph after 4.9 ) that

$$
\|v\|_{H_{\mathrm{b}}^{\tilde{r}_{-}-, l-2}} \leq C\left(\left\|P(\sigma)^{*} v\right\|_{H_{\mathrm{b}}^{\tilde{r}_{-}-1,-l}}+\|v\|_{H_{\mathrm{b}}^{\tilde{r}--K,-l-2-\delta}}\right) .
$$

Notice that $\tilde{r}_{-} \geq\left(1-\tilde{r}_{+}\right)+2$, so this is in stronger spaces (in terms of differential order) than the desired (4.10). This estimate gives, via approximating the compact inclusion map by a finite rank map, that there are finitely many linear functionals $\ell_{1}, \ldots, \ell_{M} \in\left(H_{\mathrm{b}}^{\tilde{r}_{-},-l-2}\right)^{*}=H_{\mathrm{b}}^{-\tilde{r}_{-}, l+2}$ (identified via the sesquilinear $L^{2}$-pairing) such that 4.11) can be replaced (under unchanged conditions for $v$ ) by

$$
\|v\|_{H_{\mathrm{b}}^{\tilde{r}_{-},-l-2}} \leq C\left(\left\|P(\sigma)^{*} v\right\|_{H_{\mathrm{b}}^{\tilde{r}_{-}-1,-l}}+\sum_{j=1}^{M}\left|\ell_{j}(v)\right|\right) ;
$$

indeed one may assume (by approximating the finite rank operator) that $\ell_{j} \in$ $\dot{\mathcal{C}}^{\infty}(X)$.

In order to proceed, it is useful to rewrite 4.12 as an estimate without an error term for a slightly different operator. So let, with $R$ in the differential order at this point arbitrary,

$$
\tilde{P}(\sigma): H_{\mathrm{b}}^{R, l} \oplus \mathbb{C}^{M} \rightarrow H_{\mathrm{b}}^{R-2, l+2}
$$

be given by

$$
\tilde{P}(\sigma)(u, c)=P(\sigma) u+\sum c_{j} \ell_{j}
$$

so the formal adjoint is

$$
\tilde{P}(\sigma)^{*} v=\left(P(\sigma)^{*} v, \ell(v)\right),
$$

where $\ell=\left(\ell_{1}, \ldots, \ell_{M}\right)$. Our estimate is, for any $v \in H_{\mathrm{b}}^{\tilde{r}_{-}-K,-l-2}$ with $\tilde{P}(\sigma)^{*} v \in$ $H_{\mathrm{b}}^{\tilde{r}_{-}-1,-l} \oplus \mathbb{C}^{M}$,

$$
\|v\|_{H_{\mathrm{b}}^{\tilde{r}_{-},-l-2}} \leq C\left\|\tilde{P}(\sigma)^{*} v\right\|_{H_{\mathrm{b}}^{\tilde{r}_{-}-1,-l} \oplus \mathbb{C}^{M}} .
$$

In particular, this is valid for all $v \in H_{\mathrm{b}}^{\tilde{r}_{-}+1,-l-2}$, the set of which is dense in $H_{\mathrm{b}}^{\tilde{r}_{-},-l-2}$. Thus, by duality, namely Hahn-Banach in a Hilbert space-setting, defining a continuous linear functional on the range of $\tilde{P}(\sigma)^{*}$ on $H_{\mathrm{b}}^{\tilde{r}_{-}+1,-l-2}$, extended uniquely to the closure of the range in $H_{\mathrm{b}}^{\tilde{r}_{-}-1,-l} \oplus \mathbb{C}^{M}$ (without changing the constant of the estimate), and then the whole space using an orthogonal projection (thus again without changing the constant), one can solve $\tilde{P}(\sigma)(u, c)=f \in$ $H_{\mathrm{b}}^{-\tilde{r}_{-}, l+2}$ with a uniform bound:

$$
\|(u, c)\|_{H_{\mathrm{b}}^{1-\tilde{r}_{-}, l} \oplus \mathbb{C}^{M}} \leq C\|f\|_{H_{\mathrm{b}}^{-\tilde{r}_{-}, l+2}},
$$

with $C$ independent of $\sigma$ in a compact set. But this means that

$$
P(\sigma) u=f-\sum_{j} c_{j} \ell_{j}
$$

and

$$
\|u\|_{H_{\mathrm{b}}^{1-\tilde{r}_{-}, l}} \leq C\|f\|_{H_{\mathrm{b}}^{-\tilde{r}_{-}, l+2}}, \quad\left|c_{j}\right| \leq C\|f\|_{H_{\mathrm{b}}^{-\tilde{r}_{-}, l+2}}
$$

(with $C$ independent of $f$ and of $\sigma$ ).

Now, if $f \in H_{\mathrm{b}}^{\tilde{r}_{+}-1, l+2}$ (note that $H_{\mathrm{b}}^{\tilde{r}_{+}-1, l+2} \subset H_{\mathrm{b}}^{-\tilde{r}_{-}, l+2}$ as $\tilde{r}_{-} \geq\left(1-\tilde{r}_{+}\right)+2 \geq$ $\left.1-\tilde{r}_{+}\right)$then the regularity estimates of Section 3 , see Proposition 3.5 , which required 
the regularization argument, apply, and as $\sum c_{j} \ell_{j} \in \dot{\mathcal{C}}^{\infty}(X)$, show that $u \in H_{\mathrm{b}}^{\tilde{r}_{+}, l}$, and indeed

$$
\begin{aligned}
\|u\|_{H_{\mathrm{b}}^{\tilde{r}_{+}, l}} & \leq C^{\prime}\left(\left\|f-\sum c_{j} \ell_{j}\right\|_{H_{\mathrm{b}}^{\tilde{r}_{+}-1, l+2}}+\|u\|_{H_{\mathrm{b}}^{1-\tilde{r}_{-}, l}}\right) \\
& \leq C^{\prime}\left(\|f\|_{H_{\mathrm{b}}^{\tilde{r}_{+}-1, l+2}}+\sum_{j}\left|c_{j}\right|+\|u\|_{H_{\mathrm{b}}^{1-\tilde{r}_{-}, l}}\right) .
\end{aligned}
$$

Hence, by 4.14

$$
\|u\|_{H_{\mathrm{b}}^{\tilde{r}+, l}} \leq C^{\prime \prime}\left(\|f\|_{H_{\mathrm{b}}^{\tilde{r}_{+}-1, l+2}}+\|f\|_{H_{\mathrm{b}}^{-\tilde{r}_{-}, l+2}}\right) \leq C^{\prime \prime \prime}\|f\|_{H_{\mathrm{b}}^{\tilde{r}_{+}-1, l+2}}
$$

with $C^{\prime \prime \prime}$ independent of $f$ and $\sigma$.

Now, for $v \in H_{\mathrm{b}}^{1-\tilde{r}_{+},-l-2}$ with $P(\sigma)^{*} v \in H_{\mathrm{b}}^{-\tilde{r}_{+},-l}$,

$$
\begin{aligned}
|\langle f, v\rangle| & =\left|\left\langle(u, c), \tilde{P}(\sigma)^{*} v\right\rangle\right| \leq\|(u, c)\|_{H_{\mathrm{b}}^{\tilde{r}_{+}, l}}\left\|\tilde{P}(\sigma)^{*} v\right\|_{H_{\mathrm{b}}^{-\tilde{r}_{+},-l}} \\
& \leq C^{\prime \prime \prime}\|f\|_{H_{\mathrm{b}}^{\tilde{r}_{+}^{-1, l+2}}}\left\|\tilde{P}(\sigma)^{*} v\right\|_{H_{\mathrm{b}}^{-\tilde{r}_{+},-l}}
\end{aligned}
$$

where the equality of the first two expressions is justified by a simple regularization argument in the decay order for $v$. Since $f$ is arbitrary in $H_{\mathrm{b}}^{\tilde{r}_{+}-1, l+2}$, this gives (for $v \in H_{\mathrm{b}}^{1-\tilde{r}_{+},-l-2}$ with $\left.P(\sigma)^{*} v \in H_{\mathrm{b}}^{-\tilde{r}_{+},-l}\right)$

$$
\begin{aligned}
\|v\|_{H_{\mathrm{b}}^{1-\tilde{r}_{+},-l-2}} & \leq C^{\prime \prime \prime}\left\|\tilde{P}(\sigma)^{*} v\right\|_{H_{\mathrm{b}}^{-\tilde{r}_{+},-}} \\
& \leq C\left(\left\|P(\sigma)^{*} v\right\|_{H_{\mathrm{b}}^{-\tilde{r}_{+},-}}+\sum_{j}\left|\ell\left(v_{j}\right)\right|\right),
\end{aligned}
$$

which immediately implies the desired estimate (4.10). Thus, we achieved our second goal and proved (4.10) in the case $\tilde{r}_{+}>\hat{r}+1$. In particular, given (4.7), this proves Proposition 4.1 in case $\tilde{r}>\hat{r}+1$.

It remains to extend to range of $\tilde{r}$ to all functions allowed in the statement of Proposition 4.1. The final step of the proof of Proposition 4.1 is to remove the restriction $\tilde{r}>\hat{r}+1$.

We break this final part into two steps. We next prove Proposition 4.1 in case $\tilde{r}<\hat{r}-1$.

As we already discussed, $P(\sigma)$ and $P(\sigma)^{*}$ have analogous properties as pseudodifferential operators, so we can interpret (4.10) as an estimate for $P(\sigma)$, namely

$$
\|u\|_{H_{\mathrm{b}}^{1-\tilde{r}_{+},-l-2}} \leq C\left(\|P(\sigma) u\|_{H_{\mathrm{b}}^{-\tilde{r}_{+},-l}}+\|u\|_{H_{\mathrm{b}}^{1-\tilde{r}_{+}-K,-l-2-\delta}}\right),
$$

which, with $\tilde{r}_{-}=1-\tilde{r}_{+}$is the estimate

$$
\|u\|_{H_{\mathrm{b}}^{\tilde{r}_{-},-l-2}} \leq C\left(\|P(\sigma) u\|_{H_{\mathrm{b}}^{\tilde{r}_{-}-1,-l}}+\|u\|_{\left.H_{\mathrm{b}}^{\tilde{r}_{-}-K,-l-2-\delta}\right)}\right)
$$

now $\tilde{r}_{-}<-\hat{r}_{+}=\hat{r}_{-}-1$, i.e. (recall that the role of the \pm sign choices is completely symmetric) the estimate 4.2

$$
\|u\|_{H_{\mathrm{b}}^{\tilde{r}, l}} \leq C\left(\|P(\sigma) u\|_{H_{\mathrm{b}}^{\tilde{r}-1, l+2}}+\|u\|_{\left.H_{\mathrm{b}}^{\tilde{r}-K, l-\delta}\right)} ;\right.
$$

in the low-regularity case $\tilde{r}<\hat{r}-1$. Since in this case we already have the dual semi-Fredholm estimate, this proves Proposition 4.1 if $\tilde{r}<\hat{r}-1$.

We now remove all restrictions on $\tilde{r}$ beyond those of the statement of Proposition 4.1. With $\tilde{r}=\tilde{r}_{+}$arbitrary in Proposition 4.1. we are going to go through the first part of the argument again, starting with (4.1):

$$
\|u\|_{H_{\mathrm{b}}^{\tilde{r}_{+}, l}} \leq C\left(\|P(\sigma) u\|_{H_{\mathrm{b}}^{\tilde{r}_{+}-1, l+2}}+\|u\|_{H_{\mathrm{b}}^{-N, l}}\right),
$$


with $N>\beta+1, \beta>1$ and $-N<\tilde{r}_{+}$. But now we estimate the error term $\|u\|_{H_{\mathrm{b}}^{-N, l}}$ using 4.16 with $-N \leq \tilde{r}<\min \left(\hat{r}_{+}(\beta)-1, \tilde{r}_{+}\right)$to obtain

$$
\|u\|_{H_{\mathrm{b}}^{\tilde{r}}+, l} \leq C\left(\|P(\sigma) u\|_{H_{\mathrm{b}}^{\tilde{r}_{+}-1, l+2}}+\|P(\sigma) u\|_{H_{\mathrm{b}}^{\tilde{\mathrm{r}}-1, l+2}}+\|u\|_{H_{\mathrm{b}}^{\tilde{r}-K, l-\delta}}\right),
$$

and thus the general case of the estimate 4.2 of Proposition 4.1. Since this also applies to $P(\sigma)^{*}$ on the dual spaces, this completes the proof of Proposition 4.1.

4.2. Reduction of the normal operator estimate, Proposition 4.6, to a positivity computation. Thus, it suffices to prove Proposition 4.6, which we recall is the estimate

$$
\|v\|_{H_{\mathrm{b}}^{\hat{r}, l}} \leq C\|\tilde{N}(P(\sigma)) v\|_{H_{\mathrm{b}}^{\hat{r}-1, l+2}}
$$

where

$$
\hat{r}=\frac{1}{2}-(l+1) \pm \beta \frac{\tau_{\mathrm{b}}}{\left(\tau_{\mathrm{b}}^{2}+\left|\mu_{\mathrm{b}}\right|^{2}\right)^{1 / 2}}, \quad \beta>0 .
$$

For this purpose it is more convenient to work with $L_{\mathrm{b}}^{2}$, so we set $\tilde{H}_{\mathrm{b}}$ to be the b-Sobolev space relative to $L_{\mathrm{b}}^{2}$, here this really is of interest in $[0, \infty) \times \partial X$, with density $\frac{d x}{x} d g_{\partial X}$. Our argument will proceed by showing that a modification of the commutator (3.3) in $\Psi_{\mathrm{b}}^{2 \hat{r}, 2 l}$ that we considered in the proof of (4.1) actually can be arranged to have a positive normal operator as well, relative to $L^{2}$, which statement is equivalent to saying that $x^{-n}$ times this normal operator is positive relative to $L_{\mathrm{b}}^{2}$ since the quadratic form on $L_{\mathrm{b}}^{2}$ is $\left\langle x^{n} \cdot, \cdot\right\rangle_{g}$. This will immediately imply (4.17). Now, if we write

$$
N(A)=x^{n / 2} \tilde{A} x^{-n / 2} x^{-l-1},
$$

$\tilde{A} \in \Psi_{\mathrm{b}}^{\hat{r}-1 / 2,0}$ on the model space $[0, \infty) \times \partial X$, dilation invariant, then we need to compute

$$
\begin{aligned}
& i\left(\tilde{N}\left(P(\sigma)^{*}-P(\sigma)\right)\right) N\left(A^{*} A\right)+i\left[\tilde{N}(P(\sigma)), N\left(A^{*} A\right)\right] \\
& \quad=-2 \operatorname{Im}\left(\sigma^{2}\right) x^{-n / 2-l-1} \tilde{A}^{*} x^{n} \tilde{A} x^{-n / 2-l-1}+i\left[\Delta_{\mathrm{sc}}, x^{-n / 2-l-1} \tilde{A}^{*} x^{n} \tilde{A} x^{-n / 2-l-1}\right],
\end{aligned}
$$

to the extent that we can show its positivity (or negativity) on $L^{2}$ (more precisely a lower bound for \pm this operator by $C x^{-2 l}, C>0$ ). As mentioned above, this is equivalent to

$$
-2 \operatorname{Im}\left(\sigma^{2}\right) x^{-n} x^{-n / 2-l-1} \tilde{A}^{*} x^{n} \tilde{A} x^{-n / 2-l-1}+i x^{-n}\left[\Delta_{\mathrm{sc}}, x^{-n / 2-l-1} \tilde{A}^{*} x^{n} \tilde{A} x^{-n / 2-l-1}\right]
$$

having the corresponding sign on $L_{\mathrm{b}}^{2}$, with the explicit lower bound now being $C x^{-2(l+n / 2)}$. The reason for the extra factor $x^{-n / 2}$ conjugating $\tilde{A}$ in our definition of $\tilde{A}$ is that if $\tilde{A}$ is symmetric relative to the $L_{\mathrm{b}}^{2}$ inner product, as we arrange to simplify our arguments, then $x^{n / 2} \tilde{A} x^{-n / 2}$ is symmetric with respect to the $L^{2}$-inner product, so in fact we have to compute

$$
\begin{aligned}
& -2 \operatorname{Im}\left(\sigma^{2}\right) x^{-n} x^{-l-1}\left(x^{n / 2} \tilde{A} x^{-n / 2}\right)^{2} x^{-l-1}+i x^{-n}\left[\Delta_{\mathrm{sc}}, x^{-l-1}\left(x^{n / 2} \tilde{A} x^{-n / 2}\right)^{2} x^{-l-1}\right] \\
& =-2 \operatorname{Im}\left(\sigma^{2}\right) x^{-n / 2-l-1} \tilde{A}^{2} x^{-n / 2-l-1}+i x^{-n}\left[\Delta_{\mathrm{sc}}, x^{n / 2-l-1} \tilde{A}^{2} x^{-n / 2-l-1}\right] .
\end{aligned}
$$

Notice that the first term here is a negative operator (in an indefinite sense) if $\operatorname{Im}\left(\sigma^{2}\right) \geq 0$, and a positive operator if $\operatorname{Im}\left(\sigma^{2}\right) \leq 0$, so if in the first case we arrange that the second term is negative definite (in an appropriate sense), while in the second case we arrange that the second term is positive definite, in an estimate 
one may simply drop the first term, i.e. allowing complex $\sigma$ with $\sigma^{2}$ of the correct imaginary part does not affect the argument below.

Let

$$
\Delta_{\mathrm{b}}=x^{-(n+2) / 2} \Delta_{\mathrm{sc}} x^{(n-2) / 2} \in \operatorname{Diff}_{\mathrm{b}}^{2}
$$

as $x^{-1} \Delta_{\mathrm{sc}} x^{-1}$ is symmetric with respect to the $L^{2}$-inner product, $\Delta_{\mathrm{b}}$ is symmetric with respect to the $L_{\mathrm{b}}^{2}$ inner product. Explicitly,

$$
\begin{aligned}
\Delta_{\mathrm{b}} & =x^{n / 2} D_{x} x^{-n+3} D_{x} x^{n / 2-1}+\Delta_{\partial X} \\
& =\left(D_{x} x+i \frac{n}{2}\right) x^{-n / 2+2} D_{x} x^{n / 2-1}+\Delta_{\partial X} \\
& =\left(D_{x} x+i \frac{n}{2}\right)\left(x D_{x}-i \frac{n-2}{2}\right)+\Delta_{\partial X} \\
& =\left(x D_{x}\right)^{2}+\Delta_{\partial X}+\left(\frac{n-2}{2}\right)^{2} ;
\end{aligned}
$$

notice that this is a positive definite operator on $L_{\mathrm{b}}^{2}$ for $n \geq 3$, since on the Mellin transform side it is multiplication by a positive function.

Now

$$
\begin{aligned}
& i x^{-n}\left[\Delta_{\mathrm{sc}}, x^{n / 2-l-1} \tilde{A}^{2} x^{-n / 2-l-1}\right] \\
& =i x^{-n} x^{\frac{n+2}{2}} \Delta_{\mathrm{b}} x^{-\frac{n-2}{2}} x^{n / 2-l-1} \tilde{A}^{2} x^{-n / 2-l-1} \\
& \quad-i x^{-n} x^{n / 2-l-1} \tilde{A}^{2} x^{-n / 2-l-1} x^{\frac{n+2}{2}} \Delta_{\mathrm{b}} x^{-\frac{n-2}{2}},
\end{aligned}
$$

whose positivity is equivalent to that of the operator obtained by multiplying from both sides by $x^{l+n / 2}$ (chosen to make the total weight $x^{0}$, this also changes the desired lower bound to a positive constant):

$$
i x^{l+1} \Delta_{\mathrm{b}} x^{-l} \tilde{A}^{2} x^{-1}-i x^{-1} \tilde{A}^{2} x^{-l} \Delta_{\mathrm{b}} x^{l+1} \in \Psi_{\mathrm{b}}^{2 \hat{r}, 0} .
$$

But $\Delta_{\mathrm{b}}$ and $\tilde{A}$ are both dilation invariant pseudodifferential operators, so the effect of conjugating them by $x^{k}$ (i.e. multiplying by this from the right, and by its inverse from the left) is replacing $x D_{x}$ by $x D_{x}-i k$, or on the Mellin transform side replacing $\tau_{\mathrm{b}}$ by $\tau_{\mathrm{b}}-i k$. Writing such a change by affixing $(\cdot-i k)$ to the operator, we need to compute

$$
\begin{aligned}
i x^{l+1} & \Delta_{\mathrm{b}} x^{-l} \tilde{A}^{2} x^{-1}-i x^{-1} \tilde{A}^{2} x^{-l} \Delta_{\mathrm{b}} x^{l+1} \\
& =i \Delta_{\mathrm{b}}(\cdot+i(l+1)) \tilde{A}(\cdot+i)^{2}-i \tilde{A}(\cdot-i)^{2} \Delta_{\mathrm{b}}(\cdot-i(l+1)) .
\end{aligned}
$$

Now all operators on the right hand side are multiplication operators on the Mellin transform side (no $x$ dependence), so if we choose $\tilde{A}$ to depend on $y$ and its $b$ dual variables only through $\Delta_{\partial X}$, and still symmetric with respect to the $L_{\mathrm{b}}^{2}$-inner product, then the positivity or negativity of this expression is a commutative calculation, and the expression can be written as (with imaginary part on the second line meaning skew-adjoint part, which becomes the imaginary part on the Mellin transform/spectral side)

$$
\begin{aligned}
& i \tilde{A}(\cdot+i)^{2} \Delta_{\mathrm{b}}(\cdot+i(l+1))-i \tilde{A}(\cdot-i)^{2} \Delta_{\mathrm{b}}(\cdot-i(l+1)) \\
& =-2 \operatorname{Im} \tilde{A}(\cdot+i)^{2} \Delta_{\mathrm{b}}(\cdot+i(l+1)) .
\end{aligned}
$$

Notice that if 4.22 is positive (with the negative case essentially the same), i.e. on the Mellin transform side, replacing $\Delta_{\partial X}$ by its spectral parameter $\lambda \geq 0$, is given by multiplication by a positive function, which is the square of a positive elliptic symbol $\tilde{b}$ of order $\hat{r}$ in terms of $\left(\tau_{\mathrm{b}}, \sqrt{\lambda}\right.$ ) (with the square root present due 
to $\Delta_{\partial X}$ being second order), then one has (for $\operatorname{Im}\left(\sigma^{2}\right) \leq 0$; or $\operatorname{Im}\left(\sigma^{2}\right) \geq 0$ in the negative case)

$$
x^{-n} x^{l+n / 2}\left(\left(\tilde{N}\left(P(\sigma)^{*}-P(\sigma)\right)\right) N\left(A^{*} A\right)+\left[\tilde{N}(P(\sigma)), N\left(A^{*} A\right)\right]\right) x^{l+n / 2} \geq \tilde{B}^{{ }^{\mathrm{b}}} \tilde{B},
$$

with $\tilde{B} \in \Psi_{\mathrm{b}}^{\hat{r}, 0}$, with $\tilde{B}^{* \mathrm{~b}}$ the adjoint in $L_{\mathrm{b}}^{2}$ (and is $=\tilde{B}$ ), namely $\tilde{B}$ has Mellin transformed normal operator given by the functional calculus of $\Delta_{\partial X}$ for $\tilde{b}$. Here the inequality is due to both merely using a lower bound for (4.22) and to dropping the $\operatorname{Im}\left(\sigma^{2}\right)$ term, which has a sign matching that of 4.22$)$. Thus, with the inner product now the $L^{2}$-based, so $\tilde{B}^{*}=x^{n} \tilde{B}^{*_{\mathrm{b}}} x^{-n}=x^{n} \tilde{B} x^{-n}$, and with $B=x^{n / 2} \tilde{B} x^{-l-n / 2}$, so $B^{*}=x^{-l-n / 2} \tilde{B}^{*} x^{n / 2}=x^{n} x^{-l-n / 2} \tilde{B}^{*_{\mathrm{b}}} x^{n / 2} x^{-n}$, we have

$$
\begin{aligned}
\|B u\|^{2} & \leq\langle i N(A) u, N(A) \tilde{N}(P(\sigma)) u\rangle-\langle i N(A) \tilde{N}(P(\sigma)) u, N(A) u\rangle \\
& \leq C\|N(A) u\|_{H_{\mathrm{b}}^{1 / 2,-1}}\|N(A) \tilde{N}(P(\sigma)) u\|_{H_{\mathrm{b}}^{-1 / 2,1}} \\
& \leq C\|u\|_{H_{\mathrm{b}}^{\hat{r},}, l}\|\tilde{N}(P(\sigma)) u\|_{H_{\mathrm{b}}^{\hat{\mathrm{r}}-1, l+2}} .
\end{aligned}
$$

But by the positivity of $\tilde{B},\|B u\|$ is equivalent to $\|u\|_{H_{\mathrm{b}}^{\hat{r}, l}}$, so dividing 4.23 by the latter proves the weaker estimate (4.7) instead of the desired 4.2 .

In summary, we have proved (4.17), thus Proposition 4.6, and hence Proposition 4.1. if we show that we can choose $A$ so that the positivity of (4.22) holds, with a lower bound by the square of a positive elliptic symbol of order $\hat{r}$ on the Mellin transform/spectral side.

4.3. Choice of the operator $\tilde{A}$ and completion of the proof of Proposition 4.6. In order to obtain a positive 4.22, we arrange below that the principal symbol of $A$ is, for suitable $\tilde{\beta}>0$ to be chosen, and with $\psi$ identically 1 near 0 , of sufficiently small support as in (3.4),

$$
\begin{aligned}
a=x^{-l-1} \psi(x) \exp \left( \pm \frac{\tilde{\beta}}{2}\right. & \frac{\tau_{\mathrm{b}}}{\left(\tau_{\mathrm{b}}^{2}+\left|\mu_{\mathrm{b}}\right|^{2}\right)^{1 / 2}} \\
& +\left( \pm \frac{\beta}{2} \frac{\tau_{\mathrm{b}}}{\left(\tau_{\mathrm{b}}^{2}+\left|\mu_{\mathrm{b}}\right|^{2}\right)^{1 / 2}}\right) \log \left(\tau_{\mathrm{b}}^{2}+\left|\mu_{\mathrm{b}}\right|^{2}\right) \\
& \left.-\frac{l+1}{2} \log \left(\tau_{\mathrm{b}}^{2}+\left|\mu_{\mathrm{b}}\right|^{2}\right)\right),
\end{aligned}
$$

which is

$$
\exp \left( \pm \frac{\tilde{\beta}}{2} \frac{\tau_{\mathrm{b}}}{\left(\tau_{\mathrm{b}}^{2}+\left|\mu_{\mathrm{b}}\right|^{2}\right)^{1 / 2}}\right)
$$

times the principal symbol used for our symbolic computation in (3.4) (which uses the same $\tilde{r}=\hat{r}$ as here), and is the same as that employed in the more general computation (3.16). Much as in the more general $\tilde{r}$ setting of Proposition 3.5, this extra factor will give us sufficient flexibility in order to ensure the normal operator positivity. Notice that it automatically ensures the symbolic positivity of the normal operator, i.e. that it is positive modulo compact terms, and moreover it can be bounded below by the square of a pseudodifferential operator modulo compact terms, in accordance with the proof of Proposition 3.5. cf. the discussion around (3.10) for dropping logarithmic terms. 
With this motivation, in accordance with 4.24, we may almost take $\tilde{A}$ to be the Mellin conjugate of

$$
\begin{aligned}
\exp \left( \pm \frac{\tilde{\beta}}{2}\right. & \frac{\tau_{\mathrm{b}}}{\left(\tau_{\mathrm{b}}^{2}+\Delta_{\partial X}+\tilde{\digamma}^{2}\right)^{1 / 2}} \\
& +\left( \pm \frac{\beta}{2} \frac{\tau_{\mathrm{b}}}{\left(\tau_{\mathrm{b}}^{2}+\Delta_{\partial X}+\digamma^{2}\right)^{1 / 2}}\right) \log \left(\tau_{\mathrm{b}}^{2}+\Delta_{\partial X}+\digamma^{2}\right) \\
& \left.-\frac{l+1}{2} \log \left(\tau_{\mathrm{b}}^{2}+\Delta_{\partial X}+\check{\digamma}^{2}\right)\right)
\end{aligned}
$$

where

$$
\tilde{\digamma} \geq \digamma \geq \check{\digamma}>1, \tilde{\beta} \geq 0
$$

are parameters, and where the square root and the logarithm are defined with branch cuts along the negative real axis, and are real for positive arguments. Here 'almost' refers to that with this choice we do not have an entire (operator-valued) function on the Mellin transform side, rather simply holomorphic in a strip, of width $2 \check{\digamma}$ around the real axis; this is easily remedied, see Lemma 4.9 . Indeed, in the spectral representation of $\Delta_{\partial X} \geq 0$ it may be replaced by a non-negative spectral parameter $\lambda$, and then, for complex $\tau_{\mathrm{b}}$,

$$
\operatorname{Re}\left(\tau_{\mathrm{b}}^{2}+\lambda+\check{\digamma}^{2}\right) \geq \check{\digamma}^{2}-\left(\operatorname{Im} \tau_{\mathrm{b}}\right)^{2},
$$

so is positive in $\left|\operatorname{Im} \tau_{\mathrm{b}}\right|<\check{\digamma}$, and similarly for $\check{\digamma}$ replaced by $\digamma \tilde{\digamma} \tilde{\digamma}$, and thus 4.25 indeed gives a holomorphic function in this strip. Note that 4.22 makes sense for all $\check{\digamma}>1$ in view of the domain of holomorphy. Notice also that 4.25 does have the correct principal symbol, i.e. behavior as $\left(\tau_{\mathrm{b}}, \mu_{\mathrm{b}}\right) \rightarrow \infty$, namely that given by (4.24).

While this formula looks complicated, we mention already now that in the important case of $l+1=0$, which we discuss separately below, it simplifies significantly: one can take $\tilde{\beta}=0$, so only the second term in the exponent is non-trivial. Otherwise, in general, we take $\tilde{\beta}$ to be actually positive, indeed

$$
\tilde{\beta}=\frac{\pi}{2} \tilde{\digamma}
$$

when $l+1 \neq 0$. We also remark that while the $\tilde{\beta}$ term (being a symbol of order 0 , thus bounded) does not affect the order of $\tilde{A}$, it does affect the principal symbol when $\tilde{\beta}>0$; cf. Corollary 4.11 .

We start by remarking that 4.25 ) is defined via the functional calculus, which gives a family of pseudodifferential operators due to the Cauchy-Stokes formula of Helffer-Sjöstrand [21] that expresses the function as an integral involving the resolvent and an almost analytic extension of the function, see 20 for a treatment. Indeed, it is the quantization of a symbol of class $S_{1-\delta^{\prime}, \delta^{\prime}}^{\tilde{r}-1 / 2}$ for all $\delta^{\prime} \in(0,1)$, with principal symbol

$$
\begin{aligned}
\exp \left( \pm \frac{\tilde{\beta}}{2}\right. & \frac{\tau_{\mathrm{b}}}{\left(\tau_{\mathrm{b}}^{2}+\left|\mu_{\mathrm{b}}\right|^{2}+\tilde{\digamma}^{2}\right)^{1 / 2}} \\
& +\left( \pm \frac{\beta}{2} \frac{\tau_{\mathrm{b}}}{\left(\tau_{\mathrm{b}}^{2}+\left|\mu_{\mathrm{b}}\right|^{2}+\digamma^{2}\right)^{1 / 2}}\right) \log \left(\tau_{\mathrm{b}}^{2}+\left|\mu_{\mathrm{b}}\right|^{2}+\digamma^{2}\right) \\
& \left.-\frac{l+1}{2} \log \left(\tau_{\mathrm{b}}^{2}+\left|\mu_{\mathrm{b}}\right|^{2}+\check{\digamma}^{2}\right)\right)
\end{aligned}
$$


which is symbolic jointly in $\left(\tau_{\mathrm{b}}, \mu_{\mathrm{b}}\right)$ in $\left|\operatorname{Im} \tau_{\mathrm{b}}\right|<\check{\digamma}$. In fact, if we regard $\check{\digamma}, \digamma, \tilde{\digamma}$ as large parameters, notice that the three terms arising by taking the logarithm have joint symbolic properties. Namely

$$
\frac{\tau_{\mathrm{b}}}{\left(\tau_{\mathrm{b}}^{2}+\left|\mu_{\mathrm{b}}\right|^{2}+\tilde{\digamma}^{2}\right)^{1 / 2}}
$$

is symbolic jointly in $\left(\tau_{\mathrm{b}}, \mu_{\mathrm{b}}, \tilde{F}\right)$ (with $y$ as the 'base variable' which is in a compact set, entering via the dual metric) of order zero, and similarly

$$
\frac{\tau_{\mathrm{b}}}{\left(\tau_{\mathrm{b}}^{2}+\left|\mu_{\mathrm{b}}\right|^{2}+\digamma^{2}\right)^{1 / 2}} \log \left(\tau_{\mathrm{b}}^{2}+\left|\mu_{\mathrm{b}}\right|^{2}+\digamma^{2}\right)
$$

is symbolic jointly in $\left(\tau_{\mathrm{b}}, \mu_{\mathrm{b}}, \digamma\right)$ of any positive (indeed logarithmic) order, and

$$
\log \left(\tau_{\mathrm{b}}^{2}+\left|\mu_{\mathrm{b}}\right|^{2}+\check{\digamma}^{2}\right)
$$

is symbolic jointly in $\left(\tau_{\mathrm{b}}, \mu_{\mathrm{b}}, \check{\digamma}\right)$ of any positive order. In addition:

Lemma 4.7. In $\left|\operatorname{Im} \tau_{\mathrm{b}}\right|<\check{\digamma}$, the imaginary part of (4.27), resp. 4.28), resp. (4.29), is, for all $\delta^{\prime}>0$, a symbol of order $-1+\delta^{\prime}$ in $\left(\tau_{\mathrm{b}}, \mu_{\mathrm{b}}, \tilde{\digamma}\right)$, resp. $\left(\tau_{\mathrm{b}}, \mu_{\mathrm{b}}, \digamma\right)$, resp. $\left(\tau_{\mathrm{b}}, \mu_{\mathrm{b}}, \check{\digamma}\right)$ (in fact, in the first and last cases order -1$)$.

Furthermore, on the line $\operatorname{Im} \tau_{\mathrm{b}}=1$, the principal symbol of the imaginary part of 4.27), resp. 4.28), resp. 4.29), is

$$
\left(\tau_{\mathrm{b}}^{2}+\left|\mu_{\mathrm{b}}\right|^{2}+\tilde{\digamma}^{2}\right)^{-3 / 2}\left(\left|\mu_{\mathrm{b}}\right|^{2}+\tilde{\digamma}^{2}\right) \text {, modulo } S^{-3} \text {. }
$$

resp.

$$
\left(\tau_{\mathrm{b}}^{2}+\left|\mu_{\mathrm{b}}\right|^{2}+\digamma^{2}\right)^{-3 / 2}\left(\left(\left|\mu_{\mathrm{b}}\right|^{2}+\digamma^{2}\right) \log \left(\tau_{\mathrm{b}}^{2}+\left|\mu_{\mathrm{b}}\right|^{2}+\digamma^{2}\right)+2 \tau_{\mathrm{b}}^{2}\right) \text {, modulo } S^{-3+\delta^{\prime}},
$$

resp.

$$
2 \tau_{\mathrm{b}}\left(\tau_{\mathrm{b}}^{2}+\left|\mu_{\mathrm{b}}\right|^{2}+\check{\digamma}^{2}\right)^{-1}, \text { modulo } S^{-3} .
$$

Thus, the principal symbols at $\operatorname{Im} \tau_{\mathrm{b}}=1$, when regarded as a symbol in $\left(\tau_{\mathrm{b}}, \mu_{\mathrm{b}}\right)$ (i.e. $\check{\digamma}, \digamma, \tilde{\digamma}$ are fixed or in a compact set), are

$$
\left(\tau_{\mathrm{b}}^{2}+\left|\mu_{\mathrm{b}}\right|^{2}\right)^{-3 / 2}\left|\mu_{\mathrm{b}}\right|^{2}
$$

resp.

$$
\left(\tau_{\mathrm{b}}^{2}+\left|\mu_{\mathrm{b}}\right|^{2}\right)^{-3 / 2}\left(\left|\mu_{\mathrm{b}}\right|^{2} \log \left(\tau_{\mathrm{b}}^{2}+\left|\mu_{\mathrm{b}}\right|^{2}\right)+2 \tau_{\mathrm{b}}^{2}\right),
$$

resp.

$$
2 \tau_{\mathrm{b}}\left(\tau_{\mathrm{b}}^{2}+\left|\mu_{\mathrm{b}}\right|^{2}\right)^{-1}
$$

modulo $S^{-3}$, resp. $S^{-3+\delta^{\prime}}$, resp. $S^{-3}$.

Remark 4.8. The relevance of $\operatorname{Im} \tau_{\mathrm{b}}=1$ is due to the $+i$ in $(4.22)$.

In addition, as the principal symbols already indicate, in fact the symbol is only logarithmically bigger than one of order -1 .

Proof. Since the imaginary part vanishes for real $\tau_{\mathrm{b}}$ for all these holomorphic functions, we express it as the integral, from the real axis along the imaginary direction, of their derivative; this is an integral over an interval of bounded length $(=1)$. But the derivative is a symbol of order $-1+\delta^{\prime}$ for all $\delta^{\prime}>0$, so the conclusion of being a symbol of this order follows immediately. The actual principal symbol arises by simply integrating the principal symbol of the derivative. To see the more precise 
conclusion regarding the error, namely that it is (almost) two orders lower than the principal term, note that for $g$ real on the reals, writing $\partial_{\operatorname{Im} \tau_{\mathrm{b}}}=i \partial_{\tau_{\mathrm{b}}}$ for the derivative along the imaginary direction, by Taylor's formula,

$$
\begin{gathered}
2 i \operatorname{Im} g\left(\tau_{\mathrm{b}}+i\right)=g\left(\tau_{\mathrm{b}}+i\right)-g\left(\tau_{\mathrm{b}}-i\right)=\left(g\left(\tau_{\mathrm{b}}+i\right)-g\left(\tau_{\mathrm{b}}\right)\right)-\left(g\left(\tau_{\mathrm{b}}-i\right)-g\left(\tau_{\mathrm{b}}\right)\right) \\
=\left(\partial_{\operatorname{Im} \tau_{\mathrm{b}}} g\left(\tau_{\mathrm{b}}\right)+\frac{1}{2} \partial_{\operatorname{Im} \tau_{\mathrm{b}}}^{2} g\left(\tau_{\mathrm{b}}\right)+\frac{1}{2} \int_{0}^{1}(1-s)^{2} \partial_{\operatorname{Im} \tau_{\mathrm{b}}}^{3} g\left(\tau_{\mathrm{b}}+i s\right) d s\right) \\
\quad-\left(-\partial_{\operatorname{Im} \tau_{\mathrm{b}}} g\left(\tau_{\mathrm{b}}\right)+\frac{1}{2} \partial_{\operatorname{Im} \tau_{\mathrm{b}}}^{2} g\left(\tau_{\mathrm{b}}\right)-\frac{1}{2} \int_{0}^{1}(1-s)^{2} \partial_{\operatorname{Im} \tau_{\mathrm{b}}}^{3} g\left(\tau_{\mathrm{b}}-i s\right) d s\right) \\
=2\left(\partial_{\operatorname{Im} \tau_{\mathrm{b}}} g\left(\tau_{\mathrm{b}}\right)+\frac{1}{2} \int_{0}^{1}(1-s)^{2}\left(\partial_{\operatorname{Im} \tau_{\mathrm{b}}}^{3} g\left(\tau_{\mathrm{b}}+i s\right)+\partial_{\operatorname{Im} \tau_{\mathrm{b}}}^{3} g\left(\tau_{\mathrm{b}}-i s\right)\right) d s\right.
\end{gathered}
$$

and the third derivatives listed are in $S^{-3+\delta^{\prime}}$ in all cases and in $S^{-3}$ in the first and last cases.

The aforementioned limited domain of holomorphy actually suffices for the argument by choosing sufficiently large $\check{\digamma}>1$ and working with a large b-calculus, with still sufficient decay at the left and right boundaries (given by $\partial X$ ) so that the departure from the small calculus is irrelevant. However, it is in any case straightforward to fix this absence of being entire: simply convolve 4.25 with a Gaussian, $\frac{1}{\sqrt{\pi s}} e^{-\tau_{\mathrm{b}}^{2} /(2 s)}$, which does not change the principal symbol:

Lemma 4.9. Suppose that with $\tilde{A}$ replaced by the Mellin conjugate of multiplication by 4.25, (4.22) is positive, resp. negative, with a lower bound given by the square of a symbol on the Mellin transform/spectral side. Then, for sufficiently small $s>0$, letting $\tilde{A}$ be the Mellin conjugate of multiplication by the convolution of 4.25 with $\frac{1}{\sqrt{\pi s}} e^{-\tau_{\mathrm{b}}^{2} /(2 s)}, \tilde{A} \in \Psi_{\mathrm{b}}^{\tilde{r}-1 / 2,0}$ and the same positivity, resp. negativity, along with the symbolic property, holds.

Thus, in order to prove Proposition 4.6, it suffices to show the positivity or negativity of 4.22 for the choice of multiplication by 4.25 as the candidate for the Mellin conjugate of $\tilde{A}$; the actual choice will arise by a convolution with a Gaussian.

Proof. On the Schwartz kernel side, with boundary defining functions $x, x^{\prime}$ in the left and right factors, the convolution by $\frac{1}{\sqrt{\pi s}} e^{-\tau_{\mathrm{b}}^{2} /(2 s)}$ corresponds to multiplication by a function which, being a Gaussian in terms of $\log \left(x / x^{\prime}\right)$, is superexponentially decaying, and thus in terms of the defining functions of the two 'side faces', locally $x / x^{\prime}$ and $x^{\prime} / x$ in the regions where these are bounded, is superpolynomially decaying. (See Section 2 for a discussion of the decay of the residual terms; note that the symbolic term, 2.1), has compact support in $t-t^{\prime}$, thus $x / x^{\prime}$ and $x^{\prime} / x$ are bounded above on it. Note also that such multiplication leaves the principal symbol unaffected as the multiplication is by a function that is 1 at the diagonal.) While this convolution changes 4.25, the asymptotic behavior (thus positivity) of the Mellin conjugate of 4.22 , as $\left|\left(\tau_{\mathrm{b}}, \lambda\right)\right| \rightarrow \infty$, is unaffected (for any value of $s>0$, and indeed in a uniform sense as $s \rightarrow 0$ ), moreover letting $s \rightarrow 0$ the convolution converges to 4.25 uniformly on compact sets, so in view of the asymptotic positivity, for sufficiently small $s>0$ the convolution results in a function that is 
both entire (with appropriate estimates) and positive, so that $\tilde{A} \in \Psi_{\mathrm{b}}^{\tilde{r}-1 / 2,0}$ indeed and the desired positivity holds.

With the choice 4.25 on the Mellin transform side, we work on the spectral side of $\Delta_{\partial X}$, and we may replace the latter by its spectral parameter $\lambda \geq 0$. Then $\tilde{A}$ becomes a multiplication operator by

$$
\begin{aligned}
f\left(\tau_{\mathrm{b}}\right)=f_{\lambda}\left(\tau_{\mathrm{b}}\right)=\exp \left( \pm \frac{\tilde{\beta}}{2}\right. & \frac{\tau_{\mathrm{b}}}{\left(\tau_{\mathrm{b}}^{2}+\lambda+\tilde{\digamma}^{2}\right)^{1 / 2}} \\
& +\left( \pm \frac{\beta}{2} \frac{\tau_{\mathrm{b}}}{\left(\tau_{\mathrm{b}}^{2}+\lambda+\digamma^{2}\right)^{1 / 2}}\right) \log \left(\tau_{\mathrm{b}}^{2}+\lambda+\digamma^{2}\right) \\
& \left.-\frac{l+1}{2} \log \left(\tau_{\mathrm{b}}^{2}+\lambda+\check{\digamma}^{2}\right)\right)
\end{aligned}
$$

where we consider $\lambda$ a non-negative parameter and suppress it in the notation, together with the other parameters $\beta, \tilde{\beta}, \digamma, \tilde{\digamma}, \check{\digamma}$. In order to show the positivity, resp. negativity, of 4.22, by 4.22 and 4.21, it suffices to show that

$$
-2 \operatorname{Im}\left(f\left(\tau_{\mathrm{b}}+i\right)^{2}\left(\left(\tau_{\mathrm{b}}+i(l+1)\right)^{2}+\lambda+\left(\frac{n-2}{2}\right)^{2}\right)\right)
$$

is positive, resp. negative, (everywhere) for appropriate choices of the constants $\tilde{\beta}$, $\check{\digamma}, \digamma$ and $\tilde{\digamma}$. This can be achieved, via taking logarithms, by showing that

$$
\begin{gathered}
\operatorname{Im}\left( \pm \tilde{\beta} \frac{\tau_{\mathrm{b}}+i}{\left(\left(\tau_{\mathrm{b}}+i\right)^{2}+\lambda+\tilde{\digamma}^{2}\right)^{1 / 2}}\right) \\
+\operatorname{Im}\left(\left( \pm \beta \frac{\tau_{\mathrm{b}}+i}{\left(\left(\tau_{\mathrm{b}}+i\right)^{2}+\lambda+\digamma^{2}\right)^{1 / 2}}\right) \log \left(\left(\tau_{\mathrm{b}}+i\right)^{2}+\lambda+\digamma^{2}\right)\right) \\
+\operatorname{Im}\left(-(l+1) \log \left(\left(\tau_{\mathrm{b}}+i\right)^{2}+\lambda+\check{\digamma}^{2}\right)\right) \\
+\operatorname{Im} \log \left(\left(\tau_{\mathrm{b}}+i(l+1)\right)^{2}+\lambda+\left(\frac{n-2}{2}\right)^{2}\right)
\end{gathered}
$$

is in $(0, \pi)$ (for the negative sign conclusion in 4.38 ), corresponding to $\beta>0$ ), resp. in $(-\pi, 0)$ (for the positive sign conclusion in 4.38), corresponding to $\beta<0$ ).

For the last term, observe here that:

Lemma 4.10. Suppose $|l+1|<\frac{n-2}{2}$. Let $\lambda=\nu^{2}$. Then the last term of 4.39],

$$
\operatorname{Im} \log \left(\left(\tau_{\mathrm{b}}+i(l+1)\right)^{2}+\nu^{2}+\left(\frac{n-2}{2}\right)^{2}\right),
$$

is a symbol in $\left(\tau_{\mathrm{b}}, \nu\right)$ of order -1 , with principal symbol

$$
\frac{2(l+1) \tau_{\mathrm{b}}}{\tau_{\mathrm{b}}^{2}+\nu^{2}}, \text { modulo } S^{-3} \text {. }
$$

In addition, there is $\alpha_{0} \in(0, \pi / 2)$ such that 4.40 lies in $\left[-\alpha_{0}, \alpha_{0}\right]$.

Furthermore, if $l+1=0$, 4.40 vanishes.

Proof. Since

$$
\left.\operatorname{Re}\left(\left(\tau_{\mathrm{b}}+i(l+1)\right)^{2}+\lambda+\left(\frac{n-2}{2}\right)^{2}\right)\right)=\tau_{\mathrm{b}}^{2}-(l+1)^{2}+\left(\frac{n-2}{2}\right)^{2}>0,
$$

4.40 , which is the argument of the complex number whose real part is displayed, is in $\left(-\frac{\pi}{2}, \frac{\pi}{2}\right)$. 
In addition, much like in Lemma 4.7, as $\log \left(\tau_{\mathrm{b}}^{2}+\nu^{2}+\left(\frac{n-2}{2}\right)^{2}\right)$ is real on the real axis, is holomorphic in $\left|\operatorname{Im} \tau_{\mathrm{b}}\right|<\frac{n-2}{2}$, and its derivative is a symbol of order -1 in $\left(\tau_{\mathrm{b}}, \nu\right)$, its imaginary part is also a symbol of order -1 in this strip, thus tends to 0 as $\left|\left(\tau_{\mathrm{b}}, \nu\right)\right| \rightarrow \infty$. Since the derivative is

$$
2 \tau_{\mathrm{b}}\left(\tau_{\mathrm{b}}^{2}+\nu^{2}+\left(\frac{n-2}{2}\right)^{2}\right)^{-1}
$$

integrating from the real axis to the line with imaginary part $l+1$ gives the principal symbol claim (in this sense the constant term is irrelevant). The improved error term (the modulo $S^{-3}$ statement) follows from 4.36 in the proof of Lemma 4.7 .

Thus, 4.40 is bounded away from the endpoints of the interval $\left(-\frac{\pi}{2}, \frac{\pi}{2}\right)$ since as $\left|\left(\tau_{\mathrm{b}}, \nu\right)\right| \rightarrow \infty$ it tends to 0 , and in a compact region this boundedness claim automatically holds.

The last part follows since in this case 4.40 is the argument of a positive number.

As a consequence of Lemma 4.7 and Lemma 4.10 we have:

Corollary 4.11. For any $\tilde{\digamma} \geq \digamma \geq \check{\digamma}>1$, the expression 4.39 is, for all $\delta^{\prime}>0$, a symbol of order $-1+\delta^{\prime}$ in $\left(\tau_{\mathrm{b}}, \nu\right)$, with principal symbol

$$
\pm \beta\left(\tau_{\mathrm{b}}^{2}+\nu^{2}\right)^{-3 / 2}\left(\nu^{2} \log \left(\tau_{\mathrm{b}}^{2}+\nu^{2}\right)+2 \tau_{\mathrm{b}}^{2}\right) \pm \tilde{\beta}\left(\tau_{\mathrm{b}}^{2}+\nu^{2}\right)^{-3 / 2} \nu^{2},
$$

and is thus positive/negative depending on the \pm sign. Thus, for any such $\digamma, \tilde{\digamma}, \check{\digamma}$, 4.39) lies in $(0, \pi)$, resp. $(-\pi, 0)$, indeed in an arbitrarily small specified one-sided neighborhood of 0 , for sufficiently large $\left(\tau_{\mathrm{b}}, \nu\right)$.

Remark 4.12. We emphasize that we are computing the argument (imaginary part of the logarithm), not the imaginary part of the function multiplication by which gives the modified (by multiplication by powers of $x$ ) normal operator 4.22 on the Mellin transform/spectral side. The actual imaginary part of that function is a symbol of order $2 \tilde{r}+\delta^{\prime}$ for all $\delta^{\prime}>0$, corresponding to the real part of the function being a symbol of order $2(\tilde{r}-1 / 2)+2=2 \tilde{r}+1$.

Proof. We just need to observe that, by using the above expressions for the principal symbols of the four summands, the principal symbol of 4.39 is

$$
\begin{aligned}
& \pm \tilde{\beta}\left(\tau_{\mathrm{b}}^{2}+\nu^{2}\right)^{-3 / 2} \nu^{2} \\
& \quad \pm \beta\left(\tau_{\mathrm{b}}^{2}+\nu^{2}\right)^{-3 / 2}\left(\nu^{2} \log \left(\tau_{\mathrm{b}}^{2}+\nu^{2}\right)+2 \tau_{\mathrm{b}}^{2}\right) \\
& \quad-(l+1) 2 \tau_{\mathrm{b}}\left(\tau_{\mathrm{b}}^{2}+\nu^{2}\right)^{-1} \\
& \quad+\frac{2(l+1) \tau_{\mathrm{b}}}{\tau_{\mathrm{b}}^{2}+\nu^{2}} \\
& = \pm \beta\left(\tau_{\mathrm{b}}^{2}+\nu^{2}\right)^{-3 / 2}\left(\nu^{2} \log \left(\tau_{\mathrm{b}}^{2}+\nu^{2}\right)+2 \tau_{\mathrm{b}}^{2}\right) \pm \tilde{\beta}\left(\tau_{\mathrm{b}}^{2}+\nu^{2}\right)^{-3 / 2} \nu^{2}
\end{aligned}
$$

which is positive, up to the \pm sign. The final part follows as symbols of negative order tend to 0 at infinity.

Note that when $|l+1|$ is close to $\frac{n-2}{2}$, 4.40 can be arbitrarily close to both of $\pi / 2,-\pi / 2$, and does so for $\tau_{\mathrm{b}}$ very close to 0 , which makes the treatment of this problem harder since the other terms in 4.39 need to be in a very precisely controlled interval around $\pi / 2$ so that the sum is in $(0, \pi)$. 
Notice that $l+1=0$ corresponds to the 'symmetric' (in terms of weight) mapping $H_{\mathrm{b}}^{\tilde{r},-1} \rightarrow H_{\mathrm{b}}^{\tilde{r}-1,1}$. The last statement in Lemma 4.10 makes the $l+1=0$ case a bit simpler, so we discuss it first. (If one takes $\beta>0$ small, it can easily be made completely explicit, without the use of large parameters, though this deducts from the simplicity, and moreover we need to allow $\beta>1$ in the statement of Proposition 4.6 in order to prove Proposition 4.1.)

4.3.1. The case $l+1=0$ and preliminary computations for the general case. If $l+1=0$, we simply take $\tilde{\beta}=0$, so (in addition to the third and fourth terms) the first term of (4.39) also vanishes (and $\tilde{\digamma}$ becomes irrelevant), and we need to estimate the second term only. This will be useful for the general case as well, so we state it as a lemma.

Lemma 4.13. The second term of 4.39, with the notation $\lambda=\nu^{2}$, and without the $\beta-\tilde{\beta}$ prefactor,

$$
I I=\operatorname{Im}\left(\left(\frac{\tau_{\mathrm{b}}+i}{\left(\left(\tau_{\mathrm{b}}+i\right)^{2}+\nu^{2}+\digamma^{2}\right)^{1 / 2}}\right) \log \left(\left(\tau_{\mathrm{b}}+i\right)^{2}+\nu^{2}+\digamma^{2}\right)\right),
$$

satisfies the following: let $\varepsilon>0$. There exists $\digamma_{*}>0$ such that for $\digamma \geq \digamma_{*}$, $I I \in(0, \varepsilon)$ for all $\left(\tau_{\mathrm{b}}, \nu\right)$.

Corollary 4.14. For any $\beta \neq 0$ there exists $\digamma_{*}>0$ such that for $\digamma \geq \digamma_{*}$, 4.39, is in $(0, \pi)$ if $\beta>0$, and 4.39 is in $(-\pi, 0)$ if $\beta<0$.

Proof. Take $\varepsilon=\pi /(2|\beta|)$, and apply Lemma 4.13 , noting that, with $\tilde{\beta}=0$, the only non-zero term of 4.39 is the second one, which is in $(0, \pi)$.

Proof of Lemma 4.13. In the large parameter sense, the principal symbol of 4.41) is

$$
\left(\tau_{\mathrm{b}}^{2}+\nu^{2}+\digamma^{2}\right)^{-3 / 2}\left(\left(\nu^{2}+\digamma^{2}\right) \log \left(\tau_{\mathrm{b}}^{2}+\nu^{2}+\digamma^{2}\right)+2 \tau^{2}\right)
$$

modulo $S^{-3+\delta^{\prime}}, \delta^{\prime}>0$, thus for any $\delta^{\prime}>0$, 4.41) differs from this principal symbol, in absolute value, by

$$
\leq C_{1}\left(\tau_{\mathrm{b}}^{2}+\nu^{2}+\digamma^{2}\right)^{-3 / 2+\delta^{\prime}} \leq C_{1} \digamma^{-1+2 \delta^{\prime}}\left(\tau_{\mathrm{b}}^{2}+\nu^{2}+\digamma^{2}\right)^{-1}
$$

with $C_{1}$ independent of $\digamma$. On the other hand, for $\digamma \geq 2,4.42$ has a lower bound

$$
\left(\tau_{\mathrm{b}}^{2}+\nu^{2}+\digamma^{2}\right)^{-3 / 2}\left(\left(\nu^{2}+\digamma^{2}\right) \log \left(\tau_{\mathrm{b}}^{2}+\nu^{2}+\digamma^{2}\right)+2 \tau^{2}\right) \geq\left(\tau_{\mathrm{b}}^{2}+\nu^{2}+\digamma^{2}\right)^{-1 / 2}
$$

since the $\operatorname{logarithm}$ is $\geq 2 \log \digamma \geq 1$, and an upper bound

$$
2\left(\tau_{\mathrm{b}}^{2}+\nu^{2}+\digamma^{2}\right)^{-1 / 2} \log \left(\tau_{\mathrm{b}}^{2}+\nu^{2}+\digamma^{2}\right) \leq C_{2}\left(\tau_{\mathrm{b}}^{2}+\nu^{2}+\digamma^{2}\right)^{-1 / 4} .
$$

Thus, in view of 4.43, there is $\digamma_{*}>2$ such that for $\digamma>\digamma_{*}$, 4.41 has a lower bound

$$
\frac{1}{2}\left(\tau_{\mathrm{b}}^{2}+\nu^{2}+\digamma^{2}\right)^{-1 / 2}>0
$$

and an upper bound

$$
3\left(\tau_{\mathrm{b}}^{2}+\nu^{2}+\digamma^{2}\right)^{-1 / 2} \log \left(\tau_{\mathrm{b}}^{2}+\nu^{2}+\digamma^{2}\right) \leq C_{2} \digamma^{-1 / 2}<\varepsilon .
$$

This proves the lemma. 
This corollary implies that for in case $l+1=0$, with $\beta>0$ arbitrary (and $\tilde{\beta}=0$ ), with $\digamma$ chosen sufficiently large (depending on $\beta$ ) $(4.38)$, thus 4.22 , are indeed negative. In particular, Proposition 4.6 holds in this case, completing the proof of Theorem 1.1 when $l+1=0$.

Also note that the positivity result for $l+1=0$ (choosing $\digamma$ depending on $\beta$, and then allowing $l+1$ of size depending on these two choices) proves an analogous result for $|l+1|$ small since 4.38 will have an unchanged sign.

4.3.2. The case of general $l+1$. In the general case of $|l+1|<\frac{n-2}{2}$ we first choose $\check{\digamma}>1$ so that the sum of the last two terms is still in a compact subinterval of $(-\pi / 2, \pi / 2)$, see Lemma 4.15, then choose $\digamma$ sufficiently large so that the same property remains when the second term is added, and in addition for sufficiently large $\tau_{\mathrm{b}}^{2}+\nu^{2}$ this sum is positive, and indeed close to 0 , see Corollary 4.17, and then let $\tilde{\beta}=\frac{\pi}{2} \tilde{\digamma}$ and choose $\tilde{\digamma}$ sufficiently large so that the first term is close to the constant $\pi / 2$ on the compact region of $\left(\tau_{\mathrm{b}}, \nu\right)$ where we had not established positivity and is bounded below by a suitably small negative quantity everywhere, bounded above by a constant slightly greater than $\pi / 2$, so that the total sum is in $(0, \pi)$, see Lemma 4.18 and Corollary 4.19

We thus prove:

Lemma 4.15. There exists $\check{\digamma}_{*}>1$ such that for $\check{\digamma} \geq \check{\digamma}_{*}$, the last two terms of 4.39) (with the notation $\lambda=\nu^{2}$ ),

$$
\begin{aligned}
\operatorname{Im}( & \left.-(l+1) \log \left(\left(\tau_{\mathrm{b}}+i\right)^{2}+\nu^{2}+\check{\digamma}^{2}\right)\right) \\
& +\operatorname{Im} \log \left(\left(\tau_{\mathrm{b}}+i(l+1)\right)^{2}+\nu^{2}+\left(\frac{n-2}{2}\right)^{2}\right),
\end{aligned}
$$

have opposite signs (in the sense that if one is $\geq 0$, the other is $\leq 0$ ), the sum lies in a compact subinterval $\left[-\alpha_{0}, \alpha_{0}\right]$ of $(-\pi / 2, \pi / 2)$, and is a symbol of order -3 in $\left(\tau_{\mathrm{b}}, \nu\right)$.

Thus, there exist $C_{0} \geq 1$ such that for $\left|\left(\tau_{\mathrm{b}}, \nu\right)\right| \geq 1$, the absolute value of the sum is $\leq C_{0}\left|\left(\tau_{\mathrm{b}}, \nu\right)\right|^{-3}$.

Remark 4.16. The upper bound in the last statement is only useful on $\left|\left(\tau_{\mathrm{b}}, \nu\right)\right| \geq R_{0}$ where $C_{0} R_{0}^{-3}=\alpha_{0}$, i.e. $R_{0}=C_{0}^{1 / 3} / \alpha_{0}^{1 / 3}$.

Proof. Since $\operatorname{Im}\left(\left(\tau_{\mathrm{b}}+i(l+1)\right)^{2}+\nu^{2}+\left(\frac{n-2}{2}\right)^{2}\right)=2(l+1) \tau_{\mathrm{b}}$,

$$
\operatorname{Im} \log \left(\left(\tau_{\mathrm{b}}+i(l+1)\right)^{2}+\nu^{2}+\left(\frac{n-2}{2}\right)^{2}\right) \in(-\pi / 2, \pi / 2)
$$

is $\geq 0$, resp. $\leq 0$ when $2(l+1) \tau_{\mathrm{b}} \geq 0$, resp. $2(l+1) \tau_{\mathrm{b}} \leq 0$.

On the other hand, $\operatorname{Im}\left(\left(\tau_{\mathrm{b}}+i\right)^{2}+\nu^{2}+\check{\digamma}^{2}\right)=2 \tau_{\mathrm{b}}$, hence has the sign of $\tau_{\mathrm{b}}$, and thus, as $\operatorname{Re}\left(\left(\tau_{\mathrm{b}}+i\right)^{2}+\nu^{2}+\check{\digamma}^{2}\right)=\tau_{\mathrm{b}}^{2}-1+\nu^{2}+\check{\digamma}^{2}>0, \operatorname{Im} \log \left(\left(\tau_{\mathrm{b}}+\right.\right.$ $\left.i)^{2}+\nu^{2}+\check{\digamma}^{2}\right)$ is in $[0, \pi / 2)$, resp. $(-\pi / 2,0]$ corresponding to whether $\tau_{\mathrm{b}} \geq 0$ or $\tau_{\mathrm{b}} \leq 0$. Moreover, by choosing $\check{\digamma}>1$ sufficiently large, one can arrange that $\left|\operatorname{Im} \log \left(\left(\tau_{\mathrm{b}}+i\right)^{2}+\nu^{2}+\check{\digamma}^{2}\right)\right|<\frac{\pi}{2(1+|l+1|)}$, as follows from the large parameter symbolic considerations from Lemma 4.7 namely that the left hand side is bounded by $C\left(\tau_{\mathrm{b}}^{2}+\nu^{2}+\check{\digamma}^{2}\right)^{-1 / 2} \leq C / \check{\digamma}$, with $C$ independent of $\check{\digamma}$. (It is also straightforward 
to arrange this explicitly.) Thus,

$\operatorname{Im}\left(-(l+1) \log \left(\left(\tau_{\mathrm{b}}+i\right)^{2}+\nu^{2}+\check{\digamma}^{2}\right)\right) \in\left(-\frac{\pi|l+1|}{2(1+|l+1|)}, \frac{\pi|l+1|}{2(1+|l+1|)}\right) \subset\left(-\frac{\pi}{2}, \frac{\pi}{2}\right)$,

with sign matching that of $-(l+1) \tau_{\mathrm{b}}$, thus indeed the opposite of that of the second term of 4.45.

Since the sum of two quantities of opposite sign, each of which is in $(-\pi / 2, \pi / 2)$, is itself in $(-\pi / 2, \pi / 2)$, and since the asymptotic behavior of each term is that of a symbol of order -1 , thus the terms decay to 0 , the conclusion regarding $\alpha_{0}$ follows. The principal symbol as a symbol of order -1 is the sum of two principal symbols, which are $\pm 2(l+1) \frac{\tau_{\mathrm{b}}}{\tau_{\mathrm{b}}^{2}+\nu^{2}}$, the sum has vanishing principal symbol modulo $S^{-3}$, and thus it is a symbol of order -3 . The final statement is an immediate consequence of this symbolic property.

Then:

Corollary 4.17. Let $\check{\digamma}_{*}>1$ be as in Lemma 4.15. There exist $\digamma_{*}>0$ and $\alpha_{*} \in(0, \pi / 2)$ such that for $\digamma \geq \digamma_{*}$ the sum of the last three terms of $(4.39)$ is in $\left[-\alpha_{*}, \alpha_{*}\right]$ and there is $R>0$ such that for $\tau_{\mathrm{b}}^{2}+\nu^{2} \geq R^{2}$, the sum of these last three terms is $>\frac{1}{8}\left(\tau_{\mathrm{b}}^{2}+\nu^{2}+\digamma^{2}\right)^{-1 / 2}>0$.

Proof. This is immediate using Lemma 4.15 first, then choosing $\varepsilon=\left(\pi / 2-\alpha_{0}\right) /(4|\beta|)$ in Lemma 4.13 to find $\digamma_{*}$. Since the second term of 4.39 is positive and less than $|\beta| \varepsilon=\left(\pi / 2-\alpha_{0}\right) / 4$, the sum of the last three terms lies in $\left[-\alpha_{*}, \alpha_{*}\right]$ with $0<\alpha_{*}=\alpha_{0}+\left(\pi / 2-\alpha_{0}\right) / 4<\pi / 2$. Finally, due to 4.44, the second term has a lower bound $\frac{1}{2}\left(\tau_{\mathrm{b}}^{2}+\nu^{2}+\digamma^{2}\right)^{-1 / 2}$, which is bounded from below by $\frac{1}{4}\left(\tau_{\mathrm{b}}^{2}+\nu^{2}\right)^{-1 / 2}$ for $\left|\left(\tau_{\mathrm{b}}, \nu\right)\right| \geq \digamma$, while the sum of the last two terms has an upper bound in absolute value $\leq C_{0}\left|\left(\tau_{\mathrm{b}}, \nu\right)\right|^{-3}$ (where $\left|\left(\tau_{\mathrm{b}}, \nu\right)\right|>1$ ), so for sufficiently large $\left|\left(\tau_{\mathrm{b}}, \nu\right)\right|$ (namely, $\left.\left|\left(\tau_{\mathrm{b}}, \nu\right)\right| \geq R=\max \left(\digamma, \sqrt{8 C_{0}}\right)\right)$, the second term dominates, and thus the last two terms can be absorbed into it as desired, giving the statement of the corollary.

Hence, at this point, we have that the sum of the last three terms is contained in $(0, \pi)$ for $\tau_{\mathrm{b}}^{2}+\nu^{2} \geq R^{2}$, and is contained in a compact subinterval of $(-\pi / 2, \pi / 2)$ for all $\left(\tau_{\mathrm{b}}, \nu\right)$.

Lemma 4.18. There exists $\tilde{\digamma}_{*} \geq \digamma_{*}$ such that for $\tilde{\digamma} \geq \tilde{\digamma}_{*}$, the first term of [4.39], with the $\tilde{\beta}=\frac{\pi}{2} \tilde{\digamma}$ prefactor,

$$
\operatorname{Im}\left(\tilde{\beta} \frac{\tau_{\mathrm{b}}+i}{\left(\left(\tau_{\mathrm{b}}+i\right)^{2}+\nu^{2}+\tilde{\digamma}^{2}\right)^{1 / 2}}\right),
$$

is $\geq-\frac{1}{16}\left(\tau_{\mathrm{b}}^{2}+\nu^{2}+\digamma^{2}\right)^{-1 / 2}$, is $<\frac{\pi}{2}+\frac{1}{4}\left(\frac{\pi}{2}-\alpha_{*}\right)$ everywhere, and is $>\alpha_{*}$ for $\tau_{\mathrm{b}}^{2}+\nu^{2} \leq R^{2}$.

Proof. In the large parameter sense, the principal symbol of 4.46 is, without the $\tilde{\beta}$ prefactor,

$$
\left(\tau_{\mathrm{b}}^{2}+\nu^{2}+\tilde{\digamma}^{2}\right)^{-3 / 2}\left(\nu^{2}+\tilde{\digamma}^{2}\right)
$$

modulo $S^{-3}$, which is non-negative; including the prefactor it becomes, as a symbol of order 0 now jointly in $\left(\tau_{\mathrm{b}}, \nu, \tilde{\digamma}\right)$,

$$
\frac{\pi}{2} \tilde{\digamma}\left(\tau_{\mathrm{b}}^{2}+\nu^{2}+\tilde{\digamma}^{2}\right)^{-3 / 2}\left(\nu^{2}+\tilde{\digamma}^{2}\right)
$$


modulo $S^{-2}$. But, using $\tilde{\digamma} \geq \digamma$,

$$
\begin{gathered}
\frac{\pi}{2} \tilde{\digamma}\left|\operatorname{Im}\left(\frac{\tau_{\mathrm{b}}+i}{\left(\left(\tau_{\mathrm{b}}+i\right)^{2}+\nu^{2}+\tilde{\digamma}^{2}\right)^{1 / 2}}\right)-\left(\tau_{\mathrm{b}}^{2}+\nu^{2}+\tilde{\digamma}^{2}\right)^{-3 / 2}\left(\nu^{2}+\tilde{\digamma}^{2}\right)\right| \\
\leq C \tilde{\boldsymbol{\digamma}}\left(\tau_{\mathrm{b}}^{2}+\nu^{2}+\tilde{\digamma}^{2}\right)^{-3 / 2} \leq C \tilde{\digamma}^{-1}\left(\tau_{\mathrm{b}}^{2}+\nu^{2}+\digamma^{2}\right)^{-1 / 2}
\end{gathered}
$$

which is $\leq \frac{1}{16}\left(\tau_{\mathrm{b}}^{2}+\nu^{2}+\digamma^{2}\right)^{-1 / 2}$ for sufficiently large $\tilde{\digamma}$ (say, $\tilde{\digamma} \geq \tilde{\digamma}_{*}^{\prime}$ ). In view of (4.48) being positive, this proves that (4.46) is $\geq-\frac{1}{16}\left(\tau_{\mathrm{b}}^{2}+\nu^{2}+\digamma^{2}\right)^{-1 / 2}$.

The upper bound $\frac{\pi}{2}+\frac{1}{4}\left(\frac{\pi}{2}-\alpha_{*}\right)$ is proved similarly, for $(4.48)$ is bounded from above by $\frac{\pi}{2}$ and $(4.49)$ is bounded from above by $C \tilde{\digamma}^{-1}$, so for sufficiently large $\tilde{\digamma}$, say $\tilde{\boldsymbol{\digamma}} \geq \tilde{\digamma}_{*}^{\prime \prime}$, is $<\frac{1}{4}\left(\frac{\pi}{2}-\alpha_{*}\right)$.

Finally, 4.46 is

$$
\frac{1}{2} \int_{-1}^{1} \frac{\pi}{2} \tilde{\digamma}\left(\left(\tau_{\mathrm{b}}+i s\right)^{2}+\nu^{2}+\tilde{\digamma}^{2}\right)^{-3 / 2}\left(\nu^{2}+\tilde{\digamma}^{2}\right) d s
$$

so with $\hat{\tau}_{\mathrm{b}}=\tau_{\mathrm{b}} / \tilde{\digamma}, \hat{\nu}=\nu / \tilde{\digamma}$,

$$
=\frac{\pi}{4} \int_{-1}^{1}\left(\left(\hat{\tau}_{\mathrm{b}}+i s \tilde{\digamma}^{-1}\right)^{2}+\hat{\nu}^{2}+1\right)^{-3 / 2}\left(\hat{\nu}^{2}+1\right) d s
$$

which is an equicontinuous family in $\tilde{\digamma}^{-1}<2^{-1 / 2}$ (say), taking the value $\frac{\pi}{2}$ at $\hat{\tau}_{\mathrm{b}}=\hat{\nu}=0, \tilde{\digamma}^{-1}=0$. Hence, for any $\varepsilon^{\prime}>0$, such as $\varepsilon^{\prime}=\left(\pi / 2-\alpha_{*}\right) / 4$, there is $\hat{R}>0$, which one may assume to be $<R / 2$, such that for $\hat{\tau}_{\mathrm{b}}^{2}+\hat{\nu}^{2} \leq \hat{R}^{2}$ (and any $\tilde{\digamma})$ it differs from $\frac{\pi}{2}$ by $<\varepsilon^{\prime}$, thus for $\left|\left(\tau_{\mathrm{b}}, \nu\right)\right| \leq \hat{R} \tilde{\digamma}$ the analogous conclusion holds.

Now simply pick $\tilde{\digamma}_{*}^{\prime \prime \prime}=R / \hat{R}>2$, and let $\tilde{\digamma}_{*}=\max \left(\tilde{\digamma}_{*}^{\prime}, \tilde{\digamma}_{*}^{\prime \prime}, \tilde{\digamma}_{*}^{\prime \prime \prime}\right)$. Then the $\geq-\frac{1}{16}\left(\tau_{\mathrm{b}}^{2}+\nu^{2}+\digamma^{2}\right)^{-1 / 2},<\frac{\pi}{2}+\frac{1}{4}\left(\frac{\pi}{2}-\alpha_{*}\right)$, and $>\alpha_{*}$ for $\tau_{\mathrm{b}}^{2}+\nu^{2} \leq R^{2}$, claims follow immediately.

Corollary 4.19. With $\digamma \geq \digamma_{*}, \tilde{\digamma} \geq \tilde{\digamma}_{*}, \tilde{\beta}=\frac{\pi}{2} \tilde{\digamma}$, 4.39 lies in $(0, \pi)$.

Proof. Since by Corollary 4.17 the sum of the last three terms lies in $\left(-\pi / 2, \alpha_{*}\right]$, $\alpha_{*}<\pi / 2$, while the first is $\leq \pi / 2+\left(\pi / 2-\alpha_{*}\right) / 4$, certainly the sum is $<\pi$ everywhere. Moreover, for $\tau_{\mathrm{b}}^{2}+\nu^{2} \leq R^{2}$, the sum of the first three terms is $\geq-\alpha_{*}$, while the first term is $>\alpha_{*}$, so the sum is positive. Finally, for $\tau_{\mathrm{b}}^{2}+\nu^{2} \geq R^{2}$, the sum of the last three terms is $\geq \frac{1}{8}\left(\tau_{\mathrm{b}}^{2}+\nu^{2}+\digamma^{2}\right)$, while the first is $\geq-\frac{1}{16}\left(\tau_{\mathrm{b}}^{2}+\nu^{2}+\digamma^{2}\right)$, so the sum is $\geq \frac{1}{16}\left(\tau_{\mathrm{b}}^{2}+\nu^{2}+\digamma^{2}\right)$, thus positive, as desired.

This corollary implies that for $\beta>0$ arbitrary, with $\tilde{\digamma}, \digamma, \check{\digamma}$ chosen sufficiently large (depending on $\beta$ ) (4.38), thus (4.22), are indeed negative. In particular, this completes the proof of Proposition 4.6, and thus that of Theorem 1.1. as well.

\section{SECOND Microlocal ANALYSis}

We now consider a second microlocal scattering version of Theorem 4.5. The reason for this is that for $\sigma \neq 0$, it is simplest to consider the limiting resolvent as a map between variable order scattering spaces. One can extend such a picture to $\sigma=0$ if one resolves the zero section by blowing it up; this gives rise to the second microlocal statement below.

First, recall the basic sc-analysis ingredients from [27, keeping in mind that these arise by generalizing the structure of $\mathbb{R}^{n}$ near infinity by identifying asymptotically conic open sets first with open sets in the radial compactification $\overline{\mathbb{R}^{n}}$ of $\mathbb{R}^{n}$, and then the latter with open sets on the compact manifold with boundary $X$. This 
is completely analogous to how pseudodifferential operators are transplanted from $\mathbb{R}^{n}$ to manifolds via coordinate charts; the off-diagonal smooth contributions to the Schwartz kernel in that case are replaced by Schwartz contributions in the present case (i.e. right-densities on $X^{2}$ which vanish to infinite order at $\partial\left(X^{2}\right)$ ); see [45, Section 5.3] for a complete treatment along these lines.

Thus, recall from the introduction that the set (and Lie algebra) of sc-vector fields, $\mathcal{V}_{\mathrm{sc}}(X)$, is $x \mathcal{V}_{\mathrm{b}}(X)$, and is spanned by $x^{2} \partial_{x}, x \partial_{y_{j}}$ over $\mathcal{C}^{\infty}(X)$, i.e. a sc-vector field is a linear combination of $x^{2} \partial_{x}, x \partial_{y_{j}}$ with $\mathcal{C}^{\infty}(X)$ coefficients. Such vector fields are all smooth sections of a vector bundle, ${ }^{\mathrm{sc}} T X$, over $X$; a local basis for the latter is $x^{2} \partial_{x}, x \partial_{y_{j}}$. If $X$ is the radial compactification of $\mathbb{R}^{n}$, i.e. $\mathbb{R}^{n}$ is compactified by gluing $r=+\infty$ to it via identifying $\mathbb{R}^{n} \backslash \overline{B_{1}(0)}$ with $(1, \infty)_{r} \times \mathbb{S}^{n-1}$ via spherical coordinates, introducing $x=r^{-1}$ and adding $x=0$ to the space, so $(1, \infty)_{r} \times \mathbb{S}^{n-1}$ is identified with $(0,1)_{x} \times \mathbb{S}^{n-1}$ in $[0,1)_{x} \times \mathbb{S}^{n-1}$, then $\mathcal{V}_{\mathrm{sc}}(X)$ consists exactly of linear combinations of the coordinate vector fields $\partial_{z_{j}}$ with classical symbolic of order 0 coefficients. Correspondingly, ${ }^{\mathrm{sc}} T \overline{\mathbb{R}^{n}}=\overline{\mathbb{R}_{z}^{n}} \times \mathbb{R}_{v}^{n}$, where over the interior of $\mathbb{R}_{z}^{n}$ one simply writes tangent vectors as $\sum_{j} v_{j} \partial_{z_{j}}$, and the identification actually only uses the vector space structure of $\mathbb{R}^{n}$ (but not the basis, or having a metric).

Dually, the sc-cotangent bundle, ${ }^{\mathrm{sc}} T^{*} X$, is spanned by $\frac{d x}{x^{2}}, \frac{d y_{j}}{x}$ in local coordinates. In case of $X^{\circ}=\mathbb{R}^{n}$, one obtains

$$
{ }^{\mathrm{sc}} T^{*} \overline{\mathbb{R}^{n}}=\overline{\mathbb{R}_{z}^{n}} \times\left(\mathbb{R}^{n}\right)_{\zeta}^{*}
$$

i.e. sc-one-forms are of the form $\sum_{j} a_{j} d z_{j}, a_{j} \in \mathcal{C}^{\infty}(X)=S_{\mathrm{cl}}^{0}\left(\mathbb{R}^{n}\right)$. One writes $\left(\tau, \mu_{j}\right)$ as local coordinates. Below it is sometimes convenient to compactify the fibers of ${ }^{\mathrm{sc}} T^{*} X$ radially, which is possible by exactly the same construction as the compactification of $\mathbb{R}^{n}$; see Figure 3. Thus,

$$
\overline{{ }^{\mathrm{sc}} T^{*} \mathbb{R}^{n}}=\overline{\mathbb{R}_{z}^{n}} \times \overline{\left(\mathbb{R}^{n}\right)_{\zeta}^{*}}
$$

One defines $\operatorname{Diff}_{\mathrm{sc}}(X)$ as finite sums of products of elements of $\mathcal{V}_{\mathrm{sc}}(X)$ with $\mathcal{C}^{\infty}(X)$ coefficients; in case of $\mathbb{R}^{n}$ these are just of the form $\sum_{\alpha} a_{\alpha} D^{\alpha}, a_{\alpha} \in$ $\mathcal{C}^{\infty}(X)=S_{\mathrm{cl}}^{0}\left(\mathbb{R}^{n}\right)$. We also consider general symbolic coefficients, and write $S^{l} \operatorname{Diff}_{\mathrm{sc}}(X)$ for sc-differential operators with symbolic coefficients of order $l$; in the case of $\mathbb{R}^{n}$ again this simply means that $a_{\alpha} \in S^{l}\left(\mathbb{R}^{n}\right)$, the standard space of symbols of order $l$.

Scattering pseudodifferential operators are microlocalizations of these sc-differential operators. They arise as quantizations of symbols on ${ }^{\mathrm{sc}} T^{*} X$; classical symbols of order $(0,0)$ are smooth functions on the fiber-compactification $\overline{{ }^{\overline{ } c} T^{*}} X$. In case of $X^{\circ}=\mathbb{R}^{n}$, the latter are order $(0,0)$ classical symbols in the standard sense, i.e. they have asymptotic expansions at both boundary hypersurfaces. Conversely, their principal symbols are in $S^{m, l}\left({ }^{\mathrm{sc}} T^{*} X\right) / S^{m-1, l-1}\left({ }^{\mathrm{sc}} T^{*} X\right)$, which for classical symbols can be identified with functions on the boundary.

One has Sobolev spaces, including variable order Sobolev spaces, corresponding to this structure; e.g. for $s$ a non-negative integer $H_{\mathrm{sc}}^{s, r}$ consists of distributions such that for all $Q \in \operatorname{Diff}_{\mathrm{sc}}^{s}(X), Q u \in x^{r} L^{2}=x^{r} L_{\mathrm{sc}}^{2}$. Again, via the identification via asymptotically conic coordinate charts in $\mathbb{R}^{n}$, this corresponds to the standard (albeit possibly variable order) Sobolev spaces. We refer to [45, Section 5.3.9] for a detailed discussion.

We also recall, see 27] and [45, Section 5.3], that elliptic theory applies in this sc-setting, and gives for instance the Fredholmness, and via formal self-adjointness 

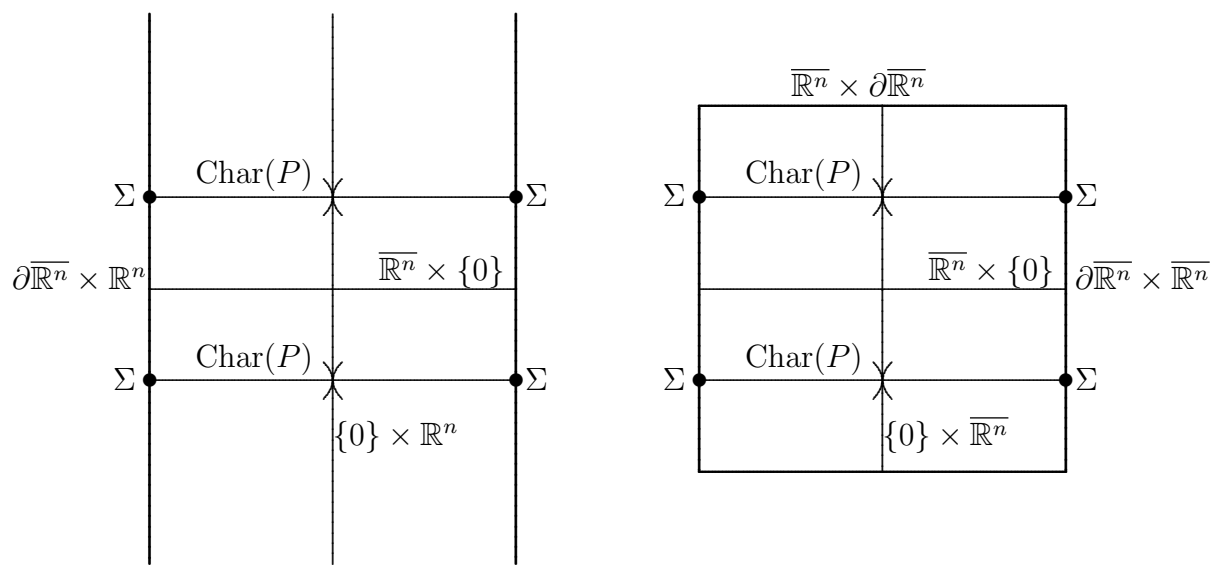

Figure 3 . The scattering cotangent bundle ${ }^{\mathrm{sc}} T^{*} X$ (left) and its fiber-compactification $\overline{{ }^{\mathrm{sc}} T^{*}} X$, with $X=\overline{\mathbb{R}^{n}}$. Also shown is the characteristic set of $P(\sigma)$ for $\sigma \neq 0$, both from the compactified perspective, as $\Sigma$, which is a subset of the boundary, and from the conic perspective, here conic in the base (i.e. the dilations are in the $\mathbb{R}_{z}^{n}$ factor), as Char $(P)$. The fiber of cotangent bundle over the origin, i.e. $\{0\} \times \overline{\left(\mathbb{R}^{n}\right)^{*}}$, is also indicated; this is only special from the conic (dilation) perspective, in which it is the analogue of the zero section in standard microlocal analysis.

invertibility, of $P(\sigma), \sigma \in \mathbb{C} \backslash \mathbb{R}$, as a map

$$
P(\sigma): H_{\mathrm{sc}}^{s+2, r} \rightarrow H_{\mathrm{sc}}^{s, r},
$$

where $s, r$ can be taken to be arbitrary variable orders, and the inverse is independent of the choices, e.g. in the sense that on the dense Schwartz subspace (functions vanishing to infinite order at $\partial X$ ) all inverse agree.

Then the limiting absorption principle, in the Fredholm setting, for $\sigma \neq 0$, and for suitable variable order $r$, namely one monotone along the Hamilton flow and satisfying the appropriate inequalities at the radial sets, with choice irrelevant in the elliptic region (by elliptic theory), and which can be taken to be

$$
r=-\frac{1}{2} \pm \beta \frac{\tau}{\left(\tau^{2}+|\mu|^{2}\right)^{1 / 2}}
$$

near the characteristic set (which is disjoint from the zero section, where (5.1) is singular), and which in case of $\mathbb{R}^{n}$ becomes

$$
r=-\frac{1}{2} \mp \beta \frac{z \cdot \zeta}{|z||\zeta|},
$$

is that

$$
P(\sigma):\left\{u \in H_{\mathrm{sc}}^{s+2, r}: P(\sigma) u \in H_{\mathrm{sc}}^{s, r+1}\right\} \rightarrow H_{\mathrm{sc}}^{s, r+1}
$$

is actually invertible; see [45, Section 5.4.8], and concretely Proposition 5.28 there. Note that the order is irrelevant away from the characteristic set, where the operator is elliptic. 
In order to do second microlocal analysis at the zero section, which is required for the $\sigma \rightarrow 0$ analysis as $P(\sigma)$ becomes degenerate from the sc-perspective at $\sigma=0$, we need to discuss first resolving the zero section at $\partial X$. Note that the weight (5.1) indeed becomes singular at the zero section; resolving the zero section removes this singularity.

From a slightly different perspective, the weight $r$ has to be monotone along the Hamilton flow of the principal symbol, and the Hamilton flow degenerates at the zero section since the principal symbol has a quadratic zero there, thus a resolution is needed. We recall that such a resolution, called a blowup, of a submanifold, here the boundary of the zero section, consists of replacing it by its inward pointing spherical normal bundle and naturally obtaining a smooth manifold with corners. Thus, one keeps track from which normal (i.e. modulo tangential) direction one is approaching the submanifold; correspondingly projective coordinates, like the ones discussed below, are usually particular easy to use.

Thus, we blow up the boundary $o_{\partial X}$ of the zero section $o$ in $\left.{ }^{{ }^{\mathrm{sc}}} T^{*} X:{ }^{\mathrm{sc}} T^{*} X ; o_{\partial X}\right]$; see Figure 4. Notice that near the interior of the front face, $x, y, \tau / x, \mu / x$ are coordinates. As one forms are written as

$$
\tau \frac{d x}{x^{2}}+\sum_{j} \mu_{j} \frac{d y_{j}}{x}=\frac{\tau}{x} \frac{d x}{x}+\sum_{j} \frac{\mu_{j}}{x} d y_{j},
$$

we have

$$
\tau_{\mathrm{b}}=\frac{\tau}{x}, \mu_{\mathrm{b}}=\frac{\mu}{x}
$$

in terms of the earlier discussed b-coordinates, i.e. the front face is exactly the b-cotangent bundle, ${ }^{\mathrm{b}} T^{*} X$, over $\partial X$.

On the other hand, near the corner given by the boundary of the front face, $x /\left(\tau^{2}+|\mu|^{2}\right)^{1 / 2}, \quad\left(\tau^{2}+|\mu|^{2}\right)^{1 / 2}, y$ plus homogeneous degree zero coordinates on the $(\tau, \mu)$-sphere (like $\tau /\left(\tau^{2}+|\mu|^{2}\right)^{1 / 2}$, etc.), give coordinates; note that $x /\left(\tau^{2}+\right.$ $\left.|\mu|^{2}\right)^{1 / 2}=\left(\tau_{\mathrm{b}}^{2}+\left|\mu_{\mathrm{b}}\right|^{2}\right)^{-1 / 2}$ is exactly the defining function of the lift of ${ }^{\mathrm{sc}} T_{\partial X}^{*} X$, while $\left(\tau^{2}+|\mu|^{2}\right)^{1 / 2}$ is the (local!) defining function of the front face, while the product of these two, $x$, is the total defining function of these two faces. The corner (i.e. the boundary of the front face) is identifiable with ${ }^{\mathrm{sc}} S^{*} X=\left({ }^{\mathrm{sc}} T^{*} X \backslash o\right) / \mathbb{R}^{+}$(the quotient being dilations in the fibers of a vector bundle), the sc-cosphere bundle, which is naturally identified in turn with ${ }^{\mathrm{b}} S^{*} X$, since the b- and sc- structures differ merely by a conformal factor. Indeed, the order function $r$ of (5.1) is a function on ${ }^{\mathrm{sc}} S^{*} X$ (thus on ${ }^{\mathrm{b}} S^{*} X$ ), thus on ${ }^{\mathrm{sc}} T^{*} X \backslash o$, and the blow-up allows the use of $r$ down to the zero section.

Near fiber infinity on $\overline{{ }^{\mathrm{sc}} T^{*}} X$, the blow-up does not change anything, so the defining function of fiber infinity is $\left(\tau^{2}+\mu^{2}\right)^{-1 / 2}=x^{-1}\left(\tau_{\mathrm{b}}^{2}+\mu_{\mathrm{b}}^{2}\right)^{-1 / 2}$, while that of the spatial boundary is $x$.

In order to actually do analysis, one needs pseudodifferential operators corresponding to this 2-microlocal resolution. Formally, one could say that one takes singular sc-symbols, namely ones that are conormal (or potentially classical) on the blown-up space, and quantizes these using the scattering quantization via local coordinate charts. The problem with this approach is that the contribution from the interior of the front face is necessarily global in nature (corresponds to the global normal operator in the b-setting), even modulo 'trivial' (rapidly decaying Schwartz kernel) terms, and thus it cannot literally make sense. This singular symbol approach is most common in the standard pseudodifferential setting, and requires 

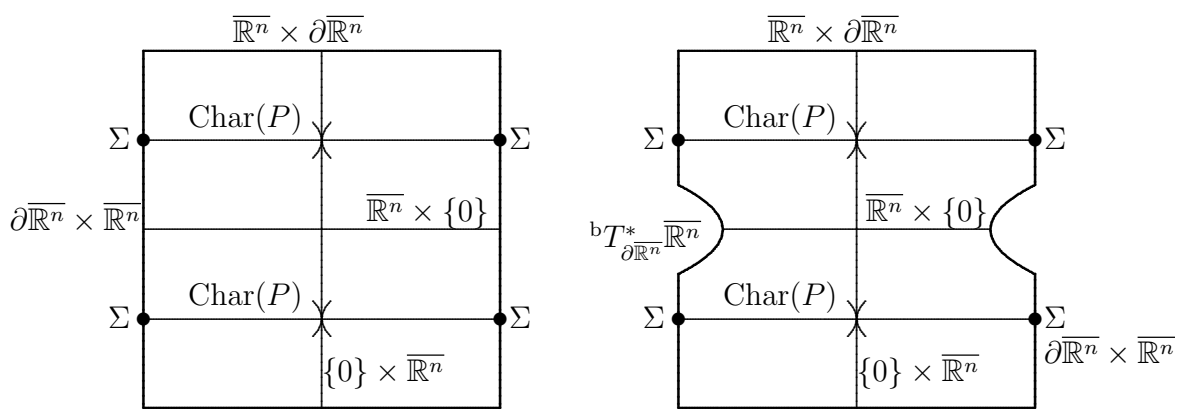

Figure 4. Second microlocalized Euclidean space $\mathbb{R}^{n}$. The left hand side is the fiber-compactified sc-cotangent bundle, $\overline{{ }^{s c} T^{*} \mathbb{R}^{n}}=$ $\overline{\mathbb{R}^{n}} \times \overline{\left(\mathbb{R}^{n}\right)^{*}}$, the right hand side is its blow-up at the boundary of the zero section. The (interior of the) front face of the blow-up, shown by the curved arcs, can be identified with ${ }^{b} T_{\partial \overline{\mathbb{R}^{n}}}^{*} \overline{\mathbb{R}^{n}}$. The characteristic set of $P(\sigma), \sigma \neq 0$, is also shown, both from the compactified perspective, as $\Sigma$, which is a subset of the boundary, and from the conic perspective, here conic in the base (i.e. the dilations are in the $\mathbb{R}_{z}^{n}$ factor), as Char $(P)$. The fiber of cotangent bundle over the origin, i.e. $\{0\} \times \overline{\left(\mathbb{R}^{n}\right)^{*}}$, is also indicated; this is only special from the conic (dilation) perspective, in which it is the analogue of the zero section in standard microlocal analysis.

significant effort to justify, see [6], and also [36] and [41] in the technically simpler semiclassical setting. In fact, even in the standard pseudodifferential setting it is conceptually easier to work with paired Lagrangian distributions, see [28, 19] and 10. for a recent treatment of these, though second microlocalization, which would correspond to the two Lagrangians being the conormal bundle of the diagonal on the one hand, and the product of the Lagrangian with itself ('primed', i.e. the covector sign reversed) on the other, has not been explicitly discussed using these (in [10] the singularities are lower order).
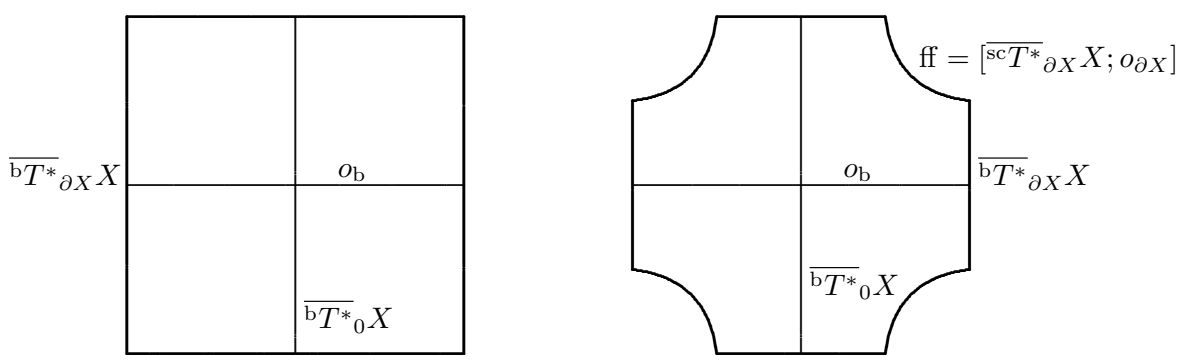

Figure 5. The second microlocal space, on the right, obtained by blowing up the corner of $\overline{{ }^{b} T^{*}} X$, shown on the left.

Thus, we consider instead the 'opposite' perspective, in which one starts with the b-cotangent bundle, and blows up its corner, i.e. fiber-infinity at the boundary, 
see Figure 5 A simple computation shows that the resulting space is naturally diffeomorphic to the previously discussed one:

Lemma 5.1. The identity map from the interior, $T^{*} X$, extends smoothly to an invertible map with smooth inverse, $\left[\overline{{ }^{\mathrm{b}} T^{*}} X ; \partial^{\mathrm{b} T^{*}} \partial X X\right] \rightarrow\left[\overline{{ }^{\mathrm{sc}} T^{*}} X ; o_{\partial X}\right]$.

Proof. We cover $\left[\overline{{ }^{\mathrm{b}} T^{*}} X ; \partial^{\overline{\mathrm{b}} T^{*}} \partial X X\right]$ and $\left[\overline{{ }^{\mathrm{sc}} T^{*}} X ; o_{\partial X}\right]$ by coordinate charts and show that the identity map restricted to the interior of these charts extends smoothly to the boundary in both directions. Concretely, we will have four different regions to deal with: one over $X^{\circ}$ (which is straightforward), and three near $\partial X$; for actual local coordinates we will then restrict to working over local coordinate charts $O$ in $\partial X$.

First of all, over $X^{\circ}$ both $\overline{{ }^{\mathrm{b}} T^{*}} X$ and $\overline{{ }^{\mathrm{sc}} T^{*}} X$, thus their blow ups (which are over $\partial X)$ are naturally identified with (in the same sense as above) $\overline{T^{*}} X$, i.e. the b- and sc-structures are irrelevant. Thus, it suffices to work near $\partial X$.

Next, in view of 5.2 , for $c>0$,

$$
\left\{\left(\tau^{2}+|\mu|^{2}\right)^{1 / 2}>c\right\}=\left\{x\left(\tau_{\mathrm{b}}^{2}+\left|\mu_{\mathrm{b}}\right|^{2}\right)^{1 / 2}>c\right\},
$$

and the former is a neighborhood of fiber infinity in $\overline{{ }^{\mathrm{sc}} T^{*} X}$, thus in $\left[\overline{{ }^{\mathrm{sc}} T^{*}} X ; o_{\partial X}\right]$ (as the blown up submanifold, $o_{\partial X}$, is disjoint from fiber infinity), while the latter is a neighborhood of the lift of fiber infinity to $\left[\overline{{ }^{\mathrm{b}} T^{*}} X ; \partial^{\mathrm{b} T^{*}} \partial X\right]$. If $y_{j}$ are local coordinates on a set $O$ in $\partial X$ then $x, y_{j},\left(\tau^{2}+|\mu|^{2}\right)^{-1 / 2}$ and spherical coordinates in $\left(\tau, \mu_{j}\right)$ are coordinates in the lift (inverse image under the blow-down map) of $\overline{\mathrm{sc}^{*}}{ }_{O} X$ in this region in $\left[\overline{{ }^{\mathrm{sc}} T^{*}} X ; o_{\partial X}\right]$, while $x, y_{j}, x^{-1}\left(\tau_{\mathrm{b}}^{2}+\left|\mu_{\mathrm{b}}\right|^{2}\right)^{-1 / 2}$ and spherical coordinates in $\left(\tau_{\mathrm{b}}, \mu_{\mathrm{b}}\right)$ are coordinates in the lift of ${ }^{\mathrm{b}} T^{*} O X$ in this region in $\left[\overline{{ }^{\mathrm{b}} T^{*}} X ; \partial^{\mathrm{b} T^{*}} \partial X X\right.$. Thus, $x^{-1}\left(\tau_{\mathrm{b}}^{2}+\left|\mu_{\mathrm{b}}\right|^{2}\right)^{-1 / 2}=\left(\tau^{2}+|\mu|^{2}\right)^{-1 / 2}$ and the spherical equivalence of $(\tau, \mu)$ and $\left(\tau_{\mathrm{b}}, \mu_{\mathrm{b}}\right)$ shows the equivalence of the two spaces (in the sense of smooth extension of the identity map in either direction) in this region.

Next, consider the region

$$
\left\{x^{-1}\left(\tau^{2}+|\mu|^{2}\right)^{1 / 2}<c\right\}=\left\{\left(\tau_{\mathrm{b}}^{2}+\left|\mu_{\mathrm{b}}\right|^{2}\right)^{1 / 2}<c\right\},
$$

$c>0$. This is on the one hand a neighborhood of a compact subset of the interior of the front face of $\left[\overline{{ }^{\mathrm{sc}} T^{*}} X ; o_{\partial X}\right]$, on the other hand a neighborhood of a compact subset of (the lift of) ${ }^{\mathrm{b}} T_{\partial X}^{*} X$ in $\left[\overline{\mathrm{b}} T^{*} X ; \partial^{\mathrm{b}} T^{*} \partial X X\right]$. Again if $y_{j}$ are local coordinates on a set $O$ in $\partial X$, then $x, y_{j}, \tau / x, \mu_{j} / x$ are (projective) coordinates in the lift of $\overline{\mathrm{sc}^{*}} O X$ in this region in $\left[{ }^{\left[{ }^{c} T^{*}\right.} X ; o_{\partial X}\right]$, while $x, y_{j}, \tau_{\mathrm{b}}, \mu_{\mathrm{b}}$ are coordinates in the lift of $\overline{{ }^{\mathrm{b}} T^{*}} O X$ in this region in $\left[\overline{{ }^{\mathrm{b}} T^{*}} X ; \partial^{\mathrm{b}} T^{*} \partial X X\right.$ (note that this blow-up does not affect this region), so by (5.2), the identity map extends to be smooth in this region.

Finally, consider the region

$$
\left\{c_{1} x<\left(\tau^{2}+|\mu|^{2}\right)^{1 / 2}<c_{2}\right\}=\left\{c_{1} x<x\left(\tau_{\mathrm{b}}^{2}+\left|\mu_{\mathrm{b}}\right|^{2}\right)^{1 / 2}<c_{2}\right\} .
$$

This is on the one hand a neighborhood of the boundary of the front face of $\left[\overline{{ }^{\mathrm{sc}} T^{*}} X ; o_{\partial X}\right]$ (as well as the new boundary of the lift of $\overline{\overline{\mathrm{sc}} T^{*}} \partial X X$ ), on the other hand a neighborhood of the boundary of the lift of $\overline{{ }^{\mathrm{b}} T^{*}} \partial X X$ to $\left[{ }^{\mathrm{b}} T^{*} X ; \partial^{\mathrm{b} T^{*}} \partial X X\right]$. If $y_{j}$ are local coordinates on a set $O$ in $\partial X$, then $x\left(\tau^{2}+|\mu|^{2}\right)^{-1 / 2}, y_{j},\left(\tau^{2}+|\mu|^{2}\right)^{1 / 2}$ and spherical coordinates in $\left(\tau, \mu_{j}\right)$ are coordinates in the lift of $\overline{{ }^{\mathrm{sc}} T^{*}} O X$ in this region in $\left[\overline{{ }^{c} T^{*}} X ; o_{\partial X}\right]$, while $x\left(\tau_{\mathrm{b}}^{2}+\left|\mu_{\mathrm{b}}\right|^{2}\right)^{1 / 2}, y_{j},\left(\tau_{\mathrm{b}}^{2}+\left|\mu_{\mathrm{b}}\right|^{2}\right)^{-1 / 2}$ and spherical coordinates in $\left(\tau_{\mathrm{b}}, \mu_{\mathrm{b}}\right)$ are coordinates in the lift of ${ }^{\mathrm{b}} T^{*} O X$ in this region in $\left[\overline{\mathrm{b}} T^{*} X ; \partial^{\mathrm{b}} T^{*} \partial X X\right]$, so by $\left(\right.$ with $\left(\tau^{2}+|\mu|^{2}\right)^{1 / 2}=x\left(\tau_{\mathrm{b}}^{2}+\left|\mu_{\mathrm{b}}\right|^{2}\right)^{1 / 2}$ and $x\left(\tau^{2}+|\mu|^{2}\right)^{-1 / 2}=\left(\tau_{\mathrm{b}}^{2}+\right.$ 
$\left.\left|\mu_{\mathrm{b}}\right|^{2}\right)^{-1 / 2}$ ) and the equivalence of the spherical variables, the identity map extends to be smooth in this region.

Since the regions listed (for various values of $c, c_{1}, c_{2}$ ) provide an open cover of $\left[\overline{{ }^{\mathrm{b}} T^{*}} X ; \partial^{\mathrm{b} T^{*}} \partial X X\right]$ as well as $\left[\overline{{ }^{\mathrm{sc}} T^{*}} X ; o_{\partial X}\right]$, the lemma follows.

The advantage of this perspective is that the blow-up is at fiber infinity, where symbolic improvements are available, and thus are easy to handle, i.e. the real work was done in defining $\Psi_{\mathrm{bc}}(X)$ and analyzing its properties, cf. the perspective of paired Lagrangian distributions in the case of standard microlocal analysis mentioned above.

Indeed, recall that on a manifold with corners $\mathcal{M}$ with boundary defining functions $\rho_{1}, \ldots, \rho_{k}$, the symbol space $S^{m_{1}, m_{2}, \ldots, m_{k}}(\mathcal{M})$ consists of $\mathcal{C}^{\infty}$ functions on the interior $\mathcal{M}^{\circ}$ which are in $\prod_{j=1}^{k} \rho_{j}^{-m_{j}} L^{\infty}$, and they remain so under iterated applications of $\mathcal{C}^{\infty}$ b-vector fields on $\mathcal{M}$, i.e. vector fields tangent to all boundary hypersurfaces. Suppose now that $\mathcal{M}$ is the radial compactification in the fibers of a rank $p$ vector bundle $\mathcal{Y}$ on a manifold with corners $\mathcal{X}$, where $\rho_{1}, \ldots, \rho_{k-1}$ are boundary defining functions of $\mathcal{X}$, and $\rho_{k}$ boundary defining function of fiber infinity, i.e. the new boundary hypersurface obtained via the radial compactification of the fibers. Thus, using local product decompositions given by local trivializations, with $\xi_{i}$ coordinates on the fibers of the local trivialization, $\rho_{k}$ can be taken to be locally equivalent to $|\xi|^{-1}$, with the norm being, say, the standard Euclidean norm. Then on the one hand this description of the symbol place applies directly on $\mathcal{M}$, and on the other hand the usual symbol space on $\mathcal{Y}$ is also defined via product decompositions given by local trivializations and requiring that iterated applications of combinations of b-vector fields on $\mathcal{X}$ and both linear and constant coefficient vector fields in the fibers (such as $\xi_{i} \partial_{\xi_{j}}$, resp. $\partial_{\xi_{j}}$ ), preserve estimates in $\prod_{j=1}^{k-1} \rho_{j}^{-m_{j}}|\xi|^{m_{k}} L^{\infty}=\prod_{j=1}^{k} \rho_{j}^{-m_{j}} L^{\infty}$. These two definitions are equivalent as is immediate in $|\xi|<C, C>0$ fixed, and in the region $|\xi|>C^{\prime}, C^{\prime}>0$, as the $\mathcal{C}^{\infty}\left(\overline{\mathbb{R}^{p}}\right)$ - (i.e. classical order 0 symbol) span of $\xi_{i} \partial_{\xi_{j}}$ is the same as $\mathcal{V}_{\mathrm{b}}\left(\overline{\mathbb{R}^{p}}\right)$ away from 0 (thus in this region), cf. the discussion in the introduction on symbols and b-vector fields on the radial compactification of Euclidean space.

Such a discussion applies in particular to ${ }^{{ }^{b} T^{*}} X$; thus the fully (in both differentiability and decay sense) conormal (as opposed to classical or polyhomogeneous) symbol space consists of $\mathcal{C}^{\infty}$ functions on the interior, ${ }^{\mathrm{b}} T_{X^{\circ}}^{*} X$, which satisfy a bound $|a| \leq C x^{-l}\left|\left(\tau_{\mathrm{b}}, \mu_{\mathrm{b}}\right)\right|^{m}$ stable under the iterative application of b-vector fields on $\overline{{ }^{\mathrm{b}} T^{*}} X$. In local coordinates, say where $\tau_{\mathrm{b}}>c\left|\mu_{\mathrm{b}}\right|(c>0)$, these are spanned by $x \partial_{x}, \partial_{y_{j}}, \tau_{\mathrm{b}} \partial_{\tau_{\mathrm{b}}}, \tau_{\mathrm{b}} \partial_{\left(\mu_{\mathrm{b}}\right)_{j}}$, here it is the fibers of the vector bundle in which the analogous compactification is taking place. This space is unchanged by this blow-up of the corner in the following sense:

Lemma 5.2. The pullback of $S^{m, l}\left(\overline{{ }^{\mathrm{b}} T^{*}} X\right)$ to $\left[\overline{{ }^{\mathrm{b}} T^{*}} X ; \partial^{\mathrm{b} T^{*}} \partial X X\right]$ by the blow-down map is $\left.S^{m, l, m+l}\left(\overline{\left[\mathrm{b} T^{*}\right.} X ; \partial^{\mathrm{b} T^{*}} \partial X X\right]\right)$, where the symbolic order at the lift of fiber infinity is $m$, at the lift of $x=0$ is $l$, while at the new front face the sum of these two orders, $m+l$.

Thus, for instance, symbols of order 0 in both senses pull back to symbols of order 0 in all three senses.

Proof. Local boundary defining functions of $\left[\overline{{ }^{\mathrm{b}} T^{*}} X ; \partial^{\mathrm{b} T^{*}} \partial X X\right]$ can be given as follows. In the complement of the lift of $x=0$ (and the b-zero section), which is the 
region (5.3) (if one takes the union over various $c>0), \rho_{\infty, \mathrm{loc}}=x^{-1}\left(\tau_{\mathrm{b}}^{2}+\left|\mu_{\mathrm{b}}\right|^{2}\right)^{-1 / 2}$ defines fiber infinity, $x$ defines the front face, so $\left|\left(\tau_{\mathrm{b}}, \mu_{\mathrm{b}}\right)\right|=\rho_{\infty, \text { loc }}^{-1} x^{-1}$, and

$$
x^{-l}\left|\left(\tau_{\mathrm{b}}, \mu_{\mathrm{b}}\right)\right|^{m} L^{\infty}=x^{-l-m} \rho_{\infty, \mathrm{loc}}^{-m} L^{\infty} .
$$

On the other hand, in the complement of the lift of fiber infinity (and, technically, away from the b-zero section, but the blow-up did not change the structure near the b-zero section), i.e. in the region (5.5) (but again as a union as $c_{1}, c_{2}$ vary) $\rho_{\mathrm{b}, \text { loc }}=x\left(\tau_{\mathrm{b}}^{2}+\left|\mu_{\mathrm{b}}\right|^{2}\right)^{1 / 2}$ defines the lift of $x=0$, and $\left(\tau_{\mathrm{b}}^{2}+\left|\mu_{\mathrm{b}}\right|^{2}\right)^{-1 / 2}$ defines the front face, so $x=\rho_{\mathrm{b}, \text { loc }}\left|\left(\tau_{\mathrm{b}}, \mu_{\mathrm{b}}\right)\right|^{-1}$, so

$$
x^{-l}\left|\left(\tau_{\mathrm{b}}, \mu_{\mathrm{b}}\right)\right|^{m} L^{\infty}=\rho_{\mathrm{b}, \text { loc }}^{-l}\left|\left(\tau_{\mathrm{b}}, \mu_{\mathrm{b}}\right)\right|^{l+m} L^{\infty} .
$$

In the intersection of these two regions, $x\left(\tau_{\mathrm{b}}^{2}+\left|\mu_{\mathrm{b}}\right|^{2}\right)^{1 / 2}$ is bounded both from above and from below by positive constants so these two spaces are indeed the same.

Finally, (lifts of ) b-vector fields on $\overline{\mathrm{b}} T^{*} X$ with coefficients in $\mathcal{C}^{\infty}\left(\left[\overline{\mathrm{b}} T^{*} X ; \partial^{\overline{\mathrm{b}} T^{*}} \partial X X\right]\right)$ span b-vector fields on $\left[\overline{\mathrm{b}} T^{*} X ; \partial^{\mathrm{b} T^{*}} \partial X X\right]$; this is a standard fact for blowing up a boundary face of a manifold with corners, which is very easily checked in local coordinates, using projective coordinates on the blown-up space.

Thus the iterative regularity statement for the symbols is unaffected by the blow-up process, proving the lemma.

An inspection in the various regions discussed above shows that

$$
\rho_{\infty}=\left(x^{2}\left(\tau_{\mathrm{b}}^{2}+\left|\mu_{\mathrm{b}}\right|^{2}\right)+1\right)^{-1 / 2}=\left(\tau^{2}+|\mu|^{2}+1\right)^{-1 / 2}
$$

is a global defining function of fiber infinity,

$$
\tilde{\rho}_{\mathrm{b}}=x\left(1+\tau_{\mathrm{b}}^{2}+\left|\mu_{\mathrm{b}}\right|^{2}\right)^{1 / 2}=\left(x^{2}+\tau^{2}+|\mu|^{2}\right)^{1 / 2}
$$

is a defining function of the lift of $x=0$ away from fiber infinity, and in the form

$$
\rho_{\mathrm{b}}=x\left(1+\tau_{\mathrm{b}}^{2}+\left|\mu_{\mathrm{b}}\right|^{2}\right)^{1 / 2} \rho_{\infty}=\left(x^{2}+\tau^{2}+|\mu|^{2}\right)^{1 / 2}\left(\tau^{2}+|\mu|^{2}+1\right)^{-1 / 2}
$$

even at fiber infinity.

Correspondingly:

Definition 5.3. Symbolic 2-microlocal pseudodifferential operators are defined as elements of $\Psi_{\mathrm{bc}}^{m, l}(X)$, where the subscript 'c' stands for the symbolic (conormal) behavior at both boundary hypersurfaces of $\overline{\mathrm{b}} T^{*} X$, and also for Schwartz kernels at the b-diagonal (corresponding, under the Fourier transform, to fiber infinity) and the b-front face.

Thus, at this level one cannot actually see any difference in the 2-microlocal vs. the b-framework, since the symbol spaces are the same. This definition assures that these conormal 2-microlocal operators form a *-algebra, with a full symbol calculus modulo $S^{-\infty, l}$, and a principal symbol calculus modulo $S^{m-1, l}$.

It is a simple matter of checking the composition rules, which in turn rely on left- and right- reductions of full symbols (amplitudes) to see that the subset given by quantizing classical symbols on $\left[\overline{\mathrm{b}} T^{*} X ; \partial^{\overline{\mathrm{b}} T^{*}} \partial X X\right]$ is also a *algebra. Note that these are certainly much larger than the pullback of classical symbols on $\overline{\mathrm{b}} T^{*} X$; for instance, a classical symbol supported near the interior of the front face $\left[\overline{\mathrm{b} T^{*}} X ; \partial^{\overline{\mathrm{b}} T^{*}} \partial X X\right]$ cannot be classical on $\overline{{ }^{\mathrm{b}} T^{*}} X$ unless it is of order $-\infty$ at the front face. As an illustration, if $m=l=0$, so classical symbols are smooth functions on 
$\left[\overline{\mathrm{b} T^{*}} X ; \partial^{\mathrm{b} T^{*}} \partial X X\right.$, even the principal symbol cannot be the pullback of a continuous function on ${ }^{\mathrm{b}} T^{*} X$ unless it vanishes. To see that this space is indeed closed under composition, we just need to consider, say, left reduction, i.e. eliminating dependence on the input variables of a joint amplitude (depending on both the input, primed, variables and the output, unprimed, variables), as composition ultimately follows from this. But this takes the form of an asymptotic sum, of terms of the form (constant multiples of $)\left.\left(-\partial_{t^{\prime}}\right)^{j} \partial_{y^{\prime}}^{\beta} \partial_{\tau_{\mathrm{b}}}^{j} \partial_{\mu_{\mathrm{b}}}^{\beta} a\left(t, y, t^{\prime}, y^{\prime}, \tau_{\mathrm{b}}, \mu_{\mathrm{b}}\right)\right|_{t^{\prime}=t, y^{\prime}=y}$, i.e. with an abuse of notation $\left.\left(x^{\prime} \partial_{x^{\prime}}\right)^{j} \partial_{y^{\prime}}^{\beta} \partial_{\tau_{\mathrm{b}}}^{j} \partial_{\mu_{\mathrm{b}}}^{\beta} a\left(x, y, x^{\prime}, y^{\prime}, \tau_{\mathrm{b}}, \mu_{\mathrm{b}}\right)\right|_{x^{\prime}=x, y^{\prime}=y}$, so as these differentiations preserve classicality even on the blown up space (since the differential operators lift to be smooth b-operators), this symbolic approximation of the left reduction of a classical amplitude is classical. Finally, the remainder term gives rise to a classical operator in $\Psi_{\mathrm{bc}}^{-\infty, l}$, i.e. one in $\Psi_{\mathrm{b}}^{-\infty, l}$, for which the composition property is easily checked explicitly.

The place where 2-microlocal considerations appear is that now one can microlocalize both at the lift of fiber infinity, of $\overline{{ }^{\mathrm{b}} T^{*}} X$, to $\left[\overline{{ }^{\mathrm{b}} T^{*}} X ; \partial^{\overline{\mathrm{b}} T^{*}} \partial X X\right.$, which is naturally identified with fiber infinity of $\overline{{ }^{s c} T^{*}} X$ and we simply refer to as fiber infinity below, and at new front face, which can naturally be identified with $\left[{ }^{\overline{{ }^{c}} T^{*}} \partial X X ; o_{\partial X}\right]$ on the one hand, and $\overline{\mathbb{R}^{+}} \times{ }^{\mathrm{b}} S_{\partial X}^{*} X$ on the other, and we simply refer to as the scattering face below. For instance:

Definition 5.4. One says that a pseudodifferential operator $A \in \Psi_{\mathrm{bc}}^{m, l}(X)$, written as the left quantization, say, of $a \in S^{m, l}$ modulo $\Psi_{\mathrm{bc}}^{-\infty, l}(X)$, is elliptic at a point $\alpha$ either at fiber infinity or at the scattering face if $\alpha$ has a neighborhood $O$ in

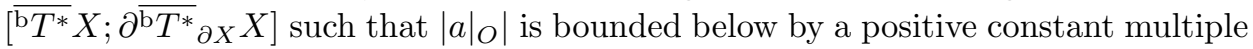
of the product of defining functions $\rho_{\infty}$ of fiber infinity raised to the power $-m$, $\rho_{\mathrm{sc}}$ of the scattering face raised to the power $-(m+l)$ and $\rho_{\mathrm{b}}$ of the b-face raised to the power $-l$.

Similarly, for such an $\alpha$ one says that $\alpha \notin \mathrm{WF}_{\mathrm{sc}, \mathrm{b}}^{\prime}(A)$ if $\alpha$ has a neighborhood $O$ in $\left[\overline{{ }^{\mathrm{b}} T^{*}} X ; \partial^{\mathrm{b} T^{*}} \partial X X\right]$ such that $\left.a\right|_{O}$ vanishes to infinite order at both of these hypersurfaces (inside $O$ ).

This makes these two boundary hypersurfaces the locus of the property of ellipticity and operator wave front set, and thus in the standard way also the locus of the wave front set of distributions, which is called the second microlocal sc-wave front set: in view of the above identifications this is a subset of the lift of the boundary hypersurfaces of $\overline{{ }^{s c} T^{*}} X$ to $\left[\overline{{ }^{s c} T^{*}} X ; o_{\partial X}\right]$. This refines the standard $\mathrm{WF}_{\mathrm{b}}$, which is at fiber infinity in $\overline{{ }^{\mathrm{b}} T^{*}} X$, usually identified with ${ }^{\mathrm{b}} S^{*} X$, or, via the $\mathbb{R}^{+}$-action in the fibers of ${ }^{\mathrm{b}} T^{*} X$, with conic subsets of ${ }^{\mathrm{b}} T^{*} X \backslash o_{\mathrm{b}}$.

In fact, this discussion indicates that it is useful to introduce a pseudodifferential operator space $\Psi_{\mathrm{sc}, \mathrm{b}}^{s, r, l}(X)$ with three orders corresponding to the three boundary hypersurfaces $\left[\overline{\mathrm{b}} T^{*} X ; \partial^{\mathrm{b}} T^{*} \partial X X\right]$ : the original two, giving the differential and bdecay orders, and the new one, at the front face, giving the sc-decay order; we order these as the fiber infinity order, the sc-decay order and finally the b-decay order. This thus arises from symbols in the class

$$
\rho_{\mathrm{b}}^{-l} \rho_{\mathrm{sc}}^{-r} \rho_{\infty}^{-s} S^{0,0}\left(\overline{{ }^{\mathrm{b}} T^{*}} X\right)=\rho_{\mathrm{b}}^{-l} \rho_{\mathrm{sc}}^{-r} \rho_{\infty}^{-s} S^{0,0,0}\left(\left[\overline{{ }^{\mathrm{b}} T^{*}} X ; \partial^{\overline{\mathrm{b}} T^{*}} \partial X X\right]\right)
$$


with $\rho_{\infty}$, resp. $\rho_{\mathrm{sc}}$, resp. $\rho_{\mathrm{b}}$ the defining functions of the lift of fiber infinity, the scattering face, and the lift of $x=0$, respectively. This is a subset of

$$
x^{-l}\left(\tau_{\mathrm{b}}^{2}+\left|\mu_{\mathrm{b}}\right|^{2}\right)^{(s+\max (r-(s+l), 0)) / 2} S^{0,0}\left(\overline{\mathrm{b}} T^{*} X\right),
$$

for, as discussed before, the symbol space is insensitive to the blowup, and the weights give symbolic orders $l$ at the the lift of $x=0, s+\max (r-(s+l), 0) \geq s$ at fiber infinity, and $l+s+\max (r-(s+l), 0) \geq r$ at the scattering face. Thus,

$$
\Psi_{\mathrm{sc}, \mathrm{b}}^{s, r, l}(X) \subset \Psi_{\mathrm{bc}}^{s+\max (r-(s+l), 0), l}(X),
$$

and membership in this smaller subspace is characterized purely by symbolic properties, namely finite order vanishing conditions within the class, concretely of order $\max (r-(s+l), 0)$ at fiber infinity and $l+s-r+\max (r-(s+l), 0)=\max ((s+l)-r, 0)$ at the scattering face. Therefore, $\Psi_{\mathrm{sc}, \mathrm{b}}^{s, r, l}(X)$ can be easily seen to be invariant under composition and adjoints by the left-reduction formula discussed above. Notice that one has

$$
\Psi_{\mathrm{bc}}^{m, l}(X)=\Psi_{\mathrm{sc}, \mathrm{b}}^{m, m+l, l}(X) .
$$

Alternatively, though we do not give details here, this class can be characterized by considering operators of the form $A=A_{1}+A_{2}$ where $A_{1} \in \Psi_{\mathrm{bc}}^{s, r-s}(X)$ and $A_{2} \in$ $\Psi_{\mathrm{bc}}^{r-l, l}$ and the amplitude of $A_{1}$ (i.e. when $A_{1}$ is written as a, say, left quantization) is microlocalized away from the lift of $\overline{{ }^{\mathrm{b}} T^{*}} \partial X X$, while, modulo $\Psi_{\mathrm{bc}}^{-\infty, l}(X)$, the amplitude of $A_{2}$ is microlocalized away from fiber infinity, i.e. the lift of $\partial^{\overline{\mathrm{b}} T^{*}} X$. A priori it is not clear that this is a well-behaved space of operators under composition. This is made clear by using a decomposition $A_{1}+A_{2}+A_{3}$ where $A_{1}, A_{2}$ as above, but with amplitudes supported in a pre-specified (small) neighborhood of fiber infinity, resp. the lift of $\overline{{ }^{b} T^{*}} \partial X X$, while

$$
A_{3} \in \Psi_{\mathrm{bc}}^{s, r-s}(X) \cap \Psi_{\mathrm{bc}}^{r-l, l}(X), \text { indeed } A_{3} \in \Psi_{\mathrm{scc}}^{-\infty, r}(X)
$$

has amplitude microlocalized away from both of these boundary hypersurfaces, i.e. the essential support of the amplitude only intersecting the interior of the front face of the blow up (the sc-face). Thus, $A_{3}$ can be combined with either of the other two terms for the use of standard $\Psi_{\mathrm{bc}}$ composition results, while $A_{1} A_{2} \in \Psi_{\mathrm{bc}}^{-\infty,-\infty}(X)$ in fact as can be seen by first of all noting that the symbolic expansion is trivial due to the disjoint support (in the compactified sense) of the two amplitudes, which implies $A_{1} A_{2}, A_{2} A_{1} \in \Psi_{\mathrm{bc}}^{-\infty, r-s+l}(X)$, but in addition in fact the error term of the asymptotic expansions, given by an integral Taylor series remainder term also possesses additional decay properties. (The modulo $\Psi_{\mathrm{bc}}^{-\infty, l}(X)$ part of $A_{2}$ is easily seen to be harmless.)

The second microlocal Sobolev spaces, $H_{\mathrm{sc}, \mathrm{b}}^{s, r, l}(X)$, are then based on these pseudodifferential spaces, e.g. if all indices are $\geq 0$, the space simply consists of $u \in L^{2}$ such that for all $A \in \Psi_{\mathrm{sc}, \mathrm{b}}^{s, r, l}$ (or simply for one elliptic one) $A u \in L^{2}$. We refer to [45, Section 5.3.9] for a detailed discussion. Thus,

$$
H_{\mathrm{b}}^{\tilde{r}, l}=H_{\mathrm{sc}, \mathrm{b}}^{\tilde{r}, \tilde{r}+l, l}
$$

Also, sc-microlocally away from the zero section at $\partial X$, i.e. away from the lift of $x=0$ in the b-blow up perspective, $H_{\mathrm{sc}, \mathrm{b}}^{s, r, l}$ is just $H_{\mathrm{sc}}^{s, r}$, and the estimate below is just the estimate leading to the (strictly positive spectral parameter) limiting absorption principle in the scattering setting. However, the inclusion of $H_{\mathrm{sc}, \mathrm{b}}^{s, r, l}$ into $H_{\mathrm{sc}, \mathrm{b}}^{s^{\prime}, r^{\prime}, l}$ is no 
longer compact since there is no improvement of the weight sc-microlocally at the zero section at $\partial X$. Notice also that elements of $\Psi_{\mathrm{sc}, \mathrm{b}}^{\tilde{s}, \tilde{l}}(X)$ give continuous linear $\operatorname{maps} H_{\mathrm{sc}, \mathrm{b}}^{s, r, l} \rightarrow H_{\mathrm{sc}, \mathrm{b}}^{s-\tilde{s}, r-\tilde{r}, l-\tilde{l}}$.

The symbolic estimate, cf. Section 3 is then:

Proposition 5.5. Suppose $S \subset[0, \infty)$ compact. With $s, r, l$ as above, with $r \in$ $\mathcal{C}^{\infty}\left({ }^{\mathrm{sc}} S^{*} X\right)=\mathcal{C}^{\infty}\left({ }^{\mathrm{b}} S^{*} X\right)$ monotone along the $\mathrm{H}_{p}$-flow, satisfying the threshold inequalities relative to $-\frac{1}{2}, s^{\prime}, r^{\prime}$ arbitrary, there exists $C>0$ such that

$$
\|u\|_{H_{\mathrm{sc}, \mathrm{b}}^{s, r, l}} \leq C\left(\|P(\sigma) u\|_{H_{\mathrm{sc}, \mathrm{b}}^{s-2, r+1, l+2}}+\|u\|_{H_{\mathrm{sc}, \mathrm{b}}^{s^{\prime}, r^{\prime}, l}}\right), \quad \sigma \in S
$$

provided $u \in H_{\mathrm{sc}, \mathrm{b}}^{s^{\prime \prime}, r^{\prime \prime}, l}$ for some $s^{\prime \prime}, r^{\prime \prime}$ with $r^{\prime \prime}>-\frac{1}{2}$ at the high regularity radial point (for $r$ ).

Proof. This estimate again arises from the 'same' positive commutator argument as in the b-algebra, with some slight modifications in the present second microlocalized scattering algebra setting, combined with an elliptic estimate.

Indeed, for $\sigma$ in a fixed compact set, the operator is elliptic near fiber infinity, so if $\tilde{\varphi}$ is supported away from 0 , identically 1 near infinity, and $B$ has principal symbol $\tilde{\varphi}=\tilde{\varphi}\left(\tau^{2}+|\mu|^{2}\right)$ with wave front set on $\operatorname{supp} \tilde{\varphi}$ we have elliptic estimates of the sort

$$
\|\tilde{B} u\|_{H_{\mathrm{sc}, \mathrm{b}}^{s, r, l}} \leq C\left(\|P(\sigma) u\|_{H_{\mathrm{sc}, \mathrm{b}}^{s-2, r, l}}+\|u\|_{H_{\mathrm{sc}, \mathrm{b}}^{s^{\prime}, r^{\prime}, l^{\prime}}}\right)
$$

for any $s^{\prime}, r^{\prime}, l^{\prime}$. (Notice that this in particular replaces the elliptic estimate 3.14) in Section 3 .)

On the other hand, away from fiber infinity, as $\mathrm{H}_{p}\left(\tau^{2}+|\mu|^{2}\right)=0$ at $\partial X$ (and indeed one could simply use $p$ in place of $\tau^{2}+|\mu|^{2}$, and then not just at $\partial X$ ), we can simply use a cutoff in $\tau^{2}+|\mu|^{2}$, namely taking a function $\varphi$ identically 1 near 0 , of compact support, and thus replace $(3.4$ by

$$
a=x^{-l-1}\left(\tau_{\mathrm{b}}^{2}+\left|\mu_{\mathrm{b}}\right|^{2}\right)^{(\tilde{r}-1 / 2) / 2} \varphi\left(\tau^{2}+|\mu|^{2}\right) \psi(x),
$$

where $\tilde{r}=r-l$ satisfies the inequalities for $\tilde{r}$ discussed in Section 3 , adding also a regularizer as in (3.11). Thus, we conclude, with $B$ as discussed around $(3.13$ but with a factor $\varphi\left(\tau^{2}+|\mu|^{2}\right)$ added to $b$, and with $\tilde{s}, \tilde{s}^{\prime}$ arbitrary (and irrelevant), that

$$
\|B u\|_{H_{\mathrm{sc}, \mathrm{b}}^{\tilde{s}, r, l}} \leq C\left(\|P(\sigma) u\|_{H_{\mathrm{sc}, \mathrm{b}}^{\tilde{s}^{\prime}, r+1, l+2}}+\|u\|_{H_{\mathrm{sc}, \mathrm{b}}^{s^{\prime}, r^{\prime}, l}}\right) .
$$

Combining these two, with $\varphi=1-\tilde{\varphi}$ and $B=\operatorname{Id}-\tilde{B}$, using

$$
\|u\|_{H_{\mathrm{sc}, \mathrm{b}}^{s, r, l}} \leq\|\tilde{B} u\|_{H_{\mathrm{sc}, \mathrm{b}}^{s, r, l}}+\|B u\|_{H_{\mathrm{sc}, \mathrm{b}}^{s, r, l}}
$$

proves the proposition.

Since microlocally near the scattering 0 -section the second microlocal Sobolev space is just the b-Sobolev space, the previous normal operator estimate, (4.7),

$$
\|v\|_{H_{\mathrm{b}}^{\tilde{r}, l}} \leq C\|\tilde{N}(P(\sigma)) v\|_{H_{\mathrm{b}}^{\tilde{r}^{\prime}, l+2}}
$$

can be used to improve (weaken the norm on) the error term on the right hand side of (5.6). However, to avoid the technicalities which lengthened the proof of Proposition 4.1, we use the result of that proposition directly.

One can then proceed as in Section 4 to prove an analogue (and extension, since we use this proposition in the proof below) of Proposition 4.1 . 
Proposition 5.6. Let $S \subset[0, \infty)$ compact.

With $s, r, l$ as above, with $r \in \mathcal{C}^{\infty}\left({ }^{\mathrm{sc}} S^{*} X\right)=\mathcal{C}^{\infty}\left({ }^{\mathrm{b}} S^{*} X\right)$ monotone along the $\mathrm{H}_{p}$-flow, satisfying the threshold inequalities relative to $-\frac{1}{2}, s^{\prime}, r^{\prime}$ arbitrary, there exists $C$ such that we have the estimate

$$
\|u\|_{H_{\mathrm{sc}, \mathrm{b}}^{s, r, l}} \leq C\left(\|P(\sigma) u\|_{H_{\mathrm{sc}, \mathrm{b}}^{s-2, r+1, l+2}}+\|u\|_{H_{\mathrm{sc}, \mathrm{b}}^{s^{\prime}, r^{\prime}, l-\delta}}\right), \sigma \in S,
$$

provided $u \in H_{\mathrm{sc}, \mathrm{b}^{\prime \prime}, l}^{s^{\prime \prime}}$ for some $s^{\prime \prime}, r^{\prime \prime}$ with $r^{\prime \prime}>-\frac{1}{2}$ at the high regularity radial point (for $r$ ).

Proof. First we remark that if $r=(s-1)+l$, and hence $r+1=(s-2)+(l+2)=$ $s+l$, then the slightly weakened version of (5.11) is equivalent to the estimate of Proposition 4.1, since in the second microlocal notation that states

$$
\begin{aligned}
&\|u\|_{H_{\mathrm{sc}, \mathrm{b}}^{s-1, s+l-1, l}} \sim\|u\|_{H_{\mathrm{b}}^{s-1, l}} \leq C\left(\|P(\sigma) u\|_{H_{\mathrm{b}}^{s-2, l+2}}+\|u\|_{H_{\mathrm{b}}^{r^{\prime}, l-\delta}}\right) \\
& \sim C\left(\|P(\sigma) u\|_{H_{\mathrm{sc}, \mathrm{b}}^{s-2, s+l, l+2}}+\|u\|_{H_{\mathrm{sc}, \mathrm{b}}^{r^{\prime}, r^{\prime}+l-\delta, l-\delta}}\right)
\end{aligned}
$$

with $r^{\prime}$ arbitrary (but membership of $u$ in a stronger space required). The only sense in which this is weaker than (5.11) is that the latter is an elliptic lossless estimate in the first order, $s$. This is easily remedied by using Proposition 5.5 . which in combination with 5.12 gives

$$
\begin{aligned}
\|u\|_{H_{\mathrm{sc}, \mathrm{b}}^{s, r, l}} & \leq C\left(\|P(\sigma) u\|_{H_{\mathrm{sc}, \mathrm{b}}^{s-2, r+1, l+2}}+\|u\|_{H_{\mathrm{sc}, \mathrm{b}}^{s-1, r, l}}\right) \\
& \leq C^{\prime}\left(\|P(\sigma) u\|_{H_{\mathrm{sc}, \mathrm{b}}^{s-2, r+1, l+2}}+\|u\|_{H_{\mathrm{sc}, \mathrm{b}}^{r^{\prime}, r^{\prime}+l-\delta, l-\delta}}\right),
\end{aligned}
$$

which is the estimate of the present proposition in this special case as $r^{\prime}$ is arbitrary.

Indeed, the same argument works if $r \leq(s-1)+l$, i.e. $s \geq r-l+1$, for first let $\tilde{s}=r-l+1 \leq s$, and apply 5.12 with $s$ replaced by $\tilde{s}$ to get

$$
\|u\|_{H_{\mathrm{sc}, \mathrm{b}}^{\tilde{s}-1, \tilde{s}+l-1, l}} \leq C\left(\|P(\sigma) u\|_{H_{\mathrm{sc}, \mathrm{b}}^{\tilde{s}-2, \tilde{s}+l, l+2}}+\|u\|_{H_{\mathrm{sc}, \mathrm{b}}^{r^{\prime}, r^{\prime}+l-\delta, l-\delta}}\right)
$$

and now by Proposition 5.5 we have

$$
\begin{aligned}
& \|u\|_{H_{\mathrm{sc}, \mathrm{b}}^{s, r, l}} \leq C\left(\|P(\sigma) u\|_{H_{\mathrm{sc}, \mathrm{b}}^{s-2, r+1, l+2}}+\|u\|_{H_{\mathrm{sc}, \mathrm{b}}^{\tilde{s}-1, r, l}}\right) \\
& \leq C^{\prime}\left(\|P(\sigma) u\|_{H_{\mathrm{sc}, \mathrm{b}}^{s-2, r+1, l+2}}+\|P(\sigma) u\|_{H_{\mathrm{sc}, \mathrm{s}}^{\tilde{s}-2, r+1, l+2}}+\|u\|_{H_{\mathrm{sc}, \mathrm{b}}^{r^{\prime}, r^{\prime}+l-\delta, l-\delta}}\right) \\
& \leq C^{\prime \prime}\left(\|P(\sigma) u\|_{H_{\mathrm{sc}, \mathrm{b}}^{s-2, r+1, l+2}}+\|u\|_{H_{\mathrm{sc}, \mathrm{b}}^{r^{\prime}, r^{\prime}+l-\delta, l-\delta}}\right),
\end{aligned}
$$

proving (5.11) under the additional assumption $s \geq r-l+1$.

Now we dualize the argument, much as in the proof of Proposition 4.1. Namely, first re-write the existing estimate, adding a subscript + to denote that the order $r$ is monotone increasing along the Hamilton flow; the estimate then holds if $s_{+} \geq$ $r_{+}-l_{+}+1$. But then the analogous estimate also holds with the monotonicity relative to the Hamilton flow reversed, and with $P(\sigma)$ (potentially) replaced by $P(\sigma)^{*}$, namely

$$
\|u\|_{H_{\mathrm{sc}, \mathrm{b}}^{s_{-}, r_{-}, l_{-}}} \leq C\left(\left\|P(\sigma)^{*} u\right\|_{H_{\mathrm{sc}, \mathrm{b}}^{s_{-}-2, r_{-}+1, l_{-}+2}}+\|u\|_{H_{\mathrm{sc}, \mathrm{b}}^{r^{\prime}, r_{-}^{\prime}+l-\delta, l_{-}-\delta}}\right),
$$

valid if $s_{-} \geq r_{-}-l_{-}+1$. We now apply this with $r_{-}=-\left(1+r_{+}\right), l_{-}=-\left(l_{+}+2\right)$, since we need this dual estimate with $H_{\mathrm{sc}, \mathrm{b}}^{s_{-}, r_{-}, l}=\left(H_{\mathrm{sc}, \mathrm{b}}^{s_{+}-2, r_{+}+1, l_{+}+2}\right)^{*}$. The desired choice of $s_{-}$would be $2-s_{+}$, but as $s_{+} \geq r_{+}-l_{+}+1,2-s_{+} \leq-r_{+}+l_{+}-1=r_{-}-l_{-}$ which cannot be satisfied if $s_{-}=2-s_{+}$and $s_{-} \geq r_{-}-l_{-}+1$. Thus, we choose 
$s_{-}=r_{-}-l_{-}+1=-1-r_{+}+l_{+}+2+1=3-\left(r_{+}-l_{+}+1\right) \geq 2-s_{+}$for applying (5.14), which gives us a version of the dual estimate we actually want

$$
\|u\|_{H_{\mathrm{sc}, \mathrm{b}}^{2-s_{+},-1-r_{+},-l_{+}-2}} \leq C\left(\left\|P(\sigma)^{*} u\right\|_{H_{\mathrm{sc}, \mathrm{b}}^{-s_{+},-r_{+},-l_{+}}}+\|u\|_{H_{\mathrm{sc}, \mathrm{b}}^{r^{\prime}, r_{-}^{\prime}+l-\delta, l_{-}-\delta}}\right),
$$

with the proviso that the norms in (5.14) are stronger than in this estimate in the sc-differential sense. However, we argue as in the proof of Proposition 4.1. Namely, ignoring finite dimensional solvability obstacles which are handled exactly in the same way as in Proposition 4.1 the estimate (5.14 lets us solve $P(\sigma) u=f$ when $f \in H_{\mathrm{sc}, \mathrm{b}}^{-s_{-},-r_{-},-l_{-}}=H_{\mathrm{sc}, \mathrm{b}}^{\left(r_{+}-l_{+}+1\right)-3, r_{+}+1, l_{+}+2}$, with the resulting $u \in H_{\mathrm{sc}, \mathrm{b}}^{2-s_{-},-1-r_{-},-l_{-}-2}=H_{\mathrm{sc}, \mathrm{b}}^{\left(r_{+}-l_{+}+1\right)-1, r_{+}, l_{+}}$. Now, if $f$ has the additional regularity $f \in H_{\mathrm{sc}, \mathrm{b}}^{s_{+}-2, r_{+}+1, l_{+}+2}$ (note that $s_{+}-2 \geq\left(r_{+}-l_{+}+1\right)-3$ ), the elliptic estimates (or directly Proposition 5.5 imply that $u \in H_{\mathrm{sc}, \mathrm{b}}^{s_{+}, r_{+}, l_{+}}$, with an estimate for $\|u\|_{H_{\mathrm{sc}, \mathrm{b}}^{s_{+}, r_{+}, l_{+}}}$, which via a pairing argument as before 4.15 also gives the adjoint estimate $(5.15)$.

We finally eliminate the restriction on $s_{+}$by noting that reversing the role of $P(\sigma)$ and $P(\sigma)^{*}$ (i.e. obtaining the original estimate for $P(\sigma)^{*}$, thus dual estimate (5.15) for $\left(P(\sigma)^{*}\right)^{*}=P(\sigma)$ in place of $P(\sigma)^{*}$, gives the desired estimate when the orders satisfy $2-s_{+} \leq 2-\left(r_{+}-l_{+}+1\right) \leq\left(-1-r_{+}\right)-\left(-l_{+}-2\right)+1$, which, when relabeling $2-s_{+}$as $s_{-},-1-r_{+}$as $r_{-},-l_{+}-2$ as $l_{-}$, is exactly the desired estimate for (a bigger range than) $s_{-} \leq r_{-}-l_{-}+1$. Since the estimate for monotone weights with either direction of monotonicity are completely analogous, this completes the proof of the proposition.

Again, this has the errorless consequence if $P(0)$ has trivial nullspace, since the error term can be dropped:

Theorem 5.7. Suppose that $s, r, l$ are as in Proposition 5.6. Suppose also that $P(0)$ has trivial nullspace on $H_{\mathrm{sc}, \mathrm{b}}^{\infty, \infty, l}=H_{\mathrm{b}}^{\infty, l}$. Then there are $\sigma_{0}>0, C>0$ such that for $\sigma \in\left[0, \sigma_{0}\right]$,

has uniform bounds:

$$
P(\sigma)^{-1}: H_{\mathrm{sc}, \mathrm{b}}^{s-2, r+1, l+2} \rightarrow H_{\mathrm{sc}, \mathrm{b}}^{s, r, l}
$$

$$
\left\|P(\sigma)^{-1} f\right\|_{H_{\mathrm{sc}, \mathrm{b}}^{s-2, r+1, l+2}} \leq C\|f\|_{H_{\mathrm{sc}, \mathrm{b}}^{s, r, l}}, \quad \sigma \in S .
$$

In particular, this proves Corollary 1.10

\section{The KerR SETting}

In this section we concentrate on the analytic aspects of the Kerr setting that extend those of the setting of the earlier parts of this this paper, which describe the Kerr wave operator near the Minkowski (or Euclidean) end. In order to deal with the full Kerr setting, given the work already done in this paper, we need to deal with a neighborhood of the event horizon. All the relevant features there were handled by the microlocal Fredholm analysis of [40, though here we frame the operator as one equipped with a Cauchy hypersurface inside the black hole, rather than complex absorption, following [24. One then considers a manifold with boundary $X$ as before, except that $X$ has, in addition to the asymptotically Euclidean boundary, which we denote $\partial_{+} X$, an 'artificial boundary' $\partial_{-} X$, given by $\mu=\mu_{0}, \mu_{0}<0$, where $\mu$ is a smooth function on $X$, with $\mu-\mu_{0}$ a defining function 
of $\partial_{-} X$; this acts as a Cauchy hypersurface below. We still use $x=r^{-1}$ to denote a defining function of $\partial_{+} X$, and assume that near $\partial_{+} X$ the same structure as before holds. In the actual Kerr spacetime, we have $x=r^{-1}, \mu=1-\frac{2 m}{r}, m$ the mass of the black hole.

As $\partial_{-} X$ is not considered a 'real' boundary, below the notation Diff ${ }_{\mathrm{b}}$, etc., refers to the b-structure at $\partial_{+} X$, and the standard space of differential operators at $\partial_{-} X$. We then consider

$$
P(\sigma)=P(0)+\sigma Q-\sigma^{2}, \quad P(0) \in x^{2} \operatorname{Diff}_{\mathrm{b}}^{2}(X), Q \in S^{-2-\delta} \operatorname{Diff}_{\mathrm{b}}^{1}(X)
$$

$P(\sigma)$ is symmetric for $\sigma$ real (so $P(0)$ and $Q$ symmetric; here we explicitly make $Q$ independent of $\sigma$ ), and $P(0)-\Delta_{g}$ in $S^{-2-\delta} \operatorname{Diff}_{\mathrm{b}}^{2}(X)$ near $\partial_{+} X$, so in particular $P(0)$ is elliptic in $\Psi_{\mathrm{bc}}^{2,0}$ in $x<x_{0}$ for some $x_{0}>0$, while $P(0)$ is a wave operator near $\partial_{-} X$ with $\mu$ timelike satisfying microlocal hypotheses on the structure of the Hamilton flow that we describe next.

We remark that for the actual Kerr wave operator $\square$, for suitable positive $\alpha \in$ $\mathcal{C}^{\infty}(X)$, with $\alpha-1 \in x \mathcal{C}^{\infty}(X), \alpha \square$ is of this form $(\alpha \sim 1-2 m / r=1-2 m x$ near $x=0, m$ the mass of the black hole, $\sim$ in the sense that the difference is $O\left(x^{2}\right)$ ), which then remains formally self-adjoint relative to $\alpha^{-1}$ times the metric volume density. This form is the consequence of a simple computation near $x=0$ using Boyer-Lindquist coordinates (which need to be modified away from $x=0$ to deal with the horizon, much as in the Kerr-de Sitter setting) in which the dual metric is

$$
G=-\rho^{-2}\left(\Delta_{r} \partial_{r}^{2}+\frac{1}{\sin ^{2} \theta}\left(a \sin ^{2} \theta \partial_{t}+\partial_{\phi}\right)^{2}+\partial_{\theta}^{2}-\frac{1}{\Delta_{r}}\left(\left(r^{2}+a^{2}\right) \partial_{t}+a \partial_{\phi}\right)^{2}\right)
$$

with $m, a$ constants, $0 \leq|a|<m$,

$$
\rho^{2}=r^{2}+a^{2} \cos ^{2} \theta, \Delta_{r}=r^{2}+a^{2}-2 m r .
$$

Indeed, expanding the squares yields, with $h_{S^{2}}^{-1}$ the spherical dual metric, $k_{S}$ a smooth 2-tensor family (in $r^{-1}$ ) on the sphere,

$$
\begin{gathered}
\left((1-2 m / r)^{-1}+O\left(r^{-2}\right)\right) \partial_{t}^{2}-\left(1-2 m / r+O\left(r^{-2}\right) \partial_{r}^{2}\right. \\
-r^{-2}\left(h_{S^{2}}^{-1}+O\left(r^{-1}\right) k_{S}\right)-O\left(r^{-3}\right) \partial_{t} \partial_{\phi},
\end{gathered}
$$

where the cancellation between the two $\partial_{t} \partial_{\phi}$ cross terms,

$$
\left(r^{2}+a^{2} \cos ^{2} \theta\right)^{-1}\left(2 a \partial_{t} \partial_{\phi}-2 \Delta_{r}^{-1} a\left(r^{2}+a^{2}\right) \partial_{t} \partial_{\phi}\right),
$$

with $\Delta_{r}^{-1}\left(r^{2}+a^{2}\right)=1+O(r)$ giving the cancellation, is crucial in the decay in $O\left(r^{-3}\right) \partial_{t} \partial_{\phi}$ that we use here. Moreover, in the actual wave operator, the $t$ translation invariance means that there are no first or zeroth order terms arising from the non-decaying $\left.(1-2 m / r)^{-1}+O\left(r^{-2}\right)\right) \partial_{t}^{2}$ term of the dual metric. Thus, with $\alpha$ being the reciprocal of the coefficient of $\partial_{t}^{2}$ (i.e. the squared metric length of $d t$ ), this gives the desired form near $\partial_{+} X$.

The description of the hypotheses away from $\partial_{+} X$ follows [40, Section 2.2] closely (with some minor notational changes); indeed away from $\partial_{+} X$ behaves much like a Kerr-de Sitter metric. In order to deal with the structure of the operator away from $\partial_{+} X$ we also assume that the principal symbol $p$ of $P(0)$ has a non-degenerate zero set $\Sigma=\Sigma_{+} \cup \Sigma_{-}$with the subscript denoting two connected components (i.e. $d p$ does not vanish when $p$ does), and has non-degenerate, in the sense of [40, Section 2.2], generalized radial sets $\Lambda_{ \pm}^{-}$which are submanifolds of ${ }^{\mathrm{b}} T_{X^{\circ}}^{*} X$ (of course as $\Lambda^{-}$lies over the interior of $X$, there is no distinction between ${ }^{\mathrm{b}} T^{*}$ and $T^{*}$ near $\Lambda^{-}$, i.e. the 
' $\mathrm{b}$ ' nature is irrelevant) (in the actual Kerr space these would be the two halves of $N^{*} Y$, where $Y \subset X$ is the event horizon), and $\Lambda_{ \pm}^{-}$are (normal) sources $(-) /$sinks (+) for $\mathrm{H}_{p}$ within ${ }^{\mathrm{b}} T^{*} X$, with $\Lambda_{ \pm}^{-} \subset \Sigma_{\mp}$, with the superscript standing for the black hole end. We write $L^{-}$for the image of $\Lambda^{-}$, with the appropriate subscripts, in the cosphere bundle ${ }^{\mathrm{b}} S^{*} X$. The subprincipal symbol of $P(\sigma)$ at $L_{ \pm}^{-}$also plays a role below; we assume that, with principal symbols considered as homogeneous functions (of degree 1 ) on ${ }^{\mathrm{b}} T^{*} X \backslash o$, and $\rho_{\infty}$ being a defining function of fiber infinity (with asymptotic degree -1 homogeneity, and with the particular choice not playing any role)

$$
\left.\sigma_{1}\left(\frac{1}{2 i}\left(P(\sigma)-P(\sigma)^{*}\right)\right)\right|_{\Lambda_{ \pm}^{-}}=(\operatorname{Im} \sigma) \sigma_{1}(Q)=\left.\kappa(\operatorname{Im} \sigma) \rho_{\infty}^{-1} \mathrm{H}_{p} \rho_{\infty}\right|_{\Lambda_{ \pm}^{-}}
$$

for a function $\kappa>0$; for notational simplicity we take $\kappa$ constant.

Such a Cauchy hypersurface plus radial point plus the b-infinity setting is called non-trapping if (null)-bicharacteristics of $p$ have the following behavior:

(i) All bicharacteristics in $\Sigma_{+} \backslash L_{-}^{-}$tend to $L_{-}^{-}$in the backward direction and to $\partial_{-} X$ (meaning ${ }^{\mathrm{b}} T_{\partial_{-} X}^{*} X$ ) in the forward direction.

(ii) All bicharacteristics in $\Sigma_{-} \backslash L_{+}^{-}$tend to $L_{+}^{-}$in the forward direction and to $\partial_{-} X$ in the backward direction.

Thus, in $\Sigma_{+}, L^{-}$can only be reached in the backward direction and $\partial_{-} X$ in the forward direction; in $\Sigma_{-}$forward and backward are reversed. (Microlocal analysis in $X^{\circ}$ is only at fiber infinity, i.e. at infinite momentum, $\partial \overline{T^{*}} X^{\circ} X=S_{X^{\circ}}^{*} X$.) This reversal corresponds to the need for propagating estimates forward in $\Sigma_{+}$and backward in $\Sigma_{-}$for the operator, with the reverse for the adjoint, corresponding to the 'causal' (Cauchy problem) inverse we need.

Thus, with $x=r^{-1}$ as before, $\chi_{-}$supported in $(0, \infty)$, identically 1 on $\left(x_{1}, \infty\right)$, $x_{1}>0, \tilde{\chi}_{-}$supported in $(0, \infty)$, identically 1 on $\operatorname{supp} \chi_{-}$, and propagating estimates forward along $\mathrm{H}_{p}$ in $\Sigma_{+}$, backwards in $\Sigma_{-}$, one has estimates

$$
\left\|\chi_{-} u\right\|_{\bar{H}^{s}} \leq C\left(\left\|\tilde{\chi}_{-} P(\sigma) u\right\|_{\bar{H}^{s-1}}+\|u\|_{\bar{H}^{-N}}\right)
$$

holding if $s>\frac{1}{2}-\kappa \operatorname{Im} \sigma$, and dually, reversing the direction of propagation,

$$
\left\|\chi_{-} u\right\|_{\dot{H}^{\tilde{s}}} \leq C\left(\left\|\tilde{\chi}_{-} P(\sigma)^{*} u\right\|_{\dot{H}^{\tilde{s}-1}}+\|u\|_{\dot{H}^{-N}}\right) \text {, }
$$

holding if $\tilde{s}<\frac{1}{2}-\kappa \operatorname{Im} \sigma$; for the Fredholm estimate the relevant value of $\tilde{s}$ is $-(s-1)=1-s$, so the inequalities for $s$ and $\tilde{s}$ are equivalent. Here $\bar{H}$ and $\dot{H}$ are, respectively, the Sobolev spaces of extendible and supported distributions, with this property referring to the Cauchy hypersurface; see [24, Section 2.1] in a context closely related to the present one.

As the function spaces are simpler, we first consider the b-, rather than the second microlocal, estimate on the asymptotically Euclidean end. Thus, we let $\tilde{r}$ be a variable order as in the main theorem, with $\beta>0$,

$$
\tilde{r}=\frac{1}{2}-(l+1) \pm \beta \frac{\tau_{\mathrm{b}}}{\left(\tau_{\mathrm{b}}^{2}+\left|\mu_{\mathrm{b}}\right|^{2}\right)^{1 / 2}}, \quad|l+1|<\frac{n-2}{2} .
$$

The estimate on the asymptotically Euclidean end, where $\chi_{+}$can be taken to be $1-\chi_{-}$, while $\tilde{\chi}_{+}$is supported in $\left[0, x_{0}\right)$, identically 1 on supp $\chi_{+}$, is, with $0<K<\beta$,

$$
\left\|\chi_{+} u\right\|_{H_{\mathrm{b}}^{\tilde{r}, l}} \leq C\left(\left\|\tilde{\chi}_{+} P(\sigma) u\right\|_{H_{\mathrm{b}}^{\tilde{r}-1, l+2}}+\|u\|_{H_{\mathrm{b}}^{\tilde{r}-K, l^{\prime}}}\right)
$$


and dually

$$
\|\chi+u\|_{H_{\mathrm{b}}^{1-\tilde{r},-l-2}} \leq C\left(\left\|\tilde{\chi}+P(\sigma)^{*} u\right\|_{H_{\mathrm{b}}^{-\tilde{r},-l}}+\|u\|_{H_{\mathrm{b}}^{\tilde{r}-K, l^{\prime}}}\right)
$$

where $|l+1|<\frac{n-2}{2}$, and $\tilde{r}$ a variable order.

So let $s$ be a variable order satisfying $s>\frac{1}{2}-\kappa \operatorname{Im} \sigma$ near the radial sets at the event horizon, indeed for convenience $s$ constant outside $x<x_{0}$, and $s=\tilde{r}$ near $\partial_{-} X$, we can combine these two (simply use a partition of unity: in the transitional region $P(\sigma)$ is elliptic, so the order is irrelevant) to obtain, with $0<K<\beta$,

$$
\|u\|_{\bar{H}_{\mathrm{b}}^{s, l}} \leq C\left(\|P(\sigma) u\|_{\bar{H}_{\mathrm{b}}^{s-1, l+2}}+\|u\|_{\bar{H}_{\mathrm{b}}^{s-K, l^{\prime}}}\right),
$$

and dually

$$
\|u\|_{\dot{H}_{\mathrm{b}}^{1-s,-l-2}} \leq C\left(\left\|P(\sigma)^{*} u\right\|_{\dot{H}_{\mathrm{b}}^{-s,-l}}+\|u\|_{\dot{H}_{\mathrm{b}}^{s-K, l^{\prime}}}\right) .
$$

We can then argue exactly as in Section 4 in the proof of Proposition 4.4 to remove the compact error term. We state the result both for $P(\sigma)$ and for its adjoint $P(\sigma)^{*}$ since now the dual spaces are not of the same kind due to the artificial boundary, as extendible and supported distributions are interchanged in dualization:

Theorem 6.1. Suppose $P(0): \bar{H}_{\mathrm{b}}^{s, l} \rightarrow \bar{H}_{\mathrm{b}}^{s-2, l+2}$ has trivial nullspace. Then there exist $\sigma_{0}>0, C>0$ such that for $|\sigma|<\sigma_{0}$,

$$
\|u\|_{\bar{H}_{\mathrm{b}}^{s, l}} \leq C\|P(\sigma) u\|_{\bar{H}_{\mathrm{b}}^{s-1, l+2}} .
$$

An analogous statement holds for $P(\sigma)^{*}$ on the dual spaces, provided $P(0)$ : $\dot{H}_{\mathrm{b}}^{1-s,-l-2} \rightarrow \dot{H}_{\mathrm{b}}^{-s,-l}$ has trivial nullspace.

As a consequence, if both $P(0)$ and $P(0)^{*}$ have trivial nullspace on the respective spaces, then there is $\sigma_{0}>0$ such that for $|\sigma|<\sigma_{0}$

$$
P(\sigma):\left\{u \in \bar{H}_{\mathrm{b}}^{s, l}: P(\sigma) u \in \bar{H}_{\mathrm{b}}^{s-1, l+2}\right\} \rightarrow \bar{H}_{\mathrm{b}}^{s-1, l+2}
$$

is invertible, with the inverse possessing uniform bounds.

Finally the second microlocal version is in some sense simpler, in that the order $s$ can be taken to be constant. Namely, with $s>\frac{1}{2}-\kappa \operatorname{Im} \sigma$, and with

$$
r=-\frac{1}{2} \pm \beta \frac{\tau_{\mathrm{b}}}{\left(\tau_{\mathrm{b}}^{2}+\left|\mu_{\mathrm{b}}\right|^{2}\right)^{1 / 2}}=-\frac{1}{2} \pm \beta \frac{\tau}{\left(\tau^{2}+|\mu|^{2}\right)^{1 / 2}},
$$

by Proposition 5.5 one has, for $u \in H_{\mathrm{sc}, \mathrm{b}}^{s^{\prime}, r-K, l^{\prime}}, 0<K<\beta, s^{\prime}, r^{\prime}, l^{\prime}$ arbitrary

$$
\|\chi+u\|_{H_{\mathrm{sc}, \mathrm{b}}^{s, r, l}} \leq C\left(\|\tilde{\chi}+P(\sigma) u\|_{H_{\mathrm{sc}, \mathrm{b}}^{s-1, r+1, l+2}}+\|u\|_{H_{\mathrm{sc}, \mathrm{b}}^{s^{\prime}, r^{\prime}, l^{\prime}}}\right)
$$

and dually

$$
\|\chi+u\|_{H_{\mathrm{sc}, \mathrm{b}}^{1-s,-1-r,-l-2}} \leq C\left(\left\|\tilde{\chi}_{+} P(\sigma)^{*} u\right\|_{H_{\mathrm{sc}, \mathrm{b}}^{-s,-r,-l}}+\|u\|_{H_{\mathrm{sc}, \mathrm{b}}^{s^{\prime}, r^{\prime}, l^{\prime}}}\right)
$$

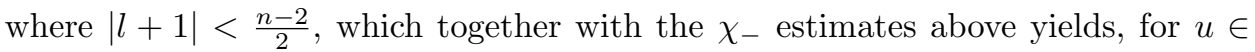
$\bar{H}_{\mathrm{sc}, \mathrm{b}}^{s^{\prime}, r-K, l^{\prime}}, 0<K<\beta$, with $P(\sigma) u \in \bar{H}_{\mathrm{sc}, \mathrm{b}}^{s-1, r+1, l+2}$

$$
\|u\|_{\bar{H}_{\mathrm{sc}, \mathrm{b}}^{s, r, l}} \leq C\left(\|P(\sigma) u\|_{\bar{H}_{\mathrm{sc}, \mathrm{b}}^{s-1, r+1, l+2}}+\|u\|_{\bar{H}_{\mathrm{sc}, \mathrm{b}}^{-N,-N^{\prime}, l^{\prime}}}\right),
$$

and dually (under analogous conditions)

$$
\|u\|_{\dot{H}_{\mathrm{sc}, \mathrm{b}}^{1-s,-1-r,-l-2}} \leq C\left(\left\|P(\sigma)^{*} u\right\|_{\dot{H}_{\mathrm{sc}, \mathrm{b}}^{-s,-r,-l}}+\|u\|_{\dot{H}_{\mathrm{sc}, \mathrm{b}}^{-N,-N^{\prime}, l^{\prime}}}\right) .
$$

Again, arguing exactly as in the proof of Proposition 4.4 we deduce: 
Theorem 6.2. Suppose $P(0): \bar{H}_{\mathrm{sc}, \mathrm{b}}^{s, r, l} \rightarrow \bar{H}_{\mathrm{sc}, \mathrm{b}}^{s-2, r, l+2}$ has trivial nullspace. Then there exist $\sigma_{0}>0, C>0$ such that for $|\sigma|<\sigma_{0}$,

$$
\|u\|_{\bar{H}_{\mathrm{sc}, \mathrm{b}}^{s, r, l}} \leq C\|P(\sigma) u\|_{\bar{H}_{\mathrm{sc}, \mathrm{b}}^{s-1, r+1, l+2}} .
$$

An analogous statement holds for $P(\sigma)^{*}$ on the dual spaces, provided $P(0)^{*}$ : $\dot{H}_{\mathrm{sc}, \mathrm{b}}^{1-s,-r-1,-l-2} \rightarrow \dot{H}_{\mathrm{sc}, \mathrm{b}}^{-s,-r,-l}$ has trivial nullspace.

As a consequence, if both $P(0)$ and $P(0)^{*}$ have trivial nullspace on the respective spaces, then there is $\sigma_{0}>0$ such that for $|\sigma|<\sigma_{0}$

$$
P(\sigma):\left\{u \in \bar{H}_{\mathrm{sc}, \mathrm{b}}^{s, r, l}: P(\sigma) u \in \bar{H}_{\mathrm{sc}, \mathrm{b}}^{s-1, r+1, l+2}\right\} \rightarrow \bar{H}_{\mathrm{sc}, \mathrm{b}}^{s-1, r+1, l+2}
$$

is invertible.

Remark 6.3. As noted in the elliptic setting in Remark 1.6 if $P_{b}(\sigma)$ is a family of operators of the form discussed above for $P(\sigma)$ continuously depending on a parameter $b \in \mathbb{R}^{k}$, and $P_{0}(0)$ is invertible, then there is $\epsilon>0$ such that the same holds for $P_{b}(0)$ for $|b|<\epsilon$, i.e. the hypothesis of the theorem is perturbation stable. In this setting this is an immediate consequence of the stability discussion of [40, Section 2.7] for non-elliptic Fredholm problems.

In particular, the Kerr family is a perturbation of the Schwarzschild family in this sense. Thus, the invertibility of $P_{0}(0)$ for a Schwarzschild metric of a given black hole mass $m>0$ implies the invertibility of $P_{b}(0), b=\left(m^{\prime}, a^{\prime}\right)$, for Kerr metrics of black hole mass $m^{\prime}$ close to $m$ and angular momentum $a^{\prime}$ close to 0 . If $P_{b}(\sigma)$ is the $-t_{*}$-Fourier transformed wave operator on scalar functions, $P_{0}(0)$ is well-known to have trivial nullspace (scalar 'mode stability' of Schwarzschild), going back to Regge and Wheeler [31, and the the same holds for the adjoint. The simplest way to see this is that by a regularization (in terms of decay) argument one shows that for functions in the relevant nullspace, $0=\left\langle P_{0}(0) u, u\right\rangle_{r>r_{0}}=\|d u\|_{r>r_{0}}^{2}$ ); here $r_{0}$ is the event horizon, and there are no boundary terms at the event horizon since the operator is characteristic there. (Notice that $d u$ is in $L^{2}$ automatically since $d$ is a scattering differential operator, so in terms of decay as a b-operator, it is order -1 , and the relevant nullspace is in $H_{\mathrm{b}}^{\infty,-\frac{1}{2}+\epsilon}$ for all $\epsilon>0$.) A different way of proceeding, using spherical symmetry, is that the problem reduces to a onedimensional Schrödinger operator on $\left(r_{0}, \infty\right)$, with a non-negative potential that decays inverse quadratically as $r \rightarrow \infty$ (and smooth at the event horizon), and the relevant nullspace is on functions that are smooth at $r_{0}$ and are in the b-Sobolev spaces discussed in this paper at the other end. Thus, by the perturbation stability corresponding results also hold for slowly rotating Kerr black holes. In particular, in this slowly rotating case it is not necessary to use Whiting's transformation [47] and its extension by Shlapentokh-Rothman [34].

\section{Bound STATES AND HALF-BOUnd STATES}

Finally we consider the case of $P(0)$ having a non-trivial nullspace, at first in the elliptic, in the scattering sense, setting. Before proceeding, we mention the references [26] and [12, Section 3.3] for the precise description of the resolvent near zero energy in potential scattering for sufficiently rapidly decaying, resp. spatially compactly supported, perturbations of the Euclidean Laplacian. 
What happens in this setting is highly dimension dependent for the following reason. For the Fredholm theory, we considered $P(\sigma)$ as a map

$$
\left\{u \in H_{\mathrm{b}}^{\tilde{r}, l}: P(\sigma) u \in H_{\mathrm{b}}^{\tilde{r}-1, l+2}\right\}=\mathcal{X}_{\sigma} \rightarrow H_{\mathrm{b}}^{\tilde{r}-1, l+2},
$$

and the domain here depends on $\sigma$ (though the estimates employed utilize standard variable order Sobolev spaces). Now, in general, in standard meromorphic Fredholm theory, or indeed simply Fredholm theory depending continuously on a parameter, one would decompose the domain as the direct sum of the nullspace of $P(0)$ and a complementary space, and analogously the target space would be decomposed into the range of $P(0)$ and a complementary space, and consider $P(\sigma)$ as a 'block matrix' with respect to this decomposition. Here the choice of the complementary space is flexible, but the nullspace and the range play a key role. Under our assumptions, if $u \in \operatorname{Ker} P(0)$, then for $\sigma \neq 0, u \in \mathcal{X}_{\sigma}$ if and only if $P(\sigma) u \in H_{\mathrm{b}}^{\tilde{r}-1, l+2}$, which is the case if and only if $\sigma^{2} u \in H_{\mathrm{b}}^{\tilde{r}-1, l+2}$, i.e. $u \in H_{\mathrm{b}}^{\tilde{r}-1, l+2}$. Here the differential order is not an issue, since elements of the nullspace are in $H_{\mathrm{b}}^{\infty, l^{\prime}}$ for all $l^{\prime}<-1+\frac{n-2}{2}$, but decay is. Since we need $l>-1-\frac{n-2}{2}$, the statement $u \in \mathcal{X}_{\sigma}$ amounts to $1-\frac{n-2}{2}<-1+\frac{n-2}{2}$, i.e. $n>4$. Thus, for $n \geq 5$, which is exactly when it is guaranteed that there are no half-bound states, one can proceed rather directly with perturbation theory in a Fredholm setting. Namely, choose a complementary subspace to $\operatorname{Ker} P(0)$ in $H_{\mathrm{b}}^{\tilde{r}, l}$ (e.g. the orthocomplement), and a complementary subspace to $\operatorname{Ran} P(0)$ in $H_{\mathrm{b}}^{\tilde{r}-1, l+2}$; consider the complement, resp. $\operatorname{Ker} P(0)$, intersected with $\mathcal{X}_{\sigma}$ as the actual decomposition of the domain, keeping in mind that $\operatorname{Ker} P(0) \subset \mathcal{X}_{\sigma}$ as we already noted (with $l$ chosen appropriately, namely $l<-3+\frac{n-2}{2}$, which is possible for $n \geq 5$ ). Assuming that $P(\sigma)=P(0)-\sigma^{2}$ with $P(0)=P(0)^{*}$ for simplicity, with the decompositions being $\operatorname{Ker} P(0)^{\perp} \oplus \operatorname{Ker} P(0)$ and $\operatorname{Ran} P(0) \oplus \operatorname{Ran} P(0)^{\perp}$, with $\perp$ relative to the $L_{g}^{2}$-inner product to take advantage of the formal self-adjointness, using that $\operatorname{Ran} P(0)^{\perp}=\operatorname{Ker} P(0)\left(\right.$ as $\operatorname{Ker} P(0) \subset H_{\mathrm{b}}^{\infty, l^{\prime}}$, the $L^{2}$-orthocomplement makes sense in $H_{\mathrm{b}}^{\tilde{r}-1, l+2}$ as $l+2+l^{\prime}$ is taken $\geq 0$ for $l^{\prime}+2<1+\frac{n-2}{2}$, arbitrarily close to equality, so even with $l$ barely greater than $-1-\frac{n-2}{2}$, the non-negativity of the sum can be arranged), $P(\sigma)$ becomes the 'block matrix'

$$
\left(\begin{array}{cc}
P(0)-\sigma^{2} & 0 \\
0 & -\sigma^{2}
\end{array}\right) \text {. }
$$

Now the top left entry is invertible for sufficiently small $\sigma$ for the same reasons as in the case $\operatorname{Ker} P(0)$ and $\operatorname{Ker} P(0)^{*}$ were trivial, namely the arguments of Section 4 apply. The actual inverse is thus

$$
\left(\begin{array}{cc}
\left(P(0)-\sigma^{2}\right)^{-1} & 0 \\
0 & -\sigma^{-2}
\end{array}\right)
$$

and thus on the complement of $\operatorname{Ker} P(0)$, the resolvent remains uniformly bounded as $\sigma \rightarrow 0$, while on $\operatorname{Ker} P(0)$ it has the expected $O\left(|\sigma|^{-2}\right)$ asymptotic behavior.

Notice that what we really needed above is not that $n \geq 5$, rather that the nullspace of $P(0)$ is sufficiently decaying, so even if $n \geq 3$ merely, and $\operatorname{Ker} P(0) \subset$ $H_{\mathrm{b}}^{\infty, l+2}$ for some $l>-1-\frac{n-2}{2}$, which is a strengthening of the a priori decay order by $4-n+\epsilon, \epsilon>0$ arbitrarily small, i.e. a strengthening by just a bit more than one order of decay if $n=3$, all the above arguments go through unchanged. Recall that the asymptotic behavior of elements of the nullspace is given by an 
expansion in terms of resonances, which means that the asymptotics are given by powers $\frac{n-2}{2}+\sqrt{\left(\frac{n-2}{2}\right)^{2}+\lambda_{j}}$ of $x$ (plus natural numbers, with possible logarithms), where $\lambda_{j}$ are the eigenvalues of $\Delta_{Y}$, which is to say that these terms lie in

$$
\cap_{\epsilon>0} H_{\mathrm{b}}^{\infty,-1+\sqrt{\left(\frac{n-2}{2}\right)^{2}+\lambda_{j}}-\epsilon} .
$$

For instance, for $n=3$ and $Y$ the standard sphere, $\lambda_{j}=j(j+1), j \geq 0$ integer, and the exponent is $-\frac{1}{2}+j-\epsilon$, which satisfies the desired inequality for $j \geq 2$ (which is stronger than the requirement for being in $L^{2}: j \geq 1$ ). Thus, the only 'non-trivial' part of the nullspace from this perspective is the one with non-vanishing $j=0,1$ terms. We note that this also limits the dimension of the more complicated part of the nullspace to the sum of the dimension of the resonant states (thus of the eigenspaces of the boundary Laplacian) for $j=0,1$.

In order to extend this result, we proceed from a somewhat different perspective which is common in scattering theory. We consider a perturbation $\tilde{P}(\sigma)$ of $P(\sigma)$ such that $\tilde{P}(\sigma)-P(\sigma) \in S^{-2-\delta} \operatorname{Diff}_{\mathrm{b}}^{2}(X)$ and such that $\tilde{P}(0)$ is invertible as a map $H_{\mathrm{b}}^{\tilde{r}, l} \rightarrow H_{\mathrm{b}}^{\tilde{r}, l+2},|l+1|<\frac{n-2}{2}$. Note that such $\tilde{P}(\sigma)$ exists and

$$
V(\sigma)=\tilde{P}(\sigma)-P(\sigma) \in S^{-2-\delta} \operatorname{Diff}_{\mathrm{b}}^{2}(X)
$$

may even be taken to be compactly supported (and even order $-\infty$ ) in $X^{\circ}$, one way of doing this is approximating elements of the nullspaces of $P(0)$ and its adjoint by compactly supported $\mathcal{C}^{\infty}$ functions, another is to add to $P(0)$ a positive 'potential' which is sufficiently large in a sufficiently large compact set. Notice that (even under the weaker assumption), $\mathcal{X}_{\sigma}$ is both the domain for $P(\sigma)$ and for $\tilde{P}(\sigma)$ for all $\sigma$ (as Fredholm operators). Moreover, by our main results, $\tilde{P}(\sigma)$ is invertible for sufficiently small $|\sigma|$ (and $\operatorname{Im} \sigma$ of the correct indefinite sign). Then to work on a domain independent of $\sigma$ we proceed in a standard manner in scattering theory, and consider $P(\sigma) \tilde{P}(\sigma)^{-1}: \mathcal{Y} \rightarrow \mathcal{Y}$; inverting this is equivalent to inverting $P(\sigma)$ in the desired manner.

At first we assume that

$$
V=V(\sigma)
$$

is independent of $\sigma$; later we will relax this assumption. Now,

$$
P(\sigma) \tilde{P}(\sigma)^{-1}=(\tilde{P}(\sigma)-V) \tilde{P}(\sigma)^{-1}=\mathrm{Id}-V \tilde{P}(\sigma)^{-1},
$$

and Id $-V \tilde{P}(\sigma)^{-1}$ is now a bounded, indeed compact, family of operators, continuous in the weak operator topology. Moreover, its nullspace for $\sigma=0$ is the image of that of $P(0)$ under $\tilde{P}(0)$. Thus, elements $v$ of the nullspace satisfy $0=P(0) \tilde{P}(0)^{-1} v=v-V \tilde{P}(0)^{-1} v$, i.e. $v=V \tilde{P}(0)^{-1} v$, so $v$ is in fact compactly supported and smooth if $V$ is arranged to be such. On the other hand, the $L^{2}$ orthocomplement (annihilator) of the range is the nullspace of $P(0)^{*}$ in $\mathcal{Y}^{*}$. We can now consider the decomposition of $\operatorname{Id}-V \tilde{P}(\sigma)^{-1}$ with respect to the orthogonal decomposition of $\mathcal{Y}$ into $\tilde{P}(0) \operatorname{Ker} P(0)$ and its orthocomplement on the domain side (written in the reverse order, compatibly with the preceding discussion), and $\operatorname{Ran} P(0)=\left(\operatorname{Ker} P(0)^{*}\right)^{\perp}$ and $\operatorname{Ker} P(0)^{*}$ on the target space side. With respect to this decomposition, the 00 entry (from the orthocomplement of $\tilde{P}(0) \operatorname{Ker} P(0)$ to $\left.\operatorname{Ran} P(0)=\left(\operatorname{Ker} P(0)^{*}\right)^{\perp}\right)$ of this operator is invertible for $\sigma=0$, and thus for $|\sigma|$ small, with a uniform bound as $\sigma \rightarrow 0$. For all other entries, either the domain is restricted to $\tilde{P}(0) \operatorname{Ker} P(0)$ or the target space is restricted to $\operatorname{Ker} P(0)^{*}$. In the 
former case, using that $P(0) u=0$ means $\tilde{P}(0) u=V u$, this amounts to considering the operator

$$
\begin{aligned}
\left.P(\sigma) \tilde{P}(\sigma)^{-1} \tilde{P}(0)\right|_{\operatorname{Ker} P(0)} & =\tilde{P}(0)-\left.V \tilde{P}(\sigma)^{-1} \tilde{P}(0)\right|_{\operatorname{Ker} P(0)} \\
& =\left.V\left(\operatorname{Id}-\tilde{P}(\sigma)^{-1} \tilde{P}(0)\right)\right|_{\operatorname{Ker} P(0)} \\
& =\left.V\left(\tilde{P}(0)^{-1}-\tilde{P}(\sigma)^{-1}\right) V\right|_{\operatorname{Ker} P(0)}
\end{aligned}
$$

Similarly, taking adjoints, in the latter case, using that $P(0)^{*} \phi=0$ means $\tilde{P}(0)^{*} \phi=$ $V^{*} \phi$, we are considering

$$
\begin{aligned}
\left.\left(\tilde{P}(\sigma)^{-1}\right)^{*} P(\sigma)^{*}\right|_{\operatorname{Ker} P(0)^{*}} & =\operatorname{Id}-\left.\left(\tilde{P}(\sigma)^{-1}\right)^{*} V^{*}\right|_{\operatorname{Ker} P(0)^{*}} \\
& =\left.\left(\left(\tilde{P}(0)^{-1}\right)^{*}-\left(\tilde{P}(\sigma)^{-1}\right)^{*}\right) V^{*}\right|_{\operatorname{Ker} P(0)^{*}}
\end{aligned}
$$

The key point is that one would like to say that $\tilde{P}(0)^{-1}-\tilde{P}(\sigma)^{-1}$ is small, namely the same size as $\tilde{P}(0)-\tilde{P}(\sigma)$. Since

$$
\tilde{P}(0)-\tilde{P}(\sigma)-\sigma^{2}
$$

is a smooth $\sigma$ dependent family of operators in $S^{-2-\delta} \operatorname{Diff}_{\mathrm{b}}^{2}(X)$ vanishing at $\sigma=0$ by assumption, it is convenient to assume that $\tilde{P}(\sigma)$ is chosen so that

$$
\tilde{P}(0)-\tilde{P}(\sigma)=\sigma^{2},
$$

which is thus of $O\left(|\sigma|^{2}\right)$. (Together with the assumption that $V$ is independent of $\sigma$, this means that we are making an assumption on the $\sigma$ dependence of $P(\sigma)$; as already indicated, we will remove this assumption on $V$ in due course, while keeping the assumption $(7.3)$ on $\tilde{P}(\sigma)$.) However, the expectation for $\tilde{P}(0)^{-1}-\tilde{P}(\sigma)^{-1}$ is not always true for domain reasons. Namely, the 'resolvent identity'

$$
\begin{aligned}
\tilde{P}(0)^{-1}-\tilde{P}(\sigma)^{-1} & =\tilde{P}(0)^{-1}(\tilde{P}(\sigma)-\tilde{P}(0)) \tilde{P}(\sigma)^{-1} \\
& =-\sigma^{2} \tilde{P}(0)^{-1} \tilde{P}(\sigma)^{-1}=-\sigma^{2} \tilde{P}(\sigma)^{-1} \tilde{P}(0)^{-1}
\end{aligned}
$$

holds for $\sigma \neq 0$, with the composition justified e.g. as $\tilde{P}(\sigma)$ is elliptic near the 0 -section of the scattering cotangent bundle, but this is of course not a uniform statement as $\sigma \rightarrow 0$, hence this expression fails to be $O\left(|\sigma|^{2}\right)$ in general. Indeed, the issue with the composition is that acting on $H_{\mathrm{b}}^{\tilde{r}-1, l+2}, \tilde{P}(\sigma)^{-1}\left(\right.$ resp. $\left.\tilde{P}(0)^{-1}\right)$ produces an element of $H_{\mathrm{b}}^{\tilde{r}, l}$ (uniformly bounded in $\sigma$ ), but as $l<-1+\frac{n-2}{2}$, this is in the domain of $\tilde{P}(0)^{-1}$ (resp. $\tilde{P}(\sigma)^{-1}$ ) only if $-1+\frac{n-2}{2}>1-\frac{n-2}{2}$ (i.e. $n>4$ ), in which case one can indeed make sense of the composition in a uniformly bounded way. Notice that for $n=4$, the failure of the composition making sense is just the lack of an additional $\epsilon$ order decay, while for $n=3$ the lack of an additional $1+\epsilon$ order of decay, where $\epsilon>0$ can be taken arbitrarily small.

However:

Lemma 7.1. For $0 \leq s \leq 2, l, \tilde{r}$ as before, the operator $\sigma^{2} \tilde{P}(\sigma)^{-1}$ is bounded by $C|\sigma|^{s}$ as a map

$$
H_{\mathrm{b}}^{\tilde{r}-1, l+2} \rightarrow H_{\mathrm{b}}^{\tilde{r}-2+s, l+2-s}, 0 \leq s \leq 2 .
$$

Remark 7.2. Here the key part is the decay order as that is what prevents the composition $\tilde{P}(0)^{-1} \tilde{P}(\sigma)^{-1}$ from being uniformly bounded; the point is that we can improve the mapping property (the target weight) by making $s$ smaller, i.e. by giving up on decay as $|\sigma| \rightarrow 0$. Furthermore, since we are interested in this expression either when applied to elements of $V \operatorname{Ker} P(0)$, or paired with elements 
of $V^{*} \operatorname{Ker} P(0)^{*}$, both of which have infinite b-differential regularity, the differential orders never matter for us.

Proof. We have

$$
\sigma^{2} \tilde{P}(\sigma)^{-1}=(\tilde{P}(0)-\tilde{P}(\sigma)) \tilde{P}(\sigma)^{-1}=-\mathrm{Id}+\tilde{P}(0) \tilde{P}(\sigma)^{-1} .
$$

Now, our results for $\tilde{P}(\sigma)$ imply that the left hand side is bounded by $C|\sigma|^{2}$ as a map $H_{\mathrm{b}}^{\tilde{r}-1, l+2} \rightarrow H_{\mathrm{b}}^{\tilde{r}, l}$, while the right hand side is bounded by $C$ as a map $H_{\mathrm{b}}^{\tilde{r}-1, l+2} \rightarrow$ $H_{\mathrm{b}}^{\tilde{r}-2, l+2}$. By interpolation, this proves the lemma since the interpolation spaces between $H_{\mathrm{b}}^{\tilde{r}, l}$ and $H_{\mathrm{b}}^{\tilde{r}-2, l+2}$ are exactly $H_{\mathrm{b}}^{\tilde{r}-2+s, l+2-s}, 0 \leq s \leq 2$. Recall that this can instead be rephrased using a holomorphic family of operators which are an isomorphism between $H_{\mathrm{b}}^{\tilde{r}-2+s, l+2-s}, 0 \leq s \leq 2$, and the base case, $s=0$. Following a suggestion of Hintz, one can achieve this particularly simply by not using complex powers of a single operator rather a family of the form $x^{2 z} L^{z}, L \in \operatorname{Diff}_{\mathrm{b}}^{2}(X)$ is formally self-adjoint, elliptic with normal operator invertible on the reals, e.g. the Laplacian of a Riemannian b-metric (asymptotically cylindrical metric) plus 1, with boundedness of $x^{2 z} L^{z} \sigma^{-2 z} \tilde{P}(\sigma)$ as a map $H_{\mathrm{b}}^{\tilde{r}-1, l+2} \rightarrow H_{\mathrm{b}}^{\tilde{r}-2, l+2}$ with $\operatorname{Re} z=0,1$ clear, and thus interpolation proving it for all $z$, and thus the lemma for all $s$.

This lemma gives, for $n=4$, that $(7.4)$ is bounded by $C|\sigma|^{2-\epsilon}$, while for $n=3$ by $C|\sigma|^{1-\epsilon}, \epsilon>0$ arbitrary, between appropriate spaces. In view of the presence of $V$ or $V^{*}$ in 7.1 and 7.2 , the precise spaces in between which we have the composition bound makes little difference, provided that $V$ maps the full range of $\mathcal{X}_{\sigma} \subset H_{\mathrm{b}}^{\tilde{r}, l}$ to the full range of $\mathcal{Y}=H_{\mathrm{b}}^{\tilde{r}-1, l^{\prime}+2}$ of unrelated decay order $l^{\prime}$ (in the acceptable range of decay orders, so $\left.\left|l^{\prime}+1\right|<\frac{n-2}{2}\right)$, that is provided that $V \in S^{-\alpha} \operatorname{Diff}_{\mathrm{b}}^{2}(X)$ with $\alpha \geq\left(1+\frac{n-2}{2}\right)-\left(-1-\frac{n-2}{2}\right)=n$.

For later use, we note that assuming that we restrict to a subspace of $\operatorname{Ker} P(0)$ or $\operatorname{Ker} P(0)^{*}$ with better decay properties, the statement can be strengthened. For instance, writing

$$
\left.\left(\tilde{P}(0)^{-1}-\tilde{P}(\sigma)^{-1}\right) V\right|_{\operatorname{Ker} P(0)}=-\left.\sigma^{2} \tilde{P}(\sigma)^{-1} \tilde{P}(0)^{-1} V\right|_{\operatorname{Ker} P(0)}=-\left.\sigma^{2} \tilde{P}(\sigma)^{-1}\right|_{\operatorname{Ker} P(0)},
$$

if we restrict to a subspace of $\operatorname{Ker} P(0)$ which lies in $\cap_{\epsilon>0} H_{\mathrm{b}}^{\infty,(n-4) / 2+j-\epsilon}$, then we can reduce the loss of order of vanishing by $j$ (but not to faster than quadratic vanishing), i.e. when $n=3$ and $Y$ is the standard sphere we have an a priori bound of $C|\sigma|^{2-\epsilon}$ for $j=1$, and (consistent with how $j \geq 2$ directly fit into the perturbation theory discussion) a $C|\sigma|^{2}$ bound for $j \geq 2$.

From now on we concentrate on the most interesting case of $n=3$, also because it is more singular than the $n=4$ case. For the sake of definiteness, we will only consider the case when $Y$ is the standard sphere. It is convenient to further refine the block matrix decomposition by decomposing the 11 block into the $j=0$ and the $j \geq 1$ parts, with the precise meaning that the the $j \geq 1$ part lies in $\cap_{\epsilon>0} H_{\mathrm{b}}^{\infty,(n-4) / 2+1-\epsilon}$, while the $j=0$ is complementary to this in $\operatorname{Ker} P(0)$ (or $\left.\operatorname{Ker} P(0)^{*}\right)$. From now on we change the indexing accordingly, the operator will be regarded as a 3-by-3 block matrix, with the previous column, resp. row, 1 will be replaced by 2 columns, resp. rows, denoted by 1 and 2 , with 1 corresponding to $j=0,2$ corresponding to $j \geq 1$.

Our argument so far proves:

Proposition 7.3. Suppose that $V=V(\sigma)$ is independent of $\sigma$ and $n=3$. 
The entries of the block matrix of $P(\sigma) \tilde{P}(\sigma)^{-1}$ in the above sense have bounds

$$
\left(\begin{array}{ccc}
O(1) & O\left(|\sigma|^{1-\epsilon}\right) & O\left(|\sigma|^{2-\epsilon}\right) \\
O\left(|\sigma|^{1-\epsilon}\right) & O\left(|\sigma|^{1-\epsilon}\right) & O\left(|\sigma|^{2-\epsilon}\right) \\
O\left(|\sigma|^{2-\epsilon}\right) & O\left(|\sigma|^{2-\epsilon}\right) & O\left(|\sigma|^{2-\epsilon}\right)
\end{array}\right),
$$

with $\epsilon>0$ arbitrary, and furthermore the 00 entry has a bounded inverse.

We next strengthen this if $\tilde{P}(\sigma)$ is the spectral family of the Euclidean Laplacian:

Proposition 7.4. Suppose that $V=V(\sigma)$ is independent of $\sigma$, and that $\tilde{P}(\sigma)=$ $\Delta_{\mathbb{R}^{n}}-\sigma^{2}, n=3$.

Consider the block matrix of Proposition 7.3. Suppose that the $L^{2}$ pairing between the $j \geq 1$ part of $\operatorname{Ker} P(0)$ and the corresponding part of $\operatorname{Ker} P(0)^{*}$ is non-degenerate, and that the $L^{2}(Y)$ pairing between the leading asymptotic terms of the $j=0$ parts of $\operatorname{Ker} P(0)$ and $\operatorname{Ker} P(0)^{*}$ is also non-degenerate.

Then for $\sigma \neq 0$, the 11, resp. 22 entries are invertible with bounds $C|\sigma|$, resp. $C|\sigma|^{2}$, and with the respected inverses bounded by $C|\sigma|^{-1}$, resp. $C|\sigma|^{-2}$, while the 12 and 21 entries are bounded by $C|\sigma|^{2}$.

Here the lower right 2-by-2-block in fact has entries that are multiples of the indicated power of $\sigma$ up to a term with an extra $O\left(|\sigma|^{1-\epsilon}\right)$ vanishing, and the diagonal entries have inverses that are multiples of the indicated power of $\sigma^{-1}$, again up to a term with an extra $O\left(|\sigma|^{1-\epsilon}\right)$ vanishing.

Note that in particular the non-degeneracy of the pairing of $\operatorname{Ker} P(0)$ and $\operatorname{Ker} P(0)^{*}$ holds if $P(0)=P(0)^{*}$.

Proof. Notice that this proposition is purely a statement involving columns and rows 1 and 2, thus in both the 'input' and the 'output' slots we are restricted to elements of (the image of) $\operatorname{Ker} P(0), \operatorname{resp} \operatorname{Ker} P(0)^{*}$.

The 11, 12, 21 and 22 entries are still higher (but finite) rank in general; we evaluate them on an element $u$ of $\operatorname{Ker} P(0)$ paired with an element $\phi$ of $\operatorname{Ker} P(0)^{*}$. Thus, we need to evaluate

$$
\left\langle\left(\tilde{P}(0)^{-1}-\tilde{P}(\sigma)^{-1}\right) V u, V^{*} \phi\right\rangle .
$$

Now $\tilde{P}(0)^{-1}-\tilde{P}(\sigma)^{-1}$ is a Fourier multiplier by

$$
|\xi|^{-2}-\left(|\xi|^{2}-(\sigma \pm i 0)^{2}\right)^{-1}=-\sigma^{2}|\xi|^{-2}\left(|\xi|^{2}-(\sigma \pm i 0)^{2}\right)^{-1}
$$

and thus 7.6 is, with the hat denoting the Fourier transform,

$$
-(2 \pi)^{-3} \int_{\mathbb{R}^{3}} \sigma^{2}|\xi|^{-2}\left(|\xi|^{2}-(\sigma \pm i 0)^{2}\right)^{-1} \widehat{V u}(\xi) \widehat{V^{*} \phi}(\xi) d \xi
$$

Changing variables to $\eta=\xi /|\sigma|$, we obtain, with $\hat{\sigma}=\frac{\sigma}{|\sigma|}$,

$$
-(2 \pi)^{-3} \sigma^{2}|\sigma|^{-1} \int_{\mathbb{R}^{3}}|\eta|^{-2}\left(|\eta|^{2}-(\hat{\sigma} \pm i 0)^{2}\right)^{-1} \widehat{V u}(|\sigma| \eta) \widehat{V^{*} \phi}(|\sigma| \eta) d \eta .
$$

Now, as $\eta \mapsto|\eta|^{-2}\left(|\eta|^{2}-(\hat{\sigma} \pm i 0)\right)^{-1}$ is $L^{1}$ away from $|\eta|=1$, and near $|\eta|=1$ it can be considered a distribution of compact support (technically one uses a partition of unity to combine these two results), the integral is uniformly bounded as $\sigma \rightarrow 0$, and indeed converges as $\sigma \rightarrow 0$ to

$$
\widehat{V u}(0) \widehat{V^{*} \phi}(0) \int_{\mathbb{R}^{3}}|\eta|^{-2}\left(|\eta|^{2}-(\hat{\sigma} \pm i 0)^{2}\right)^{-1} d \eta .
$$


This new integral can be computed, for the sake of definiteness for the + sign in \pm (the other case is the complex conjugate) by writing it as

$$
\begin{aligned}
& \int_{\mathbb{R}^{3}}|\eta|^{-2}\left(|\eta|^{2}-(\hat{\sigma}+i 0)^{2}\right)^{-1} d \eta=4 \pi \int_{0}^{\infty}\left(\rho^{2}-(\hat{\sigma}+i 0)^{2}\right)^{-1} d \rho \\
& =2 \pi \hat{\sigma}^{-1} \int_{0}^{\infty}\left((\rho-(\hat{\sigma}+i 0))^{-1}-(\rho+(\hat{\sigma}+i 0))^{-1}\right) d \rho \\
& =\left.2 \pi \hat{\sigma}^{-1} \log \frac{\rho-(\hat{\sigma}+i 0)}{\rho+(\hat{\sigma}+i 0)}\right|_{0} ^{\infty}=-2 \pi(-\pi i) \hat{\sigma}^{-1}=2 \pi^{2} i \hat{\sigma}^{-1},
\end{aligned}
$$

which is non-zero. Now, $\widehat{V u}(0)$ is a non-vanishing multiple of the leading asymptotic coefficient, namely that of $x=r^{-1}$, of $\tilde{P}(0)^{-1} V u$ since the latter is the inverse Fourier transform of $|\xi|^{-2} \widehat{V u}$ (so the multiple is actually $4 \pi$ in view of the inverse Fourier transform of $\left.|\xi|^{-2}\right)$, but as $V u=\tilde{P}(0) u$, this is exactly $\tilde{P}(0)^{-1} V u=u$. Thus, the 11 entry is actually a constant $\left(\frac{1}{4 \pi i}(4 \pi)^{2}\right)$ times $\sigma$ times the pairing between the leading (constant!) coefficient of the expansion of $\operatorname{Ker} P(0)$ and $\operatorname{Ker} P(0)^{*}$. Thus, the 11 entry is invertible for $\sigma \neq 0$, and the inverse is bounded by $C|\sigma|^{-1}$. Correspondingly, in the absence of the $j \geq 1$ parts (column and row 2 of the matrix) $P(\sigma) \tilde{P}(\sigma)^{-1}$, and thus $P(\sigma)$, is invertible for $\sigma \neq 0$, with a bound $C|\sigma|^{-1}$, and in the block decomposition the inverse is

$$
\left(\begin{array}{cc}
O(1) & O\left(|\sigma|^{-\epsilon}\right) \\
O\left(|\sigma|^{-\epsilon}\right) & O\left(|\sigma|^{-1}\right)
\end{array}\right)
$$

with the leading term of the 11 entry being $4 \pi i \sigma^{-1}$ if $u$ is normalized to have leading term $\frac{x}{4 \pi}$.

This computation also shows that if either $u$ or $\phi$ have vanishing leading term, then indeed the pairing in $O\left(|\sigma|^{2}\right)$. Hence it remains to compute the 22 block. For this we note that

$$
\begin{aligned}
& \left\langle\left(\tilde{P}(0)^{-1}-\tilde{P}(\sigma)^{-1}\right) V u, V^{*} \phi\right\rangle \\
& =-\sigma^{2}\left\langle\tilde{P}(\sigma)^{-1} V u,\left(\tilde{P}(0)^{-1}\right)^{*} V^{*} \phi\right\rangle \\
& =-\sigma^{2}\left\langle\tilde{P}(0)^{-1} V u, \phi\right\rangle-\sigma^{2}\left\langle\left(\tilde{P}(\sigma)^{-1}-\tilde{P}(0)\right)^{-1} V u, \phi\right\rangle \\
& =-\sigma^{2}\langle u, \phi\rangle+\sigma^{2}\left\langle\sigma^{2} \tilde{P}(\sigma)^{-1} \tilde{P}(0)^{-1} V u, \phi\right\rangle \\
& =-\sigma^{2}\langle u, \phi\rangle+\sigma^{2}\left\langle\sigma^{2} \tilde{P}(\sigma)^{-1} u, \phi\right\rangle
\end{aligned}
$$

Now, the preceding discussion shows that the second term is $O\left(|\sigma|^{3-\epsilon}\right)$, so the 22 entry of the block matrix is simply $-\sigma^{2}$ times the pairing between $\operatorname{Ker} P(0)$ and $\operatorname{Ker} P(0)^{*}$, modulo $O\left(|\sigma|^{2}\right)$, so as long as this pairing is non-degenerate, the block is invertible with an $O\left(|\sigma|^{-2}\right)$ inverse, completing the proof.

From this proposition a standard argument, using (block-)Gaussian elimination, immediately shows:

Theorem 7.5. Under the assumptions of Proposition 7.4, the whole block matrix of $P(\sigma) \tilde{P}(\sigma)^{-1}$ is invertible for $\sigma \neq 0$ with inverse having block matrix with bounds

$$
\left(\begin{array}{ccc}
O(1) & O\left(|\sigma|^{-\epsilon}\right) & O\left(|\sigma|^{-\epsilon}\right) \\
O\left(|\sigma|^{-\epsilon}\right) & O\left(|\sigma|^{-1}\right) & O\left(|\sigma|^{-1}\right) \\
O\left(|\sigma|^{-\epsilon}\right) & O\left(|\sigma|^{-1}\right) & O\left(|\sigma|^{-2}\right)
\end{array}\right) .
$$

Consequently, $P(\sigma)^{-1}$ has the same structure. 
Here the lower right 2-by-2-block in fact has entries that are smooth multiples of the indicated power of $\sigma^{-1}$ up to a term with an extra $O\left(|\sigma|^{1-\epsilon}\right)$ vanishing.

Note that this theorem can be applied if $P(\sigma)=P(0)-\sigma^{2}$ is a spectral family on $X=\overline{\mathbb{R}^{n}}$, with $Y$ the standard Riemannian sphere, with no additional assumptions on $P(\sigma)$. A key consequence of this for applications is that when applied to elements of the complement of $\operatorname{Ker} P(0), P(\sigma)^{-1}$ is bounded by $O\left(|\sigma|^{-\epsilon}\right)$, and the same holds when $P(\sigma)^{-1}$ is applied to any element of the domain, provided the result is projected to $\operatorname{Ran} P(0)$.

Finally we consider the case of $P(\sigma)$ being a Kerr-type operator, i.e. $X$ has two boundary hypersurfaces $\partial_{+} X=Y$ and $\partial_{-} X$ as in the previous section, with the former being the Euclidean end, and with $V(\sigma)$ not assumed to be independent of $\sigma$. Notice that with this arrangement one may keep $\tilde{P}(\sigma)-\tilde{P}(0)=-\sigma^{2}$, even if $P(\sigma)$ equals the Kerr 'spectral family' near the Euclidean end $\partial_{+} X$ and one wants $\tilde{P}(\sigma)$ to be the spectral family of the Euclidean Laplacian near $\partial_{+} X$.

We prove:

Theorem 7.6. Suppose that $\tilde{P}(\sigma)$ equals $\Delta_{\mathbb{R}^{n}}-\sigma^{2}, n=3$, near $\partial_{+} X$.

Consider the block matrix of Proposition 7.3. Suppose that the $L^{2}$ pairing between the $j \geq 1$ part of $\operatorname{Ker} P(0)$ and the corresponding part of $\operatorname{Ker} P(0)^{*}$ is non-degenerate, and that the $L^{2}(Y)$ pairing between the leading $\partial_{+} X$-asymptotic terms of the $j=0$ parts of $\operatorname{Ker} P(0)$ and $\operatorname{Ker} P(0)^{*}$ is also non-degenerate.

Suppose also that $V(\sigma)-V(0)$ annihilates $\operatorname{Ker} P(0)$ and of $\operatorname{Ker} P(0)^{*}$.

Then the whole block matrix of $P(\sigma) \tilde{P}(\sigma)^{-1}$ is invertible for $\sigma \neq 0$ with inverse having block matrix with bounds

$$
\left(\begin{array}{ccc}
O(1) & O\left(|\sigma|^{-\epsilon}\right) & O\left(|\sigma|^{-\epsilon}\right) \\
O\left(|\sigma|^{-\epsilon}\right) & O\left(|\sigma|^{-1}\right) & O\left(|\sigma|^{-1}\right) \\
O\left(|\sigma|^{-\epsilon}\right) & O\left(|\sigma|^{-1}\right) & O\left(|\sigma|^{-2}\right)
\end{array}\right) .
$$

Consequently, $P(\sigma)^{-1}$ has the same structure.

Here the lower right 2-by-2-block in fact has entries that are smooth multiples of the indicated power of $\sigma^{-1}$ up to a term with an extra $O\left(|\sigma|^{1-\epsilon}\right)$ vanishing.

Remark 7.7. As follows from the proof below, it suffices to make the assumption on $V(\sigma)-V(0)$ for the $j \geq 1$ part of $\operatorname{Ker} P(0)$ and of $\operatorname{Ker} P(0)^{*}$, provided $V(\sigma)-V(0)$ has sufficiently small coefficients.

Remark 7.8. Notice that if, within our framework, one perturbs an operator for which the non-degeneracy of the pairings is known, the same non-degeneracy will hold for sufficiently small perturbations.

Remark 7.9. If one has a family of operators $P_{b}(\sigma)$ as in Remark 1.6. cf. Remark6.3, and performs the block matrix decomposition for $P_{0}(0)$, then the 00 block remains invertible for $P_{b}(0),|b|$ sufficiently small, by perturbation stability. On the other hand, in general, $\operatorname{Ker} P_{b}(0)$ and $\operatorname{Ker} P_{b}(0)^{*}$ may become lower dimensional. However, if one a priori knows that there are subspaces of $\operatorname{Ker} P_{b}(0)$ and $\operatorname{Ker} P_{b}(0)^{*}$ which vary continuously with $b$, and for $b=0$ are $\operatorname{Ker} P_{0}(0)$ and $\operatorname{Ker} P_{0}(0)^{*}$, then these are necessarily all of $\operatorname{Ker} P_{b}(0)$ and $\operatorname{Ker} P_{b}(0)^{*}$ since the dimension of the latter spaces (for $b \neq 0,|b|$ small) is bounded by that of that of the former spaces (by block-Gaussian elimination). In addition, in this case, the pairings in Theorem 7.6. themselves being continuous in $b$, are non-degenerate as well for $b \neq 0$, i.e. the theorem is equally applicable then. 
Proof. We start the proof with a simpler setting, when $X=\overline{\mathbb{R}^{n}}$ for now with $Y$ the standard sphere, but we allow $V$ to depend on $\sigma$, so $V(\sigma)=\tilde{P}(\sigma)-P(\sigma)$. (So this in particular already incorporates the behavior of the Kerr spectral family near $\partial_{+} X$.) In this case $\tilde{P}(0)^{-1} V(0)$ is the identity on $\operatorname{Ker} P(0)$. Moreover,

$$
\begin{aligned}
& \left.P(\sigma) \tilde{P}(\sigma)^{-1} \tilde{P}(0)\right|_{\operatorname{Ker} P(0)} \\
& =\tilde{P}(0)-V(0) \tilde{P}(\sigma)^{-1} \tilde{P}(0)-\left.(V(\sigma)-V(0)) \tilde{P}(\sigma)^{-1} \tilde{P}(0)\right|_{\operatorname{Ker} P(0)} \\
& =V(0)\left(\operatorname{Id}-\tilde{P}(\sigma)^{-1} \tilde{P}(0)\right)-\left.(V(\sigma)-V(0)) \tilde{P}(\sigma)^{-1} \tilde{P}(0)\right|_{\operatorname{Ker} P(0)} \\
& =V(0)\left(\tilde{P}(0)^{-1}-\tilde{P}(\sigma)^{-1}\right) V(0)-\left.(V(\sigma)-V(0)) \tilde{P}(\sigma)^{-1} V(0)\right|_{\operatorname{Ker} P(0)} .
\end{aligned}
$$

Now the first term behaves exactly as before, when $V$ was assumed to be independent of $\sigma$, while for the second term we can write

$$
\begin{aligned}
& \left.(V(\sigma)-V(0)) \tilde{P}(\sigma)^{-1} V(0)\right|_{\operatorname{Ker} P(0)} \\
& =(V(\sigma)-V(0))+\left.(V(\sigma)-V(0))\left(\tilde{P}(\sigma)^{-1}-\tilde{P}(0)^{-1}\right) V(0)\right|_{\operatorname{Ker} P(0)} .
\end{aligned}
$$

The second term of this expression can be analyzed as before, with the prefactor $V(\sigma)-V(0)$ giving an extra $\sigma$ vanishing, thus it contributes $O\left(|\sigma|^{2-\epsilon}\right)$ in all cases, indeed $O\left(|\sigma|^{3-\epsilon}\right)$ in the $j \geq 1$ input case (i.e. column 2). On the other hand, in general, the first term has a non-trivial $O(|\sigma|)$ contribution.

Similarly, taking adjoints, in the latter case, using that $P(0)^{*} \phi=0$ means $\tilde{P}(0)^{*} \phi=V(0)^{*} \phi$, we are considering

$$
\begin{aligned}
& \left.\left(\tilde{P}(\sigma)^{-1}\right)^{*} P(\sigma)^{*}\right|_{\operatorname{Ker} P(0)^{*}}=\operatorname{Id}-\left.\left(\tilde{P}(\sigma)^{-1}\right)^{*} V(\sigma)^{*}\right|_{\operatorname{Ker} P(0)^{*}} \\
& =\left(\left(\tilde{P}(0)^{-1}\right)^{*}-\left(\tilde{P}(\sigma)^{-1}\right)^{*}\right) V(0)^{*}-\left.\left(\tilde{P}(\sigma)^{-1}\right)^{*}(V(\sigma)-V(0))^{*}\right|_{\operatorname{Ker} P(0)^{*}}
\end{aligned}
$$

Again, the first term is as before, while for the second

$$
\begin{aligned}
& \left.\left(\tilde{P}(\sigma)^{-1}\right)^{*}(V(\sigma)-V(0))^{*}\right|_{\operatorname{Ker} P(0)^{*}} \\
& =\left(\tilde{P}(0)^{-1}\right)^{*}(V(\sigma)-V(0))^{*}+\left.\left(\tilde{P}(\sigma)^{-1}-\tilde{P}(0)^{-1}\right)^{*}(V(\sigma)-V(0))^{*}\right|_{\operatorname{Ker} P(0)^{*}},
\end{aligned}
$$

and one again has an $O\left(|\sigma|^{2-\epsilon}\right)$ estimate for the new second term, while the first is $O(|\sigma|)$.

Now, $O\left(|\sigma|^{2-\epsilon}\right)$ entries were negligible in our preceding discussion except for the 22 entry, where $o\left(|\sigma|^{2}\right)$ entries are negligible, thus $O\left(|\sigma|^{3-\epsilon}\right)$ is in particular always negligible. Thus, the second term of $\sqrt{7.10}$ and $(7.12)$ are both negligible for our purposes, and the only potential issue is the behavior of the first term in both of these, since it is only $O(|\sigma|)$. Such terms were negligible in our preceding discussion except for the $02,20,11,12,21$ and 22 entries, and in fact small $O(|\sigma|)$ entries are negligible even there except for the 12, 21 (where small $O\left(|\sigma|^{3 / 2}\right.$ ) works) and 22 entries (where we need small $O\left(|\sigma|^{2}\right)$ ), by the Gaussian elimination argument (though the inverse will have larger entries in the 02, 12, 20 and 21 slots).

So if we assume that $V(\sigma)-V(0)$ annihilates the $j \geq 1$ part of $\operatorname{Ker} P(0)$ and its adjoint of $\operatorname{Ker} P(0)^{*}$, and is small on the $j=0$ part (the 11 slot), we have exactly the same result as if $V$ is independent of $\sigma$, namely the analogue of Proposition 7.4 holds. This proves Theorem 7.6 under the stronger assumption on $X$.

It remains to deal with the Cauchy hypersurface, $\partial_{-} X$ and to ensure that all of our arguments go through in this case as well. All of the discussion concerning $V(\sigma)$ above remain valid, i.e. the new terms with $V(\sigma)-V(0)$ can be handled as above. Moreover, the computation leading to 7.8 remains valid even in this case 
as well. Thus, in order to show the analogue of Proposition 7.4 it remains to show that even in this more general setting the contribution of the first term of the right hand sides of 7.9 and (7.11) to the 12 and 21 blocks are $O\left(|\sigma|^{2}\right)$ and compute the 11 block. For this we introduce an auxiliary differential operator $\tilde{P}_{0}(\sigma)$ that equals $\tilde{P}(\sigma)$ near the Euclidean end, $\partial_{+} X$, so that if $\chi \in \mathcal{C}^{\infty}(X ; \mathbb{R})$ identically 1 near $\partial_{+} X$, supported away from $\partial_{-} X$, then $\chi P(\sigma)=\chi \tilde{P}(\sigma)$. Here we can consider $\tilde{P}_{0}(\sigma)$ as an operator on a different manifold; concretely, in the case of interest, of $Y$ being the standard sphere, we replace $X$ by $\tilde{X}=\overline{\mathbb{R}^{n}}$, and assume that $\tilde{P}_{0}(\sigma)$ is an operator on this space, and indeed that it is the spectral family of the Euclidean Laplacian.

Then

$$
\tilde{P}_{0}(\sigma) \chi \tilde{P}(\sigma)^{-1} V(\sigma) u=\left(\chi+\left[\tilde{P}_{0}(\sigma), \chi\right] \tilde{P}(\sigma)^{-1}\right) V(\sigma) u
$$

in $\mathcal{Y}$, thus

$$
\chi \tilde{P}(\sigma)^{-1} V(\sigma) u=\tilde{P}_{0}(\sigma)^{-1}\left(\chi+\left[\tilde{P}_{0}(\sigma), \chi\right] \tilde{P}(\sigma)^{-1}\right) V(\sigma) u,
$$

and similarly for $\chi \tilde{P}(0)^{-1} V(\sigma) u$. Notice that

$$
\tilde{P}(\sigma)^{-1} V(\sigma) u, \chi \tilde{P}(\sigma)^{-1} V(\sigma) u, \tilde{P}_{0}(\sigma)^{-1}\left(\chi+\left[\tilde{P}_{0}(\sigma), \chi\right] \tilde{P}(\sigma)^{-1}\right) V(\sigma) u
$$

thus have the same asymptotic behavior near $\partial_{+} X$, since they are actually equal there.

Now,

$$
\begin{aligned}
& \left\langle\left(\tilde{P}(0)^{-1}-\tilde{P}(\sigma)^{-1}\right) V(\sigma) u, V(\sigma)^{*} \phi\right\rangle \\
& =-\sigma^{2}\left\langle\tilde{P}(\sigma)^{-1} V(\sigma) u,\left(\tilde{P}(0)^{-1}\right)^{*} V(\sigma)^{*} \phi\right\rangle \\
& =-\sigma^{2}\left\langle\chi \tilde{P}(\sigma)^{-1} V(\sigma) u, \chi\left(\tilde{P}(0)^{-1}\right)^{*} V(\sigma)^{*} \phi\right\rangle \\
& \quad \quad-\sigma^{2}\left\langle\left(1-\chi^{2}\right) \tilde{P}(\sigma)^{-1} V(\sigma) u,\left(\tilde{P}(0)^{-1}\right)^{*} V(\sigma)^{*} \phi\right\rangle
\end{aligned}
$$

Due to $1-\chi^{2}$ vanishing near $\partial_{+} X$, the composition $\tilde{P}(0)^{-1}\left(1-\chi^{2}\right) \tilde{P}(\sigma)$ remains uniformly bounded as $|\sigma| \rightarrow 0$, so the second term is $O\left(|\sigma|^{2}\right)$, so it remains to analyze the first term, which is

$$
-\sigma^{2}\left\langle\tilde{P}_{0}(\sigma)^{-1} f,\left(\tilde{P}_{0}(0)^{-1}\right)^{*} \psi\right\rangle
$$

with $f=\left(\chi+\left[\tilde{P}_{0}(\sigma), \chi\right] \tilde{P}(\sigma)^{-1}\right) V(\sigma) u$, etc. But now $\tilde{P}_{0}(\sigma)$ is the spectral family of the Euclidean Laplacian, so our computation from the proof of Proposition 7.4 is applicable, replacing $V(\sigma) u$ by $f$, and $V(\sigma)^{*} \phi$ by $\psi$. Now, as remarked after (7.7), the 11 entry is the pairing between the leading asymptotic coefficients of $\tilde{P}_{0}(\sigma)^{-1} f$ and $\left(\tilde{P}_{0}(0)^{-1}\right)^{*} \psi$, thus, in view of 77.13$)$, of $\tilde{P}(\sigma)^{-1} V(\sigma) u$ and $\left(\tilde{P}(0)^{-1}\right)^{*} V(\sigma)^{*} \phi$, i.e. in terms of the asymptotics, it is given by exactly the same expression as beforehand! Similarly, the 12 and 21 entries are $O\left(|\sigma|^{2}\right)$ since this leading term vanishes. In summary, the structure of our block matrix is the same even in the presence of Cauchy hypersurfaces $\partial_{-} X$, provided the pairing between the leading $\partial_{+} X$ asymptotic terms of the $j=0$ resonant states and dual states is non-degenerate, and provided that the $L^{2}$-pairing between the $j \geq 1$ resonant states and dual states is non-degenerate, completing the proof.

Remark 7.10. A simple extension of the proof of this theorem shows that if $\operatorname{Ker} P(0)$ has a non-trivial $j=0$ component, but $\operatorname{Ker} P(0)^{*}$ does not, choosing a nondegenerate dual decomposition of $\operatorname{Ker} P(0)^{*}$ (assuming the existence of this), the 
structure of $P(\sigma) \tilde{P}(\sigma)^{-1}$ is

$$
\left(\begin{array}{ccc}
O(1) & O\left(|\sigma|^{1-\epsilon}\right) & O\left(|\sigma|^{2-\epsilon}\right) \\
O\left(|\sigma|^{2-\epsilon}\right) & O\left(|\sigma|^{2}\right) & O\left(|\sigma|^{2}\right) \\
O\left(|\sigma|^{2-\epsilon}\right) & O\left(|\sigma|^{2}\right) & O\left(|\sigma|^{2}\right)
\end{array}\right),
$$

where non-degeneracy means that the 2-by-2 lower right block is $\sigma^{2}$ times invertible. Notice that in this form the 10 and 11 entries vanish to one order higher than before. Then $P(\sigma) \tilde{P}(\sigma)^{-1}$ is invertible for $\sigma \neq 0$, and the inverse has block matrix with bounds

$$
\left(\begin{array}{ccc}
O(1) & O\left(|\sigma|^{-1-\epsilon}\right) & O\left(|\sigma|^{-\epsilon}\right) \\
O\left(|\sigma|^{-\epsilon}\right) & O\left(|\sigma|^{-2}\right) & O\left(|\sigma|^{-2}\right) \\
O\left(|\sigma|^{-\epsilon}\right) & O\left(|\sigma|^{-1}\right) & O\left(|\sigma|^{-2}\right)
\end{array}\right) .
$$

This structure can happen in interesting examples. For instance, for the wave equation on 1-forms on Kerr space, 0-resonances can be read off from [23. Theorem 4.4, Lemma 4.6, Section 5] (while this paper is in the Kerr-de Sitter setting, appropriate explicit resonant and dual resonant states persist in Kerr, with the appropriateness coming from only considering solutions with desired asymptotics as $r \rightarrow \infty)$. In particular, for Schwarzschild there is a resonant state which is a suitable linear combination of $d r$ and $d t$, with non-vanishing $O\left(r^{-1}\right)$ leading term, but the dual state is a delta distribution on the horizon, thus is identically zero near the Euclidean end. Indeed, this delta distributional nature of the dual state persists for slowly rotating Kerr space, see [23. Section 5], so in fact this structure is stable within the family.

\section{REFERENCES}

[1] Lars Andersson and Pieter Blue. Uniform energy bound and asymptotics for the Maxwell field on a slowly rotating Kerr black hole exterior. J. Hyperbolic Differ. Equ., 12(4):689-743, 2015.

[2] Dean Baskin, András Vasy, and Jared Wunsch. Asymptotics of radiation fields in asymptotically Minkowski space. Amer. J. Math., 137(5):1293-1364, 2015.

[3] Jean-François Bony and Dietrich Häfner. Local energy decay for several evolution equations on asymptotically Euclidean manifolds. Ann. Sci. Éc. Norm. Supér. (4), 45(2):311-335, 2012.

[4] Jean-François Bony and Dietrich Häfner. Decay and non-decay of the local energy for the wave equation on the de Sitter-Schwarzschild metric. Comm. Math. Phys., 282(3):697-719, 2008.

[5] Jean-François Bony and Dietrich Häfner. Low frequency resolvent estimates for long range perturbations of the Euclidean Laplacian. Math. Res. Lett., 17(2):301-306, 2010.

[6] Jean-Michel Bony. Calcul symbolique et propagation des singularités pour les équations aux dérivées partielles non linéaires. Ann. Sci. École Norm. Sup. (4), 14(2):209-246, 1981.

[7] Mihalis Dafermos, Gustav Holzegel, and Igor Rodnianski. The linear stability of the Schwarzschild solution to gravitational perturbations. Preprint, arXiv:1601.06467, 2016.

[8] Mihalis Dafermos and Igor Rodnianski. Decay of solutions of the wave equation on Kerr exterior space-times I-II: The cases of $|a| \ll m$ or axisymmetry. Preprint, arXiv:1010.5132, 2010.

[9] Mihalis Dafermos, Igor Rodnianski, and Yakov Shlapentokh-Rothman. Decay for solutions of the wave equation on Kerr exterior spacetimes III: The full subextremal case $|a|<M$. Ann. of Math. (2), 183(3):787-913, 2016.

[10] Maarten de Hoop, Gunther Uhlmann, and András Vasy. Diffraction from conormal singularities. Ann. Sci. Éc. Norm. Supér. (4), 48(2):351-408, 2015.

[11] J. J. Duistermaat. On Carleman estimates for pseudo-differential operators. Invent. Math., 17:31-43, 1972 .

[12] S. Dyatlov and M. Zworski. Mathematical theory of scattering resonances. Preprint, v. 0.1, In preparation. 
[13] Semyon Dyatlov and Maciej Zworski. Dynamical zeta functions for Anosov flows via microlocal analysis. Ann. Sci. Éc. Norm. Supér. (4), 49(3):543-577, 2016.

[14] Frédéric Faure and Johannes Sjöstrand. Upper bound on the density of Ruelle resonances for Anosov flows. Comm. Math. Phys., 308(2):325-364, 2011.

[15] C. Gérard. Semiclassical resolvent estimates for two and three body Schrödinger operators. Comm. P.D.E., 15:1161-1178, 1990.

[16] C. Gérard and A. Martinez. Principe d'absorption limite pour des opérateurs de Schrödinger à longue portées. C.R. Acad. Sci. Paris, 306:121-123, 1988.

[17] Colin Guillarmou and Andrew Hassell. Resolvent at low energy and Riesz transform for Schrödinger operators on asymptotically conic manifolds. I. Math. Ann., 341(4):859-896, 2008.

[18] Colin Guillarmou and Andrew Hassell. Resolvent at low energy and Riesz transform for Schrödinger operators on asymptotically conic manifolds. II. Ann. Inst. Fourier (Grenoble), 59(4):1553-1610, 2009.

[19] V. Guillemin and G. Uhlmann. Oscillatory integrals with singular symbols. Duke Math. J., 48(1):251-267, 1981.

[20] A. Hassell and A. Vasy. Symbolic functional calculus and N-body resolvent estimates. J. Func. Anal., 173:257-283, 2000.

[21] B. Helffer and J. Sjöstrand. Équation de Schrödinger avec champ magnétique et équation de Harper. In Schrödinger operators (Sønderborg, 1988), pages 118-197. Springer, Lecture Notes in Physics, No. 345, Berlin, 1989.

[22] P. Hintz and A. Vasy. The global non-linear stability of the Kerr-de Sitter family of black holes. Acta Math, 220:1-206, 2018.

[23] P. Hintz and A. Vasy. Asymptotics for the wave equation on differential forms on Kerr-de Sitter space. J. Diff. Geo., arXiv:1502.03179, to appear.

[24] Peter Hintz and András Vasy. Semilinear wave equations on asymptotically de Sitter, Kerr-de Sitter and Minkowski spacetimes. Anal. PDE, 8(8):1807-1890, 2015.

[25] Pei-Ken Hung, Jordan Keller, and Mu-Tao Wang. Linear stability of Schwarzschild spacetime: The Cauchy problem of metric coefficients. Preprint, arXiv:1702.02843, 2017.

[26] A. Jensen and T. Kato. Spectral properties of Schrödinger operators and time-decay of the wave functions. Duke Math. J., 46:583-611, 1979.

[27] R. B. Melrose. Spectral and scattering theory for the Laplacian on asymptotically Euclidian spaces. Marcel Dekker, 1994.

[28] R. B. Melrose and G. A. Uhlmann. Lagrangian intersection and the Cauchy problem. Comm. Pure and Appl. Math., 32:483-519, 1979.

[29] Richard B. Melrose. The Atiyah-Patodi-Singer index theorem, volume 4 of Research Notes in Mathematics. A K Peters Ltd., Wellesley, MA, 1993.

[30] Jörn Müller and Alexander Strohmaier. The theory of Hahn-meromorphic functions, a holomorphic Fredholm theorem, and its applications. Anal. PDE, 7(3):745-770, 2014.

[31] Tullio Regge and John A. Wheeler. Stability of a Schwarzschild Singularity. Phys. Rev., 108:1063-1069, Nov 1957.

[32] D. Robert and H. Tamura. Semiclassical estimates for resolvents and asymptotics for total scattering cross-sections. Ann. Inst. H. Poincaré (phys. théor.), 47:415-442, 1987.

[33] Igor Rodnianski and Terence Tao. Effective limiting absorption principles, and applications. Comm. Math. Phys., 333(1):1-95, 2015.

[34] Yakov Shlapentokh-Rothman. Quantitative mode stability for the wave equation on the Kerr spacetime. Ann. Henri Poincaré, 16(1):289-345, 2015.

[35] Johannes Sjöstrand and Maciej Zworski. Complex scaling and the distribution of scattering poles. J. Amer. Math. Soc., 4(4):729-769, 1991.

[36] Johannes Sjöstrand and Maciej Zworski. Fractal upper bounds on the density of semiclassical resonances. Duke Math. J., 137(3):381-459, 2007.

[37] Daniel Tataru. Local decay of waves on asymptotically flat stationary space-times. Amer. J. Math., 135(2):361-401, 2013.

[38] Daniel Tataru and Mihai Tohaneanu. A local energy estimate on Kerr black hole backgrounds. Int. Math. Res. Not. IMRN, (2):248-292, 2011.

[39] André Unterberger. Résolution d'équations aux dérivées partielles dans des espaces de distributions d'ordre de régularité variable. Ann. Inst. Fourier (Grenoble), 21(2):85-128, 1971. 
[40] A. Vasy. Microlocal analysis of asymptotically hyperbolic and Kerr-de Sitter spaces. Inventiones Math., 194:381-513, 2013. With an appendix by S. Dyatlov.

[41] A. Vasy and J. Wunsch. Semiclassical second microlocal propagation of regularity and integrable systems. J. d'Analyse Mathematique, arxiv:math/0801.0826, 108:119-157, 2009.

[42] A. Vasy and J. Wunsch. Positive commutators at the bottom of the spectrum. J. Func. Anal, 259:503-523, 2010.

[43] A. Vasy and M. Zworski. Semiclassical estimates in asymptotically Euclidean scattering. Commun. Math. Phys., 212:205-217, 2000.

[44] András Vasy. On the positivity of propagator differences. Ann. Henri Poincaré, 18(3):9831007, 2017.

[45] András Vasy. A minicourse on microlocal analysis for wave propagation. In Asymptotic analysis in general relativity, volume 443 of London Math. Soc. Lecture Note Ser., pages 219-374. Cambridge Univ. Press, Cambridge, 2018.

[46] X.P. Wang. Time-decay of scattering solutions and classical trajectories. Ann. Inst. H. Poincaré (phys. théor.), 47:25-37, 1987.

[47] Bernard F. Whiting. Mode stability of the Kerr black hole. J. Math. Phys., 30(6):1301-1305, 1989.

Department of Mathematics, Stanford University, CA 94305-2125, USA

E-mail address: andras@math.stanford.edu 